\title{
ANALYSIS OF A NONLINEAR FLUID-STRUCTURE INTERACTION MODEL WITH MECHANICAL DISSIPATION AND DELAY
}

\author{
GILBERT PERALTA* AND KARL KUNISCH ${ }^{\ddagger}$
}

\begin{abstract}
A fluid-structure interaction model with discrete and distributed delays in the structural damping is studied. The fluid and structure dynamics are governed by the Navier-Stokes and linear elasticity equations, respectively. Due to the presence of delay, a crucial ingredient of the weak formulation is the use of hidden boundary regularity for transport equations. In two space dimension, it is shown that weak solutions are unique. For smooth and compatible data, we establish the existence of the pressure and by applying micro-local analysis, further regularity of the solutions are available. Finally, the exponential stability of the system is obtained through an appropriate Lyapunov functional.
\end{abstract}

\section{INTRODUCTION}

In this paper, we study the well-posedness and stability of an incompressible fluidstructure interaction model with interior damping and delay in the structure. The NavierStokes equation will be used to model the behavior of the fluid, while a linear elasticity equation is used for the solid. Continuity of the velocities and the normal components of the stress tensors across the interface is imposed. The interface is assumed to be stationary, a reasonable assumption under the case of small but rapid oscillations of the elastic body. We consider the following nonlinear fluid-structure interaction model with mechanical dissipation and delay

$$
\begin{cases}u_{t}(t, x)-\Delta u(t, x)+u(t, x) \cdot \nabla u(t, x)+\nabla p(t, x)=G_{f}(t, x), & t>0, x \in \Omega_{f}, \\ \operatorname{div} u(t, x)=0, & t>0, x \in \Omega_{f}, \\ u(t, x)=0, & t>0, x \in \Gamma_{f}, \\ u(t, x)=w_{t}(t, x), & t>0, x \in \Gamma_{s}, \\ w_{t t}(t, x)-\operatorname{div} \sigma(w(t, x))+w(t, x)+\mu w_{t}(t, x) & \\ \quad+\mu_{d} w_{t}\left(t-r_{d}, x\right)+\int_{-r_{c}}^{0} \mu_{c}(\theta) w_{t}(t+\theta, x) \mathrm{d} \theta=G_{s}(t, x), & t>0, x \in \Omega_{s}, \\ \frac{\partial}{\partial \nu} u(t, x)-p(t, x) \nu(x)+\frac{1}{2}(u(t, x) \cdot \nu(x)) u(t, x) & \\ \quad=\sigma(w(t, x)) \cdot \nu(x), & t>0, x \in \Gamma_{s},\end{cases}
$$

2010 Mathematics Subject Classification. 74F10, 35Q30, 93D20, 93D15.

Key words and phrases. Fluid-Structure Interaction Model, Delay, Exponential Stability, Lyapunov Functional.

* Department of Mathematics and Computer Science, University of the Philippines Baguio, Governor Pack Road, 2600 Baguio, Philippines. E-mail: grperalta@up.edu.ph

${ }^{\ddagger}$ Institute for Mathematics and Scientific Computing, University of Graz, Heinrichstrasse 36, 8010 Graz, Austria and RICAM Institute, Austrian Academy of Sciences, Altenbergerstrasse 69, 4040 Linz Austria. E-mail: karl.kunisch@uni-graz.at

This work is supported by the ERC advanced grant 668998 (OCLOC) under the EU's H2020 research program. 
where the linearized elastic deformation tensor $\sigma$ is given by $\sigma(w)=2 \mu_{s} \epsilon(w)+\lambda_{s}(\operatorname{div} w) I$, with $\epsilon(w)=\frac{1}{2}\left(\nabla w+\nabla w^{T}\right)$ being the symmetric part of $\nabla w$. The positive constants $\mu_{s}$ and $\lambda_{s}$ depend on the material properties of the solid. Due to incompressibility, the stress tensor for the fluid reduces to the Laplacian.

The vector-valued functions $u:[0, T] \times \Omega_{f} \rightarrow \mathbb{R}^{d}$ and $w:[0, T] \times \Omega_{s} \rightarrow \mathbb{R}^{d}$ represent the fluid velocity and structure displacement, respectively, while the scalar-valued function $p:[0, T] \times \Omega_{f} \rightarrow \mathbb{R}$ denote the fluid pressure. We assume that $\Omega_{f}$ and $\Omega_{s}$ are sufficiently smooth domains in $\mathbb{R}^{d}$ with either $d=2$ or $d=3$. The interface $\Gamma_{s}$ and external fluid boundary $\Gamma_{f}$ have no points in common and are a positive distance apart. Here, $\nu$ will be the unit normal outward to $\Gamma_{s}$ in $\Omega_{s}$ and to $\Gamma_{f}$ in $\Omega_{f}$. The functions $G_{f}$ and $G_{s}$ are external sources in the fluid and structure dynamics.

We denote the respective initial fluid velocity and structure displacement, velocity and history as follows

$$
\left\{\begin{array}{l}
u(0, x)=u_{0}(x), x \in \Omega_{f} \\
w(0, x)=w_{0}(x), x \in \Omega_{s} \\
w_{t}(0, x)=v_{0}(x), x \in \Omega_{s} \\
w_{t}(\theta, x)=z_{0}(\theta, x), \theta \in(-r, 0), x \in \Omega_{s}
\end{array}\right.
$$

where $r=\max \left(r_{d}, r_{c}\right)$. System (1.1) without delay has been extensively studied by several authors, see $[\mathbf{9}, \mathbf{1 0}, \mathbf{1 6}, \mathbf{2 8}]$ for instance. See also $[\mathbf{3}, \mathbf{5}, \mathbf{6}, \mathbf{7}, \mathbf{2 0}, \mathbf{2 1}, \mathbf{2 4}, \mathbf{2 5}, \mathbf{3 0}, \mathbf{3 1}]$ for other related topics.

On the interface, the appearance of the term $\frac{1}{2}(u \cdot \nu) u$ is due to the hypothesis that $\Gamma_{s}$ is stationary. According to the incompressibility of the fluid, it holds that $(u \cdot \nabla) u=\operatorname{div}(u \otimes u)$, and this corresponds to the boundary term $(u \otimes u) \nu=(u \cdot \nu) u$ on $\Gamma_{s}$ when the divergence theorem is applied in the weak formulation. The factor $\frac{1}{2}$ is imposed under the requirement that the energy dissipates due to the fluid diffusion and structural mechanical dissipation. We refer to [9] for other details and relevant references pertaining to the model without delay.

The last three terms on the left hand side of the elasticity equation can be thought of as a feedback control with delay taken into account. This entails that there is a time-lag for the effectivity of the control. Discrete and distributed delays are considered here. The positive constants $r_{d}$ and $r_{c}$ measure the extent of discrete and distributed delays, while $\mu_{d}$ and $\mu_{c}$ are the strengths of such, respectively. We suppose that $\mu_{c}:\left(-r_{c}, 0\right) \rightarrow \mathbb{R}$ is Lebesgue square integrable. We only consider a single discrete delay, nevertheless, the methods presented here can be adapted to the case of multiple discrete delays.

Existence of energy-level weak solutions will be shown by a standard Faedo-Galerkin method using the normalized eigenvectors of the Stokes-Neumann operator. By energylevel weak solutions, we mean that solutions lie on the usual state space formulations for the Navier-Stokes and Lamé's equations. Due to the presence of delay, a crucial ingredient of the weak formulation is the use of hidden boundary regularity for transport equations, see Theorem 3.2 below. In two space dimensions, we will prove that such weak solutions are unique. For smooth and compatible data, we will show the existence of the pressure. By flattening the boundary through Melrose-Sjöstrand coordinates [32, 33], further regularity of the solutions will be proved. This information will be useful in establishing the exponential stability of the system using the energy method. These strategies have been successfully applied to the analysis of fluid-structure interaction models without delay in $[\mathbf{9}, \mathbf{1 0}, \mathbf{1 6}, \mathbf{2 8}]$ and the references therein. 
We also consider the asymptotic behavior of the energy

$$
\begin{aligned}
E(t) & =\frac{1}{2} \int_{\Omega_{f}}|u(t, x)|^{2} \mathrm{~d} x+\frac{1}{2} \int_{\Omega_{s}}\left|w_{t}(t, x)\right|^{2}+|\nabla w(t, x)|^{2} \mathrm{~d} x \\
& +\frac{1}{2} \int_{-r}^{0} \int_{\Omega_{s}}\left|w_{t}(t+\theta, x)\right|^{2} \mathrm{~d} \theta \mathrm{d} x .
\end{aligned}
$$

These quantities represent the kinetic energy of the fluid motion, the total energy of the structural motion and the energy associated with the delay. Without external sources, that is $G_{f}=0$ and $G_{s}=0$, the exponential decay of the energy for the coupled system under suitable assumptions on $\mu, \mu_{d}$ and $\mu_{c}$ will be established using an appropriate Lyapunov functional. For discrete delays, the pioneering works $[\mathbf{1 3}, \mathbf{1 4}]$ are classical references illustrating the destabilizing effect of delays in feedback controls. Typically, if damping dominates the strength of delay then the system is stable, provided that it is stable in the absence of delay.

We reiterate here the challenges when dealing with the fluid-structure model (1.1)-(1.2). First, we have a mismatch of regularity between the dynamics of the fluid (parabolic) and the solid (hyperbolic) on the interface. Second, due to the presence of the Neumann-type boundary condition on the interface, the typical Leray's projection method for the NavierStokes equation is not applicable. Third, the existence of weak solutions for rough initial data with finite energy has to be established. Finally, due to delay, one needs an appropriate functional analytic framework to deal with the retarded terms and to have the existence of weak solutions without any structural assumptions on the coefficient $\mu$, as for instance $\mu \geq \mu_{d}$.

The first three issues have been resolved by Lions [28] and Barbu, Grujić, Lasiecka and Tuffaha [9]. One may overcome the regularity mismatch on the interface by adding small viscoelastic damping on the structure as in $[\mathbf{1 2}, \mathbf{4 5}]$. This turns the coupled parabolichyperbolic system to an entire parabolic problem. On the other hand, local-in-time strong solutions can be achieved with additional regularity and compatibility of the data as in [11]. In establishing the existence of weak solutions, we do not consider smoothing effects on the structural dynamics and we assume that the initial data are not smooth. In this direction, we closely follow the frameworks in [9] and [28].

For the last concern, we follow the paper of Nicaise and Pignotti [36] for multidimensional linear wave equations with boundary or interior damping and delay. The linearized case, with the elasticity equation replaced by the wave equation and neglecting transversal elastic forces, has been studied in [38] using semigroup theory and classical Tauberian theorems. Well-posedness for every constant delay factors can be achieved from the perturbation theorem for strongly continuous semigroups.

With regards to wave equations with distributed delays, [37] considered a delay variable which satisfies a transport equation with two extra parameters and a degenerate kernel. The well-posedness of the model has been established via semigroup methods. A weighted $L^{2}$-space has been considered as the state space for the delay variable, where the weight function is related to the kernel corresponding to the distributed delay. Consequently, it is not necessarily the usual $L^{2}$-space. However, the natural set-up would be the $L^{2}$-space as in the case of discrete delays and for ordinary delay differential equations. This will be the direction we pursue here.

Introducing a new state variable $z(t, \theta, x)=w_{t}(t+\theta, x)$ for $(t, \theta, x) \in(0, \infty) \times(-r, 0) \times \Omega_{s}$ that keeps track of the delay, the elasticity equation can be rewritten as a coupled system 
of hyperbolic partial differential equations

$$
\left\{\begin{array}{l}
z_{t}(t, \theta, x)-z_{\theta}(t, \theta, x)=0 \\
z(t, 0, x)=w_{t}(t, x) \\
w_{t t}(t, x)-\operatorname{div} \sigma(w(t, x))+w(t, x)+\mu w_{t}(t, x) \\
\quad+\mu_{d} z\left(t,-r_{d}, x\right)+\int_{-r_{c}}^{0} \mu_{c}(\theta) z(t, \theta, x) \mathrm{d} \theta=G_{s}(t, x),
\end{array}\right.
$$

for $t>0,-r<\theta<0$ and $x \in \Omega_{s}$. Observe that the first equation in (1.3) is a onedimensional transport equation with parameter $x \in \Omega_{s}$.

The paper is organized as follows. Section 2 deals with the functional analytic properties of the Stokes-Neumann operator. As a strictly positive self-adjoint operator with compact inverse, this map has an orthonormal basis, and this will be used in the Faedo-Galerkin approximations of (1.1). The well-posedness as well as boundary and interior-point trace regularities of the transport equation with a parameter will be briefly presented in Section 3. Existence of energy-level weak solutions of (1.1) will be discussed in Section 4. In the two-dimensional case, the uniqueness of weak solutions, regularity of weak solutions and exponential decay of the energy will be tackled in Sections 5, 6, and 7, respectively.

\section{The Stokes-Neumann Map}

Given an open set $\Omega$ in $\mathbb{R}^{d}$, the inner product on the Lebesgue space $L^{2}(\Omega)$ will be denoted by $(\cdot, \cdot)_{\Omega}$. The usual notation $H^{s}(\Omega)$ with $s \in \mathbb{R}$ for the Sobolev spaces will be used. For simplicity, the product of $d$ copies of a Banach space $X$ will be denoted again by $X$ instead of $X^{d}$. We use the notation $X^{\prime}$ for the dual of $X$, that is, the space of all bounded linear functionals on $X$. The set of all vector-valued functions in $C_{0}^{\infty}(\Omega)$ that are divergence free in $\Omega$ will be denoted by $C_{0, \sigma}^{\infty}(\Omega)$.

We denote the whole fluid-structure domain by $\Omega=\Omega_{f} \cup \Omega_{s} \cup \Gamma_{s}$. In the weak formulation of the Navier-Stokes equation, the standard function spaces of solenoidal functions will be utilized here. Consider the following Hilbert spaces

$$
\begin{aligned}
& H_{f}=\left\{u \in L^{2}\left(\Omega_{f}\right): \operatorname{div} u=0 \text { in } \Omega_{f}, u \cdot \nu=0 \text { on } \Gamma_{f}\right\}, \\
& V_{f}=\left\{u \in H^{1}\left(\Omega_{f}\right): \operatorname{div} u=0 \text { in } \Omega_{f}, u=0 \text { on } \Gamma_{f}\right\},
\end{aligned}
$$

endowed respectively with the following inner products

$$
(u, v)_{\Omega_{f}}=\int_{\Omega_{f}} u \cdot v \mathrm{~d} x, \quad(u, v)_{V_{f}}=\int_{\Omega_{f}} \nabla u: \nabla v \mathrm{~d} x
$$

where the colon represents the inner product in $\mathbb{R}^{d \times d}$. The corresponding norms will be denoted by $\|\cdot\|_{\Omega_{f}}$ and $\|\cdot\|_{V_{f}}$, respectively. Recall that the gradient of a vector field $u=\left(u_{1}, \ldots, u_{d}\right)$ is given by $\nabla u=\left(\partial_{j} u_{k}\right)_{j, k=1}^{d}$. If $u \in H_{f}$ then $\langle u \cdot \nu, 1\rangle_{H^{-1 / 2}\left(\Gamma_{s}\right) \times H^{1 / 2}\left(\Gamma_{s}\right)}=0$ and if $u \in V_{f}$ then $(u, \nu)_{\Gamma_{s}}=0$ from the divergence theorem.

The embedding $V_{f} \subset H_{f}$ is continuous, dense and compact. One can see that the completions of the set $D\left(\Omega_{f}\right)$ consisting of all vector-valued functions in $C^{\infty}\left(\bar{\Omega}_{f}\right)$ which are divergence free in $\Omega_{f}$ and vanish on a neighborhood of $\Gamma_{f}$ under the norms in $L^{2}\left(\Omega_{f}\right)$ and $H_{0}^{1}\left(\Omega_{f}\right)$ are $H_{f}$ and $V_{f}$, respectively. These facts are well-known, however, for completeness we present the details regarding the completions based on appropriate liftings on the interface $\Gamma_{s}$.

Given $u \in H_{f}$, define $z=\left(\nabla z_{f}\right) \chi_{\Omega_{f}}+\left(\nabla z_{s}\right) \chi_{\Omega_{s}} \in L^{2}(\Omega)$, where $\chi$ denotes the characteristic function, and $z_{f} \in H^{1}\left(\Omega_{f}\right)$ and $z_{s} \in H^{1}\left(\Omega_{s}\right)$ are weak solutions, which are unique 
up to additive constants, of the elliptic Neumann boundary value problems

$$
\left\{\begin{array} { l l } 
{ - \Delta z _ { f } = 0 , } & { \text { in } \Omega _ { f } , } \\
{ \frac { \partial z _ { f } } { \partial \nu } = 0 , } & { \text { on } \Gamma _ { f } , } \\
{ \frac { \partial z _ { f } } { \partial \nu } = u \cdot \nu , } & { \text { on } \Gamma _ { s } , }
\end{array} \quad \text { and } \quad \left\{\begin{array}{ll}
-\Delta z_{s}=0, & \text { in } \Omega_{s} \\
\frac{\partial z_{s}}{\partial \nu}=u \cdot \nu, & \text { on } \Gamma_{s} .
\end{array}\right.\right.
$$

Due to the compatibility condition $\langle u \cdot \nu, 1\rangle_{H^{-1 / 2}\left(\Gamma_{s}\right) \times H^{1 / 2}\left(\Gamma_{s}\right)}=0$, the existence of solutions is guaranteed, see [29]. By construction, it holds that $\operatorname{div} z=0$ in $\Omega$ in the sense of distributions and $z \cdot \nu=0$ on $\partial \Omega=\Gamma_{f}$. Therefore, given $\varepsilon>0$, there exists $\varphi \in C_{0, \sigma}^{\infty}(\Omega)$ such that $\|z-\varphi\|_{\Omega}<\varepsilon / 2$. On the other hand, since $u-z$ is divergence free in $\Omega_{f}$ and $(u-z) \cdot \nu=0$ on $\partial \Omega_{f}=\Gamma_{f} \cup \Gamma_{s}$, we have $\|(u-z)-\psi\|_{\Omega_{f}}<\varepsilon / 2$ for some $\psi \in C_{0, \sigma}^{\infty}\left(\Omega_{f}\right)$. Letting $\phi=\varphi+\psi$, we have $\phi \in D\left(\Omega_{f}\right)$ and $\|u-\phi\|_{\Omega_{f}}<\varepsilon$, proving the density of $D\left(\Omega_{f}\right)$ in $H_{f}$.

Now suppose that $u \in V_{f}$ and let $y=y_{f} \chi_{\Omega_{f}}+y_{s} \chi_{\Omega_{s}} \in H_{0}^{1}(\Omega)$, where $\left(y_{f}, \pi_{f}\right) \in$ $H^{1}\left(\Omega_{f}\right) \times\left(L^{2}\left(\Omega_{f}\right) / \mathbb{R}\right)$ and $\left(y_{s}, \pi_{s}\right) \in H^{1}\left(\Omega_{s}\right) \times\left(L^{2}\left(\Omega_{s}\right) / \mathbb{R}\right)$ are the weak solutions of the following Stokes problems

$$
\left\{\begin{array} { l l } 
{ - \Delta y _ { f } + \nabla \pi _ { f } = 0 , } & { \text { in } \Omega _ { f } , } \\
{ \operatorname { d i v } y _ { f } = 0 , } & { \text { in } \Omega _ { f } , } \\
{ y _ { f } = 0 , } & { \text { on } \Gamma _ { f } , } \\
{ y _ { f } = u , } & { \text { on } \Gamma _ { s } , }
\end{array} \quad \text { and } \quad \left\{\begin{array}{ll}
-\Delta y_{s}+\nabla \pi_{s}=0, & \text { in } \Omega_{s}, \\
\operatorname{div} y_{s}=0, & \text { in } \Omega_{s}, \\
y_{s}=u, & \text { on } \Gamma_{s} .
\end{array}\right.\right.
$$

Thanks to the compatibility condition $(u, \nu)_{\Gamma_{s}}=0$, these problems are well-posed, see [43] for instance. According to the definition, div $y=0$ in $\Omega$ and therefore given $\varepsilon>0$ we have $\|\nabla y-\nabla \varphi\|_{\Omega}<\varepsilon / 2$ for some $\varphi \in C_{0, \sigma}^{\infty}(\Omega)$. Similarly, there exists $\psi \in C_{0, \sigma}^{\infty}\left(\Omega_{f}\right)$ satisfying $\|\nabla(u-y)-\nabla \psi\|_{\Omega_{f}}<\varepsilon / 2$ because $u-y \in H_{0}^{1}\left(\Omega_{f}\right)$ and $\operatorname{div}(u-y)=0$ in $\Omega_{f}$. Consequently, if $\phi=\varphi+\psi \in D\left(\Omega_{f}\right)$ then $\|\nabla u-\nabla \phi\|_{\Omega_{f}}<\varepsilon$, showing the density of $D\left(\Omega_{f}\right)$ in $V_{f}$.

We also consider the Hilbert spaces $H_{0}=H_{f} \times L^{2}\left(\Omega_{s}\right)$ and $H_{1}=\left\{(u, v) \in V_{f} \times H^{1}\left(\Omega_{s}\right)\right.$ : $u=v$ on $\left.\Gamma_{s}\right\}$ with the inner products

$$
\begin{aligned}
& ((u, v),(\varphi, \psi))_{H_{0}}=\int_{\Omega_{f}} u \cdot \varphi \mathrm{d} x+\int_{\Omega_{s}} v \cdot \psi \mathrm{d} x, \\
& ((u, v),(\varphi, \psi))_{H_{1}}=\int_{\Omega_{f}} \nabla u: \nabla \varphi \mathrm{d} x+\int_{\Omega_{s}} \nabla v: \nabla \psi \mathrm{d} x .
\end{aligned}
$$

In $H_{1}$, the norm $\|\cdot\|_{H_{1}}$ is equivalent to

$$
\|(u, v)\|=\left(\int_{\Omega_{f}}|\nabla u|^{2} \mathrm{~d} x+\int_{\Omega_{s}}|v|^{2}+|\nabla v|^{2} \mathrm{~d} x\right)^{\frac{1}{2}} .
$$

Indeed, if $(u, v) \in H_{1}$ then $\xi=u \chi_{\Omega_{f}}+v \chi_{\Omega_{s}} \in H_{0}^{1}(\Omega)$. By the Poincaré inequality

$$
\|(u, v)\|^{2} \leq \int_{\Omega}|\nabla \xi|^{2}+|\xi|^{2} \mathrm{~d} x \leq c_{\Omega} \int_{\Omega}|\nabla \xi|^{2} \mathrm{~d} x=c_{\Omega}\|(u, v)\|_{H_{1}}^{2},
$$

for some $c_{\Omega}>0$. The other inequality is trivial. We have the following density property.

Proposition 2.1. The set $D:=\left\{(u, v) \in D\left(\Omega_{f}\right) \times C^{\infty}\left(\bar{\Omega}_{s}\right): u \chi_{\Omega_{f}}+v \chi_{\bar{\Omega}_{s}} \in C_{0}^{\infty}(\Omega)\right\}$ is dense in $H_{0}$ and $H_{1}$ with respect to their underlying norms. 
Proof. Denote by $\tilde{H}_{0}$ and $\tilde{H}_{1}$ the closures of $D$ in $H_{0}$ and $H_{1}$, respectively. It is clear that $\tilde{H}_{i} \subset H_{i}$ for $i=0,1$. Let $(u, v) \in H_{0}$ and suppose that $((u, v),(\varphi, \psi))_{H_{0}}=0$ for every $(\varphi, \psi) \in D$. By extending every $\psi \in C_{0}^{\infty}\left(\Omega_{s}\right)$ by zero outside $\Omega_{s}$, we have $(v, \psi)_{\Omega_{s}}=0$, hence $v=0$. Consequently, $u=0$ as well by the density of $D\left(\Omega_{f}\right)$ in $H_{f}$. Thus, $\tilde{H}_{0}=H_{0}$.

To prove that $\tilde{H}_{1}=H_{1}$, we follow $[\mathbf{4 3}$, Section I.1] by showing that any bounded linear functional $L$ on $H_{1}$ that vanishes on $\tilde{H}_{1}$ is identically zero. Let us define a bounded linear functional $\tilde{L}$ on $W:=\left\{\xi \in H_{0}^{1}(\Omega): \operatorname{div} \xi=0\right.$ in $\left.\Omega_{f}\right\}$, considered as a subspace of $H_{0}^{1}(\Omega)$, by

$$
\langle\tilde{L}, \xi\rangle_{W^{\prime} \times W}=\left\langle L,\left(\left.\xi\right|_{\Omega_{f}},\left.\xi\right|_{\Omega_{s}}\right)\right\rangle_{H_{1}^{\prime} \times H_{1}} .
$$

This functional is well-defined since the map $\xi \mapsto\left(\left.\xi\right|_{\Omega_{f}},\left.\xi\right|_{\Omega_{s}}\right)$ is an isometric isomorphism from $W$ onto $H_{1}$, and moreover, it vanishes on $\left\{\xi \in C_{0}^{\infty}(\Omega): \xi \in D\left(\Omega_{f}\right)\right\}$. By the HahnBanach Theorem, $\tilde{L}$ can be extended to an element of $H^{-1}(\Omega)$. Now, $\tilde{L}$ vanishes on $C_{0, \sigma}^{\infty}(\Omega)$, and therefore $\tilde{L}=\nabla p$ for some $p \in L^{2}(\Omega)$ in the distributional sense. This implies that

$$
\langle\tilde{L}, \xi\rangle_{W^{\prime} \times W}=-(p, \operatorname{div} \xi)_{\Omega_{s}}, \quad \text { for all } \xi \in W .
$$

Taking arbitrary $\xi \in C_{0}^{\infty}\left(\Omega_{s}\right)$, extending it by zero outside $\Omega_{s}$ and then using $\langle\tilde{L}, \xi\rangle_{W^{\prime} \times W}=$ 0 , we have $\nabla p=0$ in $\Omega_{s}$. Thus, $p$ is constant in $\Omega_{s}$ and we obtain from the divergence theorem that

$$
\langle\tilde{L}, \xi\rangle_{W^{\prime} \times W}=-p \int_{\Gamma_{s}} \xi \cdot \nu \mathrm{d} x=p \int_{\Omega_{f}} \operatorname{div} \xi \mathrm{d} x=0
$$

for every $\xi \in W$. Therefore, $\tilde{L}=0$ and as a result $L=0$.

From the above proposition, $H_{0} \subset H_{1}$ is continuous and dense. Moreover, since $L^{2}\left(\Omega_{s}\right) \subset$ $H^{1}\left(\Omega_{s}\right)$ and $H_{f} \subset V_{f}$ are compact embeddings, $H_{1} \subset H_{0}$ is also a compact embedding.

We now define the Stokes-Neumann operator. Roughly speaking, this is a linear operator describing a simplified steady state version of the fluid-structure interaction problem. Let $A: D(A) \subset H_{0} \rightarrow H_{0}$ be the linear operator defined by

$$
A(u, v)=(-\Delta u+\nabla p,-\Delta v)
$$

with domain $D(A)$ consisting of all elements in $(u, v) \in H_{1}$ such that $\Delta v \in L^{2}\left(\Omega_{s}\right),-\Delta u+$ $\nabla p \in H_{f}$ and $\frac{\partial v}{\partial \nu}=\frac{\partial u}{\partial \nu}-p \nu$ in $H^{-\frac{1}{2}}\left(\Gamma_{s}\right)$ for some $p \in L^{2}\left(\Omega_{f}\right)$.

Note that $v \in H^{1}\left(\Omega_{s}\right)$ and $\Delta v \in L^{2}\left(\Omega_{s}\right)$ imply that $\frac{\partial v}{\partial \nu} \in H^{-\frac{1}{2}}\left(\Gamma_{s}\right)$. The fact that $\left.\frac{\partial u}{\partial \nu}\right|_{\Gamma_{s}} \in H^{-\frac{1}{2}}\left(\Gamma_{s}\right)$ and $\left.p\right|_{\Gamma_{s}} \in H^{-\frac{1}{2}}\left(\Gamma_{s}\right)$ follow from $-\Delta u+\nabla p \in H_{f}$ and $u \in V_{f}$, see [5]. By choosing $p=0$, we can see that $D \subset D(A)$, and thus, $D(A)$ is dense in $H_{0}$ by Proposition 2.1. For the Stokes operator with Neumann boundary conditions and without the coupling with the Neumann Laplacian, we refer to [34].

Theorem 2.2. The linear operator $A$ is self-adjoint and strictly positive. Moreover, $D\left(A^{\frac{1}{2}}\right)=$ $H_{1}$.

Proof. We begin by showing that $A$ is symmetric. For this purpose, let $(u, v),(\varphi, \psi) \in D(A)$ and denote by $p$ and $\pi$ be the corresponding elements in $L^{2}\left(\Omega_{f}\right)$. Applying Green's identity 
and the divergence theorem twice, we have

$$
\begin{aligned}
(A(u, v),(\varphi, \psi))_{H_{0}} & =\int_{\Omega_{f}}(-\Delta u+\nabla p) \cdot \varphi \mathrm{d} x+\int_{\Omega_{s}}(-\Delta v) \cdot \psi \mathrm{d} x \\
& =\int_{\Omega_{f}} \nabla u: \nabla \varphi \mathrm{d} x+\int_{\Omega_{s}} \nabla v: \nabla \psi \mathrm{d} x \\
& =\int_{\Omega_{f}} u \cdot(-\Delta \varphi+\nabla \pi) \mathrm{d} x+\int_{\Omega_{s}} v \cdot(-\Delta \psi) \mathrm{d} x \\
& =((u, v), A(\varphi, \psi))_{H_{0}} .
\end{aligned}
$$

Here, we used the boundary conditions $\frac{\partial v}{\partial \nu}=\frac{\partial u}{\partial \nu}-p \nu$ and $\frac{\partial \psi}{\partial \nu}=\frac{\partial \varphi}{\partial \nu}-\pi \nu$ on $\Gamma_{s}, u=\varphi=0$ on $\Gamma_{f}$, and the fact that $u$ and $\varphi$ are divergence free in $\Omega_{f}$.

To prove that $A$ is self-adjoint, it is enough to prove that $A$ is onto according to $[44$, Proposition 3.2.4]. Let $(f, g) \in H_{0}$ be given. Note that the map $(f, g) \mapsto(f, \varphi)_{\Omega_{f}}+$ $(g, \psi)_{\Omega_{s}}$ defines a bounded linear functional on $H_{1}$, and hence from the Riesz representation theorem, there exists a unique $(u, v) \in H_{1}$ such that

$$
(\nabla u, \nabla \varphi)_{\Omega_{f}}+(\nabla v, \nabla \psi)_{\Omega_{s}}=(f, \varphi)_{\Omega_{f}}+(g, \psi)_{\Omega_{s}}, \quad \forall(\varphi, \psi) \in H_{1} .
$$

Taking $\psi=0$ and $\varphi \in H_{0}^{1}\left(\Omega_{f}\right) \cap V_{f}$ in (2.1), it follows from de Rham's theorem that there exists $\tilde{p} \in L^{2}\left(\Omega_{f}\right)$ such that $-\Delta u+\nabla \tilde{p}=f$ in the sense of distributions. Note that $p=\tilde{p}+p^{*}$ for every constant $p^{*}$ satisfies the equation $-\Delta u+\nabla p=f$ in the sense of distributions as well. This constant will be appropriately chosen below. For every $\varphi \in V_{f}$ we have, according to generalized Green's identity,

$$
\int_{\Omega_{f}} f \cdot \varphi \mathrm{d} x=\left\langle\frac{\partial u}{\partial \nu}-p \nu, \varphi\right\rangle_{H^{-\frac{1}{2}}\left(\Gamma_{s}\right) \times H^{\frac{1}{2}}\left(\Gamma_{s}\right)}+\int_{\Omega_{f}} \nabla u: \nabla \varphi \mathrm{d} x .
$$

By taking $\varphi=0$ and $\psi \in H_{0}^{1}\left(\Omega_{s}\right)$ in $(2.1)$ we have $-\Delta v=g$ in $L^{2}\left(\Omega_{s}\right)$. Thus $\frac{\partial v}{\partial \nu} \in$ $H^{-\frac{1}{2}}\left(\Gamma_{s}\right)$ and for every $\psi \in H^{1}\left(\Omega_{s}\right)$ there holds

$$
\int_{\Omega_{s}} g \cdot \psi \mathrm{d} x=-\left\langle\frac{\partial v}{\partial \nu}, \psi\right\rangle_{H^{-\frac{1}{2}}\left(\Gamma_{s}\right) \times H^{\frac{1}{2}}\left(\Gamma_{s}\right)}+\int_{\Omega_{s}} \nabla v: \nabla \psi \mathrm{d} x .
$$

Let $\phi \in H^{\frac{1}{2}}\left(\Gamma_{s}\right)$ and set $\zeta=\phi-\left(\frac{1}{\left|\Gamma_{s}\right|} \int_{\Gamma_{s}} \phi \cdot \nu \mathrm{d} x\right) \nu$. Choose the constant $p^{*}$ according to

$$
p^{*}=\frac{1}{\left|\Gamma_{s}\right|}\left\langle\frac{\partial u}{\partial \nu}-\tilde{p} \nu-\frac{\partial v}{\partial \nu}, \nu\right\rangle_{H^{-\frac{1}{2}}\left(\Gamma_{s}\right) \times H^{\frac{1}{2}}\left(\Gamma_{s}\right)} .
$$

Note that $\zeta \in H^{\frac{1}{2}}\left(\Gamma_{s}\right)$ and $\int_{\Gamma_{s}} \zeta \cdot \nu \mathrm{d} x=0$. From the trace theorem there exists $\psi \in H^{1}\left(\Omega_{s}\right)$ such that $\left.\psi\right|_{\Gamma_{s}}=\zeta$. Let $(\varphi, \pi) \in V_{f} \times L^{2}\left(\Omega_{f}\right) / \mathbb{R}$ be the solution of the following Stokes problem

$$
\begin{cases}-\Delta \varphi+\nabla \pi=0, & \text { in } \Omega_{f}, \\ \operatorname{div} \varphi=0, & \text { in } \Omega_{f}, \\ \varphi=0, & \text { on } \Gamma_{f}, \\ \varphi=\zeta, & \text { on } \Gamma_{s} .\end{cases}
$$

Using $(\varphi, \psi) \in H_{1}$ as the test function in (2.2) and (2.3) we have

$$
\left\langle\frac{\partial u}{\partial \nu}-p \nu-\frac{\partial v}{\partial \nu}, \zeta\right\rangle_{H^{-\frac{1}{2}}\left(\Gamma_{s}\right) \times H^{\frac{1}{2}}\left(\Gamma_{s}\right)}=0 .
$$


From the definition of $p^{*}$ and the equation $\phi=\zeta+\left(\frac{1}{\left|\Gamma_{s}\right|} \int_{\Gamma_{s}} \phi \cdot \nu \mathrm{d} x\right) \nu$ we obtain that $\frac{\partial u}{\partial \nu}-p \nu-\frac{\partial v}{\partial \nu}=0$ in $H^{-\frac{1}{2}}\left(\Gamma_{s}\right)$. Therefore, $(u, v) \in D(A)$ and $A(u, v)=(f, g)$.

From the proof of symmetry, we deduce that for every $(u, v) \in D(A)$

$$
(A(u, v),(u, v))_{H_{0}}=\|(u, v)\|_{H_{1}}^{2} .
$$

Using this and the Poincaré inequality, we conclude that $A$ is strictly positive. Finally, we prove that $D\left(A^{\frac{1}{2}}\right)=H_{1}$. By $\left[\mathbf{4 4}\right.$, Remark 3.4.4], the space $D\left(A^{\frac{1}{2}}\right)$ can be viewed as the completion of $D(A)$ with respect to the norm

$$
\|(u, v)\|_{1 / 2}:=(A(u, v),(u, v))_{H_{0}}^{1 / 2},
$$

and therefore from (2.6), we have $D\left(A^{\frac{1}{2}}\right) \subset H_{1}$ since $D(A) \subset H_{1}$. On the other hand, from $D \subset D(A)$ and the density of $D$ in $H_{1}$, we have the reverse inclusion $H_{1} \subset D\left(A^{\frac{1}{2}}\right)$.

The operator $A^{-1}$ is also strictly positive, self-adjoint and compact, the latter being a consequence of the compactness of $H_{1} \subset H_{0}$. From the above discussions and [44, Theorem 12.2.11], $H_{0}$ has an orthonormal basis $\left\{\left(\varphi_{n}, \psi_{n}\right)\right\}_{n=1}^{\infty}$ consisting of normalized eigenvectors of $A$. Let $\lambda_{n}$ be the eigenvalue associated with $\left(\varphi_{n}, \psi_{n}\right)$. Each $\lambda_{n}$ is positive, and we list them in increasing order so that $\lambda_{n} \rightarrow \infty$. Every element $(u, v) \in H_{0}$ has the Fourier series expansion

$$
(u, v)=\sum_{n=1}^{\infty}\left\{\left(u, \varphi_{n}\right)_{\Omega_{f}}+\left(v, \psi_{n}\right)_{\Omega_{s}}\right\}\left(\varphi_{n}, \psi_{n}\right) .
$$

In particular, for $u \in H_{f}$ and $v \in L^{2}\left(\Omega_{s}\right)$ we have

$$
u=\sum_{n=1}^{\infty}\left(u, \varphi_{n}\right)_{\Omega_{f}} \varphi_{n}, \quad v=\sum_{n=1}^{\infty}\left(v, \psi_{n}\right)_{\Omega_{s}} \psi_{n} .
$$

Consequently, the operator $A$ can be expressed as a Fourier series in terms of the associated orthonormal basis as

$$
A(u, v)=\sum_{n=1}^{\infty} \lambda_{n}\left\{\left(u, \varphi_{n}\right)_{\Omega_{f}}+\left(v, \psi_{n}\right)_{\Omega_{s}}\right\}\left(\varphi_{n}, \psi_{n}\right)
$$

and its domain can be alternatively characterized according to

$$
D(A)=\left\{(u, v) \in H_{0}: \sum_{n=1}^{\infty}\left(1+\lambda_{n}^{2}\right)\left|\left(u, \varphi_{n}\right)_{\Omega_{f}}+\left(v, \psi_{n}\right)_{\Omega_{s}}\right|^{2}<\infty\right\} .
$$

In proving the existence of weak solutions to (1.1), we shall utilize the orthogonal projection $\Pi_{m}$ of $H_{0}$ onto the span of $\left\{\left(\varphi_{j}, \psi_{j}\right)\right\}_{j=1}^{m}$ given by

$$
\Pi_{m}(u, v)=\sum_{n=1}^{m}\left\{\left(u, \varphi_{n}\right)_{\Omega_{f}}+\left(v, \psi_{n}\right)_{\Omega_{s}}\right\}\left(\varphi_{n}, \psi_{n}\right) .
$$

For each $(u, v) \in H_{0}$ we have $\left\|\Pi_{m}(u, v)\right\|_{H_{0}} \leq\|(u, v)\|_{H_{0}}$ for all $m$ and $\Pi_{m}(u, v) \rightarrow(u, v)$ as $m \rightarrow \infty$ in $H_{0}$.

Consequently, we have the following characterization

$$
H_{1}=\left\{(u, v) \in H_{0}: \sum_{n=1}^{\infty} \lambda_{n}\left|\left(u, \varphi_{n}\right)_{\Omega_{f}}+\left(v, \psi_{n}\right)_{\Omega_{s}}\right|^{2}<\infty\right\}
$$


and

$$
\|(u, v)\|_{H_{1}}=\left(\sum_{n=1}^{\infty} \lambda_{n}\left|\left(u, \varphi_{n}\right)_{\Omega_{f}}+\left(v, \psi_{n}\right)_{\Omega_{s}}\right|^{2}\right)^{\frac{1}{2}} .
$$

Using this, we have $\left\|\Pi_{m}(u, v)\right\|_{H_{1}} \leq\|(u, v)\|_{H_{1}}$ for each $m$ and $\Pi_{m}(u, v) \rightarrow(u, v)$ as $m \rightarrow \infty$ in $H_{1}$ whenever $(u, v) \in H_{1}$. Indeed,

$$
\left\|\Pi_{m}(u, v)\right\|_{H_{1}}^{2}=\sum_{n=1}^{m} \lambda_{n}\left|\left(u, \varphi_{n}\right)_{\Omega_{f}}+\left(v, \psi_{n}\right)_{\Omega_{s}}\right|^{2} \leq\|(u, v)\|_{H_{1}}^{2}
$$

and as $m \rightarrow \infty$

$$
\left\|\Pi_{m}(u, v)-(u, v)\right\|_{H_{1}}^{2} \leq \sum_{n=m+1}^{\infty} \lambda_{n}\left|\left(u, \varphi_{n}\right)_{\Omega_{f}}+\left(v, \psi_{n}\right)_{\Omega_{s}}\right|^{2} \rightarrow 0 .
$$

\section{Transport Equations with Parameter}

Let $\Omega \subset \mathbb{R}^{d}$ be Lipschitz, $T>0$ and $a<b$. The results of this section will be applied to the case $(a, b)=(-r, 0)$. Consider the transport equation with extra variable $x \in \Omega$

$$
\begin{cases}z_{t}(t, \theta, x)-z_{\theta}(t, \theta, x)=0, & \text { in }(0, T) \times(a, b) \times \Omega, \\ z(t, b, x)=v(t, x), & \text { in }(0, T) \times \Omega, \\ z(0, \theta, x)=z_{0}(\theta, x), & \text { in }(a, b) \times \Omega .\end{cases}
$$

If the initial and boundary data $v$ and $z_{0}$ are sufficiently smooth and satisfy appropriate compatibility conditions, then one can obtain the solution of (3.1) using the method of characteristics. Moreover, one obtains a well-defined trace on the boundary as well at interior points. Our goal is to have weak solutions for square integrable data and recover also this trace regularity. This will be needed in the weak formulation of our fluid-structure interaction model (1.1).

Our approach follows a variational method similar to the method of Friedrichs [17] for symmetric hyperbolic systems. Here, we focus in the bounded case and also mention the construction of traces for the graph spaces associated with (3.1). First, we write our notion of weak solution.

Definition 3.1. Let $v \in L^{2}((0, T) \times \Omega)$ and $z_{0} \in L^{2}((a, b) \times \Omega)$. A function $z \in L^{2}((0, T) \times$ $(a, b) \times \Omega)$ is called a weak solution of $(3.1)$ if the variational equation

$$
-\int_{0}^{T} \int_{a}^{b} \int_{\Omega} z \cdot\left(\phi_{t}-\phi_{\theta}\right) \mathrm{d} x \mathrm{~d} \theta \mathrm{d} t=\left.\int_{a}^{b} \int_{\Omega} z_{0} \cdot \phi\right|_{t=0} \mathrm{~d} x \mathrm{~d} \theta+\left.\int_{0}^{T} \int_{\Omega} v \cdot \phi\right|_{\theta=b} \mathrm{~d} x \mathrm{~d} t
$$

holds for every $\phi \in H^{1}\left((0, T) \times(a, b) ; L^{2}(\Omega)\right)$ such that $\left.\phi\right|_{t=T}=0$ and $\left.\phi\right|_{\theta=a}=0$.

Notice that a weak solution satisfies the equation $z_{t}-z_{\theta}=0$ in the sense of distributions. Hence, one can have a generalized trace on the boundary of $Q_{T}=(0, T) \times(a, b)$. Following $[\mathbf{2}, \mathbf{2 2}, \mathbf{3 9}]$, we shall construct this generalized trace and its restrictions in the Appendix. The proofs of the following theorems are similar to that of hyperbolic systems and for this reason we do not include them here. For the definition of the space $V_{L}^{2}\left(Q_{T}\right)$ we refer to the Appendix.

Theorem 3.2. Given $v \in L^{2}((0, T) \times \Omega)$ and $z_{0} \in L^{2}((a, b) \times \Omega)$, (3.1) has a unique weak solution. Moreover, we have $z \in C\left(0, T ; L^{2}((a, b) \times \Omega)\right) \cap L^{2}\left(\Omega ; V_{L}^{2}\left(Q_{T}\right)\right),\left.z\right|_{\theta=a},\left.z\right|_{\theta=b} \in$ 
$L^{2}((0, T) \times \Omega)$ and for each $\gamma \in \mathbb{R}$ we have

$$
\begin{aligned}
& e^{-2 \gamma t}\|z(t)\|_{L^{2}((a, b) \times \Omega)}^{2}+\left\|\left.e^{-\gamma t} z\right|_{\theta=-r}\right\|_{L^{2}((0, T) \times \Omega)}^{2} \\
& +2 \gamma \int_{0}^{t} e^{-2 \gamma \sigma}\|z(\sigma)\|_{L^{2}((a, b) \times \Omega)}^{2} \mathrm{~d} \sigma=\left\|z_{0}\right\|_{L^{2}((a, b) \times \Omega)}^{2}+\left\|e^{-\gamma t} v\right\|_{L^{2}((0, T) \times \Omega)}^{2}
\end{aligned}
$$

for every $t \in[0, T]$.

The parameter $\gamma$ in the above theorem will be useful in the derivation of a priori estimates applicable to arbitrary delay coefficients $\mu, \mu_{d} \in \mathbb{R}^{d}$ and $\mu_{c} \in L^{2}\left(-r_{c}, 0\right)$.

Theorem 3.3. Suppose that $m, s \geq 0$ are nonnegative integers, $z_{0} \in H^{m}\left((a, b) ; H^{s}(\Omega)\right)$ and $v \in H^{m}\left(0, T ; H^{s}(\Omega)\right)$ satisfy the compatibility conditions $\left.\partial_{t}^{j} v\right|_{t=0}=\left.\partial_{\theta}^{j} z_{0}\right|_{\theta=b}$ for every $j=0,1, \ldots, m-1$. Then the weak solution of (3.1) satisfies

$$
\begin{aligned}
& z \in \bigcap_{j=0}^{m} C^{j}\left(0, T ; H^{m-j}\left((a, b) ; H^{s}(\Omega)\right)\right), \\
& \left.z\right|_{\theta=a},\left.z\right|_{\theta=b} \in H^{m}\left(0, T ; H^{s}(\Omega)\right) .
\end{aligned}
$$

Remark 3.4. Let $\xi \in[a, b]$. By choosing test functions $\phi \in H^{1}\left((0, T) \times(\xi, b) ; L^{2}(\Omega)\right)$ satisfying $\left.\phi\right|_{\theta=\xi}=0$ and $\left.\phi\right|_{t=T}=0$ and then extending them by zero for $a<\theta<\xi$ in the definition of weak solution to (3.1), we see that $z$ satisfies the weak form of (3.1) for $\theta \in(\xi, b)$. Consequently, $\left.z\right|_{\theta=\xi} \in L^{2}((0, T) \times \Omega)$ in the case of Theorem 3.2, while $\left.z\right|_{\theta=\xi} \in H^{m}\left(0, T ; H^{s}(\Omega)\right)$ in the case of Theorem 3.3.

\section{Existence of Weak Solutions}

The weak formulation of (1.1) will be posed in the state space $X=H_{f} \times H^{1}\left(\Omega_{s}\right) \times$ $L^{2}\left(\Omega_{s}\right) \times L^{2}\left((-r, 0) \times \Omega_{s}\right)$ and will be coupled in terms of the fluid and solid components. This is the same framework utilized in [28] for the case without delay. Thanks to the trace regularity for transport equations, it is advantageous to have test functions for the fluidstructure dynamics that are uncoupled to the delay variable. Without delay, an alternative direction was followed in [10] using the theory of monotone operators. This time the test functions for the fluid and solid components are decoupled due to hidden trace regularity for the hyperbolic part. We prefer the former approach, specially in establishing uniqueness and regularity in two dimensions. For the rest of the paper, we let $z^{\xi}:=\left.z\right|_{\theta=-\xi}$ whenever $\xi \in[0, r]$.

Definition 4.1. Given $T>0, G_{f} \in L^{2}\left(0, T ; V_{f}^{\prime}\right), G_{s} \in L^{2}\left(0, T ; L^{2}\left(\Omega_{s}\right)\right)$ and $\left(u_{0}, w_{0}, v_{0}, z_{0}\right) \in$ $X$, the quadruple $\left(u, w, w_{t}, z\right) \in L^{2}(0, T ; X)$ is called a weak solution of the fluid-structure model (1.1)-(1.2) if the following conditions are satisfied:

(a) $u \in L^{2}\left(0, T ; V_{f}\right)$

(b) $\left.u\right|_{\Gamma_{s}}=\left.w_{t}\right|_{\Gamma_{s}} \in L^{2}\left(0, T ; H^{\frac{1}{2}}\left(\Gamma_{s}\right)\right)$

(c) $z^{r_{d}} \in L^{2}\left(0, T ; L^{2}\left(\Omega_{s}\right)\right)$

(d) $w(0)=w_{0}$ 
(e) For every $(\varphi, \psi) \in H^{1}\left(0, T ; H_{0}\right) \cap L^{2}\left(0, T ; H_{1}\right)$ such that $\left.(\varphi, \psi)\right|_{t=T}=0$ we have

$$
\begin{aligned}
& \int_{0}^{T}\left\{-\left(u, \varphi_{t}\right)_{\Omega_{f}}+(\nabla u, \nabla \varphi)_{\Omega_{f}}+\langle(u \cdot \nabla) u, \varphi\rangle_{V_{f}^{\prime} \times V_{f}}-\frac{1}{2}\langle(u \cdot \nu) u, \varphi\rangle_{V_{f}^{\prime} \times V_{f}}\right\} \mathrm{d} t \\
& +\int_{0}^{T}\left\{-\left(w_{t}, \psi_{t}\right)_{\Omega_{s}}+(\sigma(w), \epsilon(\psi))_{\Omega_{s}}+\left(w+\mu w_{t}+\mu_{d} z^{r_{d}}, \psi\right)_{\Omega_{s}}\right\} \mathrm{d} t \\
& +\int_{0}^{T} \int_{-r_{c}}^{0} \mu_{c}(z, \psi)_{\Omega_{s}} \mathrm{~d} \theta \mathrm{d} t=\left(u_{0}, \varphi(0)\right)_{\Omega_{f}}+\left(v_{0}, \psi(0)\right)_{\Omega_{s}} \\
& +\int_{0}^{T}\left\{\left\langle G_{f}, \varphi\right\rangle_{V_{f}^{\prime} \times V_{f}}+\left(G_{s}, \psi\right)_{\Omega_{s}}\right\} \mathrm{d} t .
\end{aligned}
$$

(f) For every $\phi \in H^{1}\left((0, T) \times(-r, 0) ; L^{2}\left(\Omega_{s}\right)\right)$ with $\left.\phi\right|_{t=T}=0$ and $\phi^{r}=0$ we have

$$
-\int_{0}^{T} \int_{-r}^{0}\left(z, \phi_{t}-\phi_{\theta}\right)_{\Omega_{s}} \mathrm{~d} \theta \mathrm{d} t-\int_{0}^{T}\left(w_{t}, \phi^{0}\right)_{\Omega_{s}} \mathrm{~d} t=\int_{-r}^{0}\left(z_{0}, \phi(0)\right)_{\Omega_{s}} \mathrm{~d} \theta
$$

Let us give a few remarks with regards to the above definition. As a consequence of the definition, we have $w \in C\left(0, T ; L^{2}\left(\Omega_{s}\right)\right)$, so that (d) makes sense. Moreover, we have at the very least that $\left.w_{t}\right|_{\Gamma_{s}} \in H^{-1}\left(0, T ; H^{\frac{1}{2}}\left(\Gamma_{s}\right)\right)$. Nevertheless, condition (b) implies that we have more regularity. Condition (f) implies that $z$ is the weak solution of

$$
\begin{cases}z_{t}(t, \theta, x)-z_{\theta}(t, \theta, x)=0, & \text { in }(0, T) \times(-r, 0) \times \Omega, \\ z(t, 0, x)=w_{t}(t, x), & \text { in }(0, T) \times \Omega, \\ z(0, \theta, x)=z_{0}(\theta, x), & \text { in }(-r, 0) \times \Omega,\end{cases}
$$

and based on Remark 3.4, we obtain (c).

In the following, we shall prove well-posedness for every damping and delay factors $\left(\mu, \mu_{d}, \mu_{c}\right) \in \mathbb{R} \times \mathbb{R} \times L^{2}\left(-r_{c}, 0\right)$. In the linear case where semigroup methods are amenable, the corresponding result can be achieved from the bounded perturbation theorem for strongly continuous semigroups. In the nonlinear case, we shall do a similar process by replacing the test function $(\varphi, \psi)$ in condition (e) by $e^{-\gamma t}(\varphi, \psi)$ with a suitable $\gamma>0$. Notice that this map is an isomorphism of $H^{1}\left(0, T ; H_{0}\right) \cap L^{2}\left(0, T ; H_{1}\right)$ onto itself.

The effect of the above change of test functions with respect to the weak form is equivalent to the change of the unknowns $\left(u, w, w_{t}, p\right)$ to $e^{-\gamma t}\left(u, w, w_{t}, p\right)$ in the strong form. For this reason, let us define the new state variables $\left(u_{\gamma}, w_{\gamma}, v_{\gamma}, p_{\gamma}\right)=e^{-\gamma t}\left(u, w, w_{t}, p\right)$ as well as the rescaled source terms $G_{f \gamma}=e^{-\gamma t} G_{f}$ and $G_{s \gamma}=e^{-\gamma t} G_{s}$. The strong form of the Navier-Stokes equation becomes

$$
\begin{aligned}
u_{\gamma t}-\Delta u_{\gamma}+\gamma u_{\gamma}+e^{\gamma t}\left(u_{\gamma} \cdot \nabla\right) u_{\gamma}+\nabla p_{\gamma} & =G_{f \gamma}, \\
\operatorname{div} u_{\gamma} & =0,
\end{aligned}
$$

and using

$$
w_{\gamma t}=v_{\gamma}-\gamma w_{\gamma},
$$

the strong form for the elasticity equation turns into

$$
v_{\gamma t}-\operatorname{div} \sigma\left(w_{\gamma}\right)+w_{\gamma}+(\gamma+\mu) v_{\gamma}+\mu_{d} e^{-\gamma t} z^{r_{d}}+\int_{-r_{c}}^{0} e^{-\gamma t} \mu_{c} z \mathrm{~d} \theta=G_{s \gamma} .
$$

The boundary conditions remain the same for the rescaled variables, that is, $u_{\gamma}=v_{\gamma}$ on $\Gamma_{s}$ and $u_{\gamma}=0$ on $\Gamma_{f}$, except for the normal stresses on $\Gamma_{s}$ which becomes

$$
\frac{\partial u_{\gamma}}{\partial \nu}-p_{\gamma} \nu+\frac{1}{2} e^{\gamma t}\left(u_{\gamma} \cdot \nu\right) u_{\gamma}=\sigma\left(w_{\gamma}\right) \cdot \nu .
$$


Applying the variation of parameters formula to (4.2), we obtain $w_{\gamma}=e^{-\gamma t} w_{0}+J_{\gamma} v_{\gamma}$ where $J_{\gamma}$ is the integral operator

$$
\left(J_{\gamma} v\right)(t)=\int_{0}^{t} e^{-\gamma(t-s)} v(s) \mathrm{d} s .
$$

We are now going to write the rescaled variational equation in (e). First, let us define the bilinear forms $a_{f}: H^{1}\left(\Omega_{f}\right) \times H^{1}\left(\Omega_{f}\right) \rightarrow \mathbb{R}$ and $a_{s}: H^{1}\left(\Omega_{s}\right) \times H^{1}\left(\Omega_{s}\right) \rightarrow \mathbb{R}$ and the trilinear form $b: H^{1}\left(\Omega_{f}\right) \times H^{1}\left(\Omega_{f}\right) \times H^{1}\left(\Omega_{f}\right) \rightarrow \mathbb{R}$ respectively according to

$$
\begin{aligned}
& a_{f}(u, \varphi)=(\nabla u, \nabla \varphi)_{\Omega_{f}} \\
& a_{s}(w, \psi)=(\sigma(w), \epsilon(\psi))_{\Omega_{s}}+(w, \psi)_{\Omega_{s}} \\
& b(u, v, \varphi)=\langle(u \cdot \nabla) v, \varphi\rangle_{V_{f}^{\prime} \times V_{f}}-\frac{1}{2}\langle(u \cdot \nu) v, \varphi\rangle_{V_{f}^{\prime} \times V_{f}} .
\end{aligned}
$$

From the above discussions, we see that the variational equation in (e) can be rewritten as follows

$$
\begin{aligned}
& \int_{0}^{T}\left\{-\left(u_{\gamma}, \varphi_{t}\right)_{\Omega_{f}}+a_{f}\left(u_{\gamma}, \varphi\right)+\gamma\left(u_{\gamma}, \varphi\right)_{\Omega_{f}}+e^{\gamma t} b\left(u_{\gamma}, u_{\gamma}, \varphi\right)\right\} \mathrm{d} t \\
& +\int_{0}^{T}\left\{-\left(v_{\gamma}, \psi_{t}\right)_{\Omega_{s}}+a_{s}\left(J_{\gamma} v_{\gamma}, \psi\right)+\left((\gamma+\mu) v_{\gamma}+\mu_{d} e^{-\gamma t} z^{r_{d}}, \psi\right)_{\Omega_{s}}\right\} \mathrm{d} t \\
& +\int_{0}^{T} \int_{-r_{c}}^{0} e^{-\gamma t} \mu_{c}(z, \psi)_{\Omega_{s}} \mathrm{~d} \theta \mathrm{d} t=\left(u_{0}, \varphi(0)\right)_{\Omega_{f}}+\left(v_{0}, \psi(0)\right)_{\Omega_{s}} \\
& -\int_{0}^{T} e^{-\gamma t} a_{s}\left(w_{0}, \psi\right) \mathrm{d} t+\int_{0}^{T}\left\{\left\langle G_{f \gamma}, \varphi\right\rangle_{V_{f}^{\prime} \times V_{f}}+\left(G_{s \gamma}, \psi\right)_{\Omega_{s}}\right\} \mathrm{d} t,
\end{aligned}
$$

while the variational equation in (f) can be rewritten as

$$
-\int_{0}^{T} \int_{-r}^{0}\left(z, \phi_{t}-\phi_{\theta}\right)_{\Omega_{s}} \mathrm{~d} \theta \mathrm{d} t-\int_{0}^{T}\left(e^{\gamma t} v_{\gamma}, \phi^{0}\right)_{\Omega_{s}} \mathrm{~d} t=\int_{-r}^{0}\left(z_{0}, \phi(0)\right)_{\Omega_{s}} \mathrm{~d} \theta .
$$

Note that we only did a change of test functions in (e) and not in (f). If $\left(u_{\gamma}, v_{\gamma}, z\right) \in$ $L^{2}\left(0, T ; H_{f} \times L^{2}\left(\Omega_{s}\right) \times L^{2}\left((-r, 0) \times \Omega_{s}\right)\right)$ satisfies these new variational equations and $J_{\gamma} v_{\gamma} \in$ $L^{2}\left(0, T ; H^{1}\left(\Omega_{s}\right)\right)$ then it is not hard to see that $\left(u, w, w_{t}, z\right)$ with $u=e^{\gamma t} u_{\gamma}$ and $w=$ $e^{\gamma t} J_{\gamma} v_{\gamma}+w_{0}$ is a weak solution of (1.1)-(1.2).

Remark 4.2. An auxiliary problem may be considered in terms of the global velocity vector field $\xi_{\gamma}=u_{\gamma} \chi_{\Omega_{f}}+v_{\gamma} \chi_{\Omega_{s}}$ as in [16]. One proves the existence of $\xi_{\gamma}$ by FaedoGalerkin method. The solution of the original problem can be obtained by the restrictions $u_{\gamma}=\left.\xi_{\gamma}\right|_{\Omega_{f}}$ and $v_{\gamma}=\left.\xi_{\gamma}\right|_{\Omega_{s}}$. We prefer the formulation where the velocities are defined separately. Nevertheless, these two approaches are equivalent.

In the following lemma, we list some properties of the trilinear form $b$ that will be needed in the proof of existence of weak solutions. For their proofs, we refer to [10].

Lemma 4.3. Let $u \in V_{f}$ and $v, \varphi \in H^{1}\left(\Omega_{f}\right)$. Then we have the following:

(i) $b(u, v, \varphi)=-b(u, \varphi, v)$ and in particular $b(u, u, u)=0$

(ii) If $d=2$ then there exists a constant $C>0$ independent of $(u, v, \varphi)$ such that

$$
|b(u, v, \varphi)| \leq C\|u\|_{H^{\frac{1}{2}\left(\Omega_{f}\right)}}\left(\|v\|_{H^{1}\left(\Omega_{f}\right)}\|\varphi\|_{H^{\frac{1}{2}\left(\Omega_{f}\right)}}+\|v\|_{H^{\frac{3}{4}}\left(\Omega_{f}\right)}\|\varphi\|_{H^{\frac{3}{4}}\left(\Omega_{f}\right)}\right) .
$$


(iii) If $d=3$ then there exists a constant $C>0$ independent of $(u, v, \varphi)$ such that

$$
\begin{aligned}
& |b(u, v, \varphi)| \leq C\|u\|_{H^{\frac{1}{2}\left(\Omega_{f}\right)}}\|v\|_{H^{1}\left(\Omega_{f}\right)}\|\varphi\|_{H^{1}\left(\Omega_{f}\right)}\|v\|_{H^{1}\left(\Omega_{f}\right)}\|\varphi\|_{H^{\frac{3}{4}}\left(\Omega_{f}\right)} . \\
& |b(u, v, \varphi)| \leq C\|u\|_{H^{\frac{3}{4}}\left(\Omega_{f}\right)}
\end{aligned}
$$

The following consequence of this lemma will be utilized frequently in this paper.

Corollary 4.4. Let $d=2$. For every $\varepsilon>0$ there exists a constant $C_{\varepsilon}>0$ such that

$$
|b(u, v, u)| \leq \varepsilon\|u\|_{V_{f}}^{2}+C_{\varepsilon}\|u\|_{\Omega_{f}}^{2}\|v\|_{V_{f}}^{2}\left(1+\|v\|_{\Omega_{f}}^{2 / 3}\right)
$$

for every $u, v \in V_{f}$.

Proof. From Lemma 4.3(ii), interpolation for Sobolev spaces and Poincaré inequality

$$
|b(u, v, u)| \leq C\|u\|_{\Omega_{f}}\|u\|_{V_{f}}\|v\|_{V_{f}}+C\|u\|_{\Omega_{f}}^{3 / 4}\|u\|_{V_{f}}^{5 / 4}\|v\|_{\Omega_{f}}^{1 / 4}\|v\|_{V_{f}}^{3 / 4} .
$$

The desired estimate now follows by applying Young's inequality $a b \leq \frac{\varepsilon}{2} a^{p}+C_{p, \varepsilon} b^{p /(p-1)}$ with $a=\|u\|_{V_{f}}, b=C\|u\|_{\Omega_{f}}\|v\|_{V_{f}}, p=2$ and $a=\|u\|_{V_{f}}^{5 / 4}, b=C\|u\|_{\Omega_{f}}^{3 / 4}\|v\|_{\Omega_{f}}^{1 / 4}\|v\|_{V_{f}}^{3 / 4}$, $p=8 / 5$ to the first and second terms on the right hand side of (4.3), respectively.

Theorem 4.5. Let $T>0$ and set $V_{f, 0}=\left\{u \in V_{f}: u=0\right.$ on $\left.\Gamma_{s}\right\}$. Given $\left(u_{0}, w_{0}, v_{0}, z_{0}\right) \in$ $X, G_{f} \in L^{2}\left(0, T ; V_{f}^{\prime}\right), G_{s} \in L^{2}\left(0, T ; L^{2}\left(\Omega_{s}\right)\right)$ and $\left(\mu, \mu_{d}, \mu_{c}\right) \in \mathbb{R}^{2} \times L^{2}(-r, 0)$, the coupled system (1.1)-(1.2) has a weak solution. In addition, it holds that $\left(u, w, w_{t}\right) \in L^{\infty}\left(0, T ; H_{f} \times\right.$ $\left.H^{1}\left(\Omega_{s}\right) \times L^{2}\left(\Omega_{s}\right)\right), u_{t} \in L^{p}\left(0, T ; V_{f, 0}^{\prime}\right)$, where $p=2$ if $d=2$ and $p=\frac{4}{3}$ if $d=3$, wtt $\in$ $L^{2}\left(0, T ; H^{-1}\left(\Omega_{s}\right)\right)$, and $z \in C\left(0, T ; L^{2}\left((-r, 0) \times \Omega_{s}\right)\right) \cap L^{2}\left(\Omega_{s} ; V_{L}^{2}\left(Q_{T}\right)\right)$.

Proof. We apply a standard Faedo-Galerkin method using the orthonormal basis of $H_{0}$ obtained from the Stokes-Neumann operator $A$. For each positive integer $m$, define

$$
\begin{array}{ll}
u_{m}(t, x)=\sum_{j=1}^{m} \alpha_{j m}(t) \varphi_{j}(x), & (t, x) \in(0, T) \times \Omega_{f}, \\
v_{m}(t, x)=\sum_{j=1}^{m} \alpha_{j m}(t) \psi_{j}(x), & (t, x) \in(0, T) \times \Omega_{s} .
\end{array}
$$

The continuity on the interface $\left.u_{m}\right|_{\Gamma_{s}}=\left.v_{m}\right|_{\Gamma_{s}}$ holds since $\left.\varphi_{j}\right|_{\Gamma_{s}}=\left.\psi_{j}\right|_{\Gamma_{s}}$ for each $j$. Consider the following approximate system of integro-differential delay equations

$$
\begin{aligned}
& \dot{\alpha}_{i m}(t)+\sum_{j=1}^{m} \alpha_{j m}(t)\left\{a_{f}\left(\varphi_{j}, \varphi_{i}\right)+\gamma\left(\varphi_{j}, \varphi_{i}\right)_{\Omega_{f}}\right\}+\sum_{j, k=1}^{m} \alpha_{j m}(t) \alpha_{k m}(t) e^{\gamma t} b\left(\varphi_{j}, \varphi_{k}, \varphi_{i}\right) \\
& +\sum_{j=1}^{m}\left(J_{\gamma} \alpha_{j m}\right)(t) a_{s}\left(\psi_{j}, \psi_{i}\right)+\sum_{j=1}^{m}\left\{(\gamma+\mu) \alpha_{j m}(t)+\mu_{d} e^{-\gamma t} h_{j m}\left(t,-r_{d}\right)\right\}\left(\psi_{j}, \psi_{i}\right)_{\Omega_{s}} \\
& +\sum_{j=1}^{m} \int_{-r_{c}}^{0} e^{-\gamma t} \mu_{c}(\theta) h_{j m}(t, \theta)\left(\psi_{j}, \psi_{i}\right)_{\Omega_{s}} \mathrm{~d} \theta=\left\langle G_{f \gamma}(t), \varphi_{i}\right\rangle_{V_{f}^{\prime} \times V_{f}}+\left(G_{s \gamma}(t), \psi_{i}\right)_{\Omega_{s}} \\
& -e^{-\gamma t} a_{s}\left(w_{0}, \psi_{i}\right), \quad \alpha_{i m}(0)=\alpha_{i m 0},
\end{aligned}
$$

where $h_{i m}$ is the solution of the following transport equation

$$
\partial_{t} h_{i m}(t, \theta)-\partial_{\theta} h_{i m}(t, \theta)=0, \quad h_{i m}(t, 0)=e^{\gamma t} \alpha_{j m}(t), \quad h_{i m}(0, \theta)=h_{i m 0}(\theta),
$$


for $(t, \theta, x) \in(0, T) \times(-r, 0) \times \Omega_{s}$ and for $i=1, \ldots, m$. The corresponding approximate initial data and history are given by

$$
\begin{aligned}
& \alpha_{i m}(0)=\left(u_{0}, \varphi_{i}\right)_{\Omega_{f}}+\left(v_{0}, \psi_{i}\right)_{\Omega_{s}}, \\
& h_{i m 0}(\theta)=\left(z_{0}(\theta), \psi_{i}\right)_{\Omega_{s}}, \quad \theta \in(-r, 0) \text {. }
\end{aligned}
$$

Let $\alpha_{m}=\left(\alpha_{1 m}, \ldots, \alpha_{m m}\right)^{T}, h_{m}=\left(h_{1 m}, \ldots, h_{m m}\right)^{T}$ and $f_{m}=J_{\gamma} \alpha_{m}$. Likewise, define the matrices $A_{m}=\left[a_{f}\left(\varphi_{j}, \varphi_{i}\right)+\gamma\left(\varphi_{j}, \varphi_{i}\right)_{\Omega_{f}}+(\gamma+\mu)\left(\psi_{j}, \psi_{i}\right)_{\Omega_{s}}\right]_{i, j=1}^{m}, C_{m}=\left[a_{s}\left(\psi_{j}, \psi_{i}\right)\right]_{i, j=1}^{m}$, $D_{m}=\left[\left(\psi_{j}, \psi_{i}\right)_{\Omega_{s}}\right]_{i, j=1}^{m}$ and $g_{m}=\left[\left\langle G_{f \gamma}, \varphi_{i}\right\rangle_{V_{f}^{\prime} \times V_{f}}+\left(G_{s \gamma}, \psi_{i}\right)_{\Omega_{s}}-e^{-\gamma t} a_{s}\left(w_{0}, \psi_{i}\right)\right]_{i=1}^{m}$. The equations (4.4) can be written as the system

$$
\left\{\begin{array}{l}
\dot{\alpha}_{m}(t)+A_{m} \alpha_{m}(t)+C_{m} f_{m}(t)+e^{\gamma t} N_{m}\left(\alpha_{m}(t)\right)+\mu_{d} e^{-\gamma t} D_{m} h_{m}\left(t,-r_{d}\right) \\
\quad+\int_{-r_{c}}^{0} e^{-\gamma t} \mu_{c}(\vartheta) D_{m} h_{m}(t, \vartheta) \mathrm{d} \theta=g_{m}(t) \\
\dot{f}_{m}(t)-f_{m}(t)-\gamma \alpha_{m}(t)=0
\end{array}\right.
$$

for the unknowns $\left(\alpha_{m}, f_{m}, h_{m}\right)$, where $N_{m}$ is the nonlinear function associated with the trilinear form $b$. From the theory of delay differential equations, system (4.5)-(4.6) admits a unique local solution with $h_{m} \in C\left(0, T_{m} ; L^{2}(-r, 0)\right) \cap V_{L}\left(Q_{T_{m}}\right), \alpha_{m} \in H^{1}\left(0, T_{m}\right)$ and $f_{m} \in H^{2}\left(0, T_{m}\right)$ for some $T_{m} \in(0, T]$. We either have $T_{m}=T$ or $\left|\alpha_{m}(t)\right|+\left|f_{m}(t)\right|+$ $\left\|h_{m}(t)\right\|_{L^{2}(-r, 0)} \rightarrow \infty$ as $t \rightarrow T_{m}$. The a priori estimates below implies that the second case is not possible, hence $T_{m}=T$.

Define $z_{m}=\sum_{j=1}^{m} h_{j m} \psi_{j}$ and $z_{m 0}=\sum_{j=1}^{m} h_{j m 0} \psi_{j}$. Note that $\left.z_{m}\right|_{\theta=0}=e^{\gamma t} v_{m}$ by (4.5). It follows from (4.5) that for each $\xi \in[0, r], z_{m}$ satisfies

$$
\begin{aligned}
& \frac{1}{2} \frac{d}{d t} \int_{-\xi}^{0} e^{-2 \gamma t}\left\|z_{m}(t, \theta)\right\|_{\Omega_{s}}^{2} \mathrm{~d} \theta \\
& +\gamma \int_{-\xi}^{0} e^{-2 \gamma t}\left\|z_{m}(t, \theta)\right\|_{\Omega_{s}}^{2} \mathrm{~d} \theta+\frac{1}{2} e^{-2 \gamma t}\left\|z_{m}^{\xi}(t)\right\|_{\Omega_{s}}^{2}=\frac{1}{2}\left\|v_{m}(t)\right\|_{\Omega_{s}}^{2}
\end{aligned}
$$

Let $w_{m}=e^{-\gamma t} w_{0}+J_{\gamma} v_{m}$ so that $w_{m t}=v_{m}-\gamma w_{m}$. Multiplying (4.4) by $\alpha_{i m}$, adding the resulting terms for $i=1, \ldots, m$ and using

$$
\frac{1}{2} \frac{d}{d t} a_{s}\left(w_{m}(t), w_{m}(t)\right)=a_{s}\left(w_{m}(t), v_{m}(t)\right)-\gamma a_{s}\left(w_{m}(t), w_{m}(t)\right)
$$

we have

$$
\begin{aligned}
& \frac{1}{2} \frac{d}{d t}\left(\left\|u_{m}(t)\right\|_{\Omega_{f}}^{2}+\left\|v_{m}(t)\right\|_{\Omega_{s}}^{2}+a_{s}\left(w_{m}(t), w_{m}(t)\right)\right)+a_{f}\left(u_{m}(t), u_{m}(t)\right) \\
& +\gamma\left\|u_{m}(t)\right\|_{\Omega_{f}}^{2}+\gamma a_{s}\left(w_{m}(t), w_{m}(t)\right)+(\gamma+\mu)\left\|v_{m}(t)\right\|_{\Omega_{s}}^{2} \\
& +\left(e^{-\gamma t} z_{m}^{r_{d}}(t), \mu_{d} v_{m}(t)\right)_{\Omega_{s}}+\int_{-r_{c}}^{0} \mu_{c}(\theta)\left(e^{-\gamma t} z_{m}(t, \theta), v_{m}(t)\right)_{\Omega_{s}} \mathrm{~d} \theta \\
& =\left\langle G_{f \gamma}(t), u_{m}(t)\right\rangle_{V_{f}^{\prime} \times V_{f}}+\left(G_{s \gamma}(t), v_{m}(t)\right)_{\Omega_{s}} .
\end{aligned}
$$

Here, we used $b\left(u_{m}, u_{m}, u_{m}\right)=0$ according to Lemma 4.3(i). 
Taking the sum of (4.7) with $\xi=r_{d}, \xi=r_{c}$ and (4.8), integrating with respect to time and applying the Cauchy-Schwarz inequality, we obtain

$$
\begin{aligned}
& e_{m}(t)+(1-\varepsilon) \int_{0}^{t} a_{f}\left(u_{m}(\sigma), u_{m}(\sigma)\right) \mathrm{d} \sigma \\
& +\gamma \int_{0}^{t} a_{s}\left(w_{m}(\sigma), w_{m}(\sigma)\right) \mathrm{d} \sigma+C_{\varepsilon, \gamma} \int_{0}^{t}\left\|v_{m}(\sigma)\right\|_{\Omega_{s}}^{2} \mathrm{~d} \sigma \\
& +\left(\frac{1}{2}-\varepsilon\right) \int_{0}^{t} e^{-2 \gamma \sigma}\left\|z_{m}^{r_{d}}(\sigma)\right\|_{\Omega_{s}}^{2} \mathrm{~d} \sigma+\left(\gamma-\frac{1}{2}\right) \int_{0}^{t} \int_{-r_{c}}^{0} e^{-2 \gamma \sigma}\left\|z_{m}(\sigma, \theta)\right\|_{\Omega_{s}}^{2} \mathrm{~d} \theta \mathrm{d} \sigma \\
& \leq e_{m}(0)+\frac{1}{2} \int_{0}^{t}\left(C_{\varepsilon}\left\|G_{f \gamma}(\sigma)\right\|_{V_{f}^{\prime}}^{2}+\left\|G_{s \gamma}(\sigma)\right\|_{\Omega_{s}}^{2}\right) \mathrm{d} \sigma
\end{aligned}
$$

where $\varepsilon, C, C_{\varepsilon}>0, C_{\varepsilon, \gamma}=\gamma+\mu-1-\mu_{d}^{2} C_{\varepsilon}-\frac{1}{2}\left\|\mu_{c}\right\|_{L^{2}\left(-r_{c}, 0\right)}^{2}$ and

$$
\begin{aligned}
e_{m}(t)= & \frac{1}{2}\left(\left\|u_{m}(t)\right\|_{\Omega_{f}}^{2}+\left\|v_{m}(t)\right\|_{\Omega_{s}}^{2}+a_{s}\left(w_{m}(t), w_{m}(t)\right)\right) \\
& +\frac{1}{2} \int_{-r_{d}}^{0} e^{-2 \gamma t}\left\|z_{m}(t, \theta)\right\|_{\Omega_{s}}^{2} \mathrm{~d} \theta+\frac{1}{2} \int_{-r_{c}}^{0} e^{-2 \gamma t}\left\|z_{m}(t, \theta)\right\|_{\Omega_{s}}^{2} \mathrm{~d} \theta .
\end{aligned}
$$

By definition, we have $\left(u_{m}(0), v_{m}(0)\right)=\Pi_{m}\left(u_{0}, v_{0}\right), J_{\gamma} v_{m}(0)=0$ and $z_{m}(0, \theta)=$ $\left.\Pi_{m}\left(0, z_{0}(\theta)\right)\right|_{\Omega_{s}}$. The boundedness of the projections $\Pi_{m}$ imply that $e_{m}(0)$ is uniformly bounded with respect to $m$. By choosing $\varepsilon$ small enough then $\gamma$ large enough so that $C_{\varepsilon, \gamma}>0$, we obtain the following

$$
\begin{cases}\left(u_{m}\right)_{m} & \text { bounded in } L^{\infty}\left(0, T ; H_{f}\right) \cap L^{2}\left(0, T ; V_{f}\right), \\ \left(v_{m}\right)_{m} & \text { bounded in } L^{\infty}\left(0, T ; L^{2}\left(\Omega_{s}\right)\right), \\ \left(w_{m}\right)_{m} & \text { bounded in } L^{\infty}\left(0, T ; H^{1}\left(\Omega_{s}\right)\right), \\ \left(z_{m}\right)_{m} & \text { bounded in } L^{\infty}\left(0, T ; L^{2}\left((-r, 0) \times \Omega_{s}\right)\right), \\ \left(z_{m}^{r_{d}}\right)_{m} & \text { bounded in } L^{2}\left(0, T ; L^{2}\left(\Omega_{s}\right)\right) .\end{cases}
$$

With these bounds, we obtain the following convergences after an extraction of an appropriate subsequence. First, we have $u_{m} \rightarrow u_{\gamma}$ in $L^{2}\left(0, T ; V_{f}\right), v_{m} \rightarrow v_{\gamma}$ in $L^{2}\left(0, T ; L^{2}\left(\Omega_{s}\right)\right)$, $w_{m} \rightarrow w_{\gamma}$ in $L^{2}\left(0, T ; H^{1}\left(\Omega_{s}\right)\right), z_{m} \rightarrow z$ in $L^{2}\left(0, T ; L^{2}\left((-r, 0) \times \Omega_{s}\right)\right)$ and $z_{m}^{r_{d}} \rightarrow \zeta$ in $L^{2}\left(0, T ; L^{2}\left(\Omega_{s}\right)\right)$. Since $u_{m t}$ is bounded in $L^{p}\left(0, T ; V_{f, 0}^{\prime}\right)$ and the embedding $V_{f, 0} \subset H_{f}$ is compact, we have $u_{m} \rightarrow u_{\gamma}$ in $L^{2}\left(0, T ; H_{f}\right)$ by Aubin-Lions-Simon Lemma [40]. The integral operator $J_{\gamma}$ is bounded from $L^{2}\left(0, T ; L^{2}\left(\Omega_{s}\right)\right)$ into itself, thus we have $J_{\gamma} v_{m} \rightarrow J_{\gamma} v_{\gamma}$ in $L^{2}\left(0, T ; L^{2}\left(\Omega_{s}\right)\right)$, and as a result $w_{\gamma}=J_{\gamma} v_{\gamma}+e^{-\gamma t} w_{0}$.

Since $\partial_{t} z-\partial_{\theta} z=0=\partial_{t} z_{m}-\partial_{\theta} z_{m}$ in the sense of distributions, we have $z_{m} \rightarrow z$ in $L^{2}\left(\Omega_{s} ; W_{L}^{2}\left(Q_{T}\right)\right)$. The continuity of the generalized trace implies that we have $z_{m}^{r_{d}} \rightarrow z^{r_{d}}$ in $L^{2}\left(\Omega_{s} ; V\left(\Sigma^{-r_{d}}\right)^{\prime}\right)$. We refer the reader to the Appendix for the definitions of the function spaces $W_{L}^{2}\left(Q_{T}\right)$ and $V\left(\Sigma^{-r_{d}}\right)^{\prime}$. By the uniqueness of weak limits, it follows that $\zeta=z^{r_{d}}$. To verify (b), first let us notice that $\left.\left.u_{m}\right|_{\Gamma_{s}} \rightarrow u_{\gamma}\right|_{\Gamma_{s}}$ in $L^{2}\left(0, T ; H^{\frac{1}{2}}\left(\Gamma_{s}\right)\right)$. From $\left.\left.J_{\gamma} v_{m}\right|_{\Gamma_{s}} \rightarrow w_{\gamma}\right|_{\Gamma_{s}}$ in $L^{2}\left(0, T ; H^{\frac{1}{2}}\left(\Gamma_{s}\right)\right)$, we obtain that $\left.v_{m}\right|_{\Gamma_{s}}=\left.\left.\left(\left(J_{\gamma} v_{m}\right)_{t}+\gamma J_{\gamma} v_{m}\right)\right|_{\Gamma_{s}} \rightarrow\left(w_{\gamma t}+\gamma w_{\gamma}\right)\right|_{\Gamma_{s}}=\left.v_{\gamma}\right|_{\Gamma_{s}}$ in $H^{-1}\left(0, T ; H^{\frac{1}{2}}\left(\Gamma_{s}\right)\right)$. Since $\left.u_{m}\right|_{\Gamma_{s}}=\left.v_{m}\right|_{\Gamma_{s}}$ for all $m$, it follows that $\left.u_{\gamma}\right|_{\Gamma_{s}}=\left.v_{\gamma}\right|_{\Gamma_{s}}$.

Given $\eta_{j} \in H^{1}(0, T)$ and $\rho_{j} \in H^{1}\left(Q_{T}\right)$ such that $\eta_{j}(T)=0,\left.\rho_{j}\right|_{t=T}=0$ and $\rho_{j}^{r}=0$, introduce the following test functions

$$
\varphi^{k}=\sum_{i=1}^{k} \eta_{i} \varphi_{i}, \quad \psi^{k}=\sum_{i=1}^{k} \eta_{i} \psi_{i}, \quad \phi^{k}=\sum_{i=1}^{k} \rho_{i} \psi_{i}
$$


for $1 \leq k \leq m$. Multiplying (4.4) by $\eta_{i}$, taking the sum for $1 \leq i \leq k$, and then integrating by parts over the time interval $[0, T]$, we have the rescaled variational equation in (e) with $\left(u_{\gamma}, v_{\gamma}, z\right),(\varphi, \psi)$ and $\left(u_{0}, v_{0}\right)$ replaced by $\left(u_{m}, v_{m}, z_{m}\right),\left(\varphi^{k}, \psi^{k}\right)$ and $\left(u_{m 0}, v_{m 0}\right)$, respectively. That is, for every $1 \leq k \leq m$ we have

$$
\begin{aligned}
& \int_{0}^{T}\left\{-\left(u_{m}, \varphi_{t}^{k}\right)_{\Omega_{f}}+a_{f}\left(u_{m}, \varphi^{k}\right)+\gamma\left(u_{m}, \varphi^{k}\right)_{\Omega_{f}}+e^{\gamma t} b\left(u_{m}, u_{m}, \varphi^{k}\right)\right\} \mathrm{d} t \\
& +\int_{0}^{T}\left\{-\left(v_{m}, \psi_{t}^{k}\right)_{\Omega_{s}}+a_{s}\left(J_{\gamma} v_{m}, \psi^{k}\right)+\left((\gamma+\mu) v_{m}+\mu_{d} e^{-\gamma t} z_{m}^{r_{d}}, \psi^{k}\right)_{\Omega_{s}}\right\} \mathrm{d} t \\
& +\int_{0}^{T} \int_{-r_{c}}^{0} e^{-\gamma t} \mu_{c}\left(z_{m}, \psi^{k}\right)_{\Omega_{s}} \mathrm{~d} \theta \mathrm{d} t=\left(u_{0 m}, \varphi^{k}(0)\right)_{\Omega_{f}}+\left(v_{0 m}, \psi^{k}(0)\right)_{\Omega_{s}} \\
& -\int_{0}^{T} e^{-\gamma t} a_{s}\left(w_{0}, \psi^{k}\right) \mathrm{d} t+\int_{0}^{T}\left\{\left\langle G_{f \gamma}, \varphi^{k}\right\rangle_{V_{f}^{\prime} \times V_{f}}+\left(G_{s \gamma}, \psi^{k}\right)_{\Omega_{s}}\right\} \mathrm{d} t
\end{aligned}
$$

where $\left(u_{0 m}, v_{0 m}\right)=\Pi_{m}\left(u_{0}, v_{0}\right)$. On the other hand, multiplying the first equation in (4.5) by $\psi_{i}$, taking the sum for $1 \leq i \leq m$, testing the resulting sum by $\phi^{k}$, and integrating by parts on $[0, T] \times(-r, 0)$, we obtain

$$
-\int_{0}^{T} \int_{-r}^{0}\left(z_{m}, \phi_{t}^{k}-\phi_{\theta}^{k}\right)_{\Omega_{s}} \mathrm{~d} \theta \mathrm{d} t-\int_{0}^{T}\left(e^{\gamma t} v_{m},\left.\phi^{k}\right|_{\theta=0}\right)_{\Omega_{s}} \mathrm{~d} t=\int_{-r}^{0}\left(z_{0 m}, \phi^{k}(0)\right)_{\Omega_{s}} \mathrm{~d} \theta
$$

where $z_{0 m}(\theta)=\left.\Pi_{m}\left(0, z_{0}(\theta)\right)\right|_{\Omega_{s}}$, which corresponds to the equation in (f), where $z$ and $z_{0}$ replaced by $z_{m}$ and $z_{0 m}$, respectively.

Writing $b\left(u_{m}, u_{m}, \varphi^{k}\right)-b\left(u_{\gamma}, u_{\gamma}, \varphi^{k}\right)=b\left(u_{m}-u_{\gamma}, u_{m}, \varphi^{k}\right)-b\left(u_{\gamma}, u_{m}-u_{\gamma}, \varphi^{k}\right)$ and using Lemma 4.3(iii) and interpolation estimates, we have

$$
\begin{aligned}
& \left|\int_{0}^{T} e^{\gamma t}\left(b\left(u_{m}, u_{m}, \varphi^{k}\right)-b\left(u_{\gamma}, u_{\gamma}, \varphi^{k}\right)\right) \mathrm{d} t\right| \\
& \leq e^{\gamma T} \int_{0}^{T}\left(\left|b\left(u_{m}-u_{\gamma}, u_{m}, \varphi^{k}\right)\right|+\left|b\left(u_{\gamma}, u_{m}-u_{\gamma}, \varphi^{k}\right)\right|\right) \mathrm{d} t \\
& \leq C e^{\gamma T} \int_{0}^{T}\left\|u_{m}-u_{\gamma}\right\|_{H^{\frac{1}{2}}\left(\Omega_{f}\right)}\left\|\varphi^{k}\right\|_{V_{f}}\left(\left\|u_{m}\right\|_{V_{f}}+\left\|u_{\gamma}\right\|_{V_{f}}\right) \mathrm{d} t \\
& \leq C e^{\gamma T}\left\|\varphi^{k}\right\|_{L^{\infty}\left(0, T ; V_{f}\right)} \int_{0}^{T}\left\|u_{m}-u_{\gamma}\right\|_{\Omega_{f}}^{\frac{1}{2}}\left\|u_{m}-u_{\gamma}\right\|_{V_{f}}^{\frac{1}{2}}\left(\left\|u_{m}\right\|_{V_{f}}+\left\|u_{\gamma}\right\|_{V_{f}}\right) \mathrm{d} t \\
& \leq C\left\|\varphi^{k}\right\|_{L^{\infty}\left(0, T ; V_{f}\right)}\left\|u_{m}-u_{\gamma}\right\|_{L^{2}\left(0, T ; H_{f}\right)}^{\frac{1}{2}}\left\|u_{m}-u_{\gamma}\right\|_{L^{2}\left(0, T ; V_{f}\right)}^{\frac{1}{2}}
\end{aligned}
$$

where $C=C\left(T,\left\|u_{m}\right\|_{L^{2}\left(0, T ; V_{f}\right)},\left\|u_{\gamma}\right\|_{L^{2}\left(0, T ; V_{f}\right)}\right)>0$. For fix $k$, this tends to zero as $m \rightarrow \infty$ according to the uniform boundedness of $u_{m}$ in $L^{\infty}\left(0, T ; H_{f}\right)$ and the fact that $u_{m} \rightarrow u_{\gamma}$ in $L^{2}\left(0, T ; H_{f}\right)$.

Observe that the set of all linear combinations of the form $\left(\eta \varphi_{j}, \eta \psi_{j}\right)$ for every $\eta \in$ $H^{1}(0, T)$ such that $\eta(T)=0$ is dense in $\left\{(\varphi, \psi) \in H^{1}\left(0, T ; H_{0}\right) \cap L^{2}\left(0, T ; H_{1}\right):\left.(\varphi, \psi)\right|_{t=T}=\right.$ $0\}$. Similarly, the set of all linear combinations of the form $\rho \psi_{j}$ for every $\rho \in H^{1}((0, T) \times$ $(-r, 0))$ with $\left.\rho\right|_{t=T}=0$ and $\rho^{r}=0$ is dense in $\left\{\phi \in H^{1}\left((0, T) \times(-r, 0) ; L^{2}\left(\Omega_{s}\right)\right):\left.\phi\right|_{t=T}=\right.$ $\left.0, \phi^{r}=0\right\}$. Passing to the limit $m \rightarrow \infty$ in (4.11) and (4.12), and then using these density properties, we obtain the rescaled versions of (e) and (f) in Definition (4.1). Furthermore, we since $u_{\gamma} \in L^{2}\left(0, T ; V_{f}\right),\left.u_{\gamma}\right|_{\Gamma_{s}}=\left.v_{\gamma}\right|_{\Gamma_{s}}$ and $w_{\gamma}(0)=w_{0}$, we also (a), (b) and (d).

As has been noted, $z$ is the weak solution of a parameter depending transport equation with boundary data $w_{t} \in L^{2}\left(0, T ; L^{2}\left(\Omega_{s}\right)\right)$ and initial data $z_{0} \in L^{2}\left((-r, 0) \times \Omega_{s}\right)$. 
Consequently, $z \in C\left(0, T ; L^{2}\left((-\tau, 0) \times \Omega_{s}\right)\right) \cap L^{2}\left(\Omega_{s} ; V_{L}^{2}\left(Q_{T}\right)\right)$ according to Theorem 3.2 and $z^{r_{d}} \in L^{2}\left(0, T ; L^{2}\left(\Omega_{s}\right)\right)$ by Remark 3.4, thus (c) is verified. The regularity of the time derivatives $u_{t} \in L^{p}\left(0, T ; V_{f, 0}^{\prime}\right)$, with $p$ as described in the statement of the theorem, and $w_{t t} \in L^{2}\left(0, T ; H^{-1}\left(\Omega_{s}\right)\right)$ follows immediately from the variational equation (e) by choosing test functions $\varphi \in L^{p /(p-1)}\left(0, T ; V_{f, 0}\right)$ and $\psi \in L^{2}\left(0, T ; H_{0}^{1}\left(\Omega_{s}\right)\right)$, respectively. Finally, by the sequential compactness of $L^{\infty}\left(0, T ; H_{f} \times H^{1}\left(\Omega_{s}\right) \times L^{2}\left(\Omega_{s}\right)\right)$ in the weak-star topology, we also have $\left(u, w, w_{t}\right) \in L^{\infty}\left(0, T ; H_{f} \times H^{1}\left(\Omega_{s}\right) \times L^{2}\left(\Omega_{s}\right)\right)$.

Given a Banach space $X$, denote by $C_{S}(0, T ; X)$ the set all functions $u \in L^{\infty}(0, T ; X)$ such that for each $f \in X^{\prime}$ the map $t \mapsto\langle f, u(t)\rangle_{X^{\prime} \times X}$ is continuous on $[0, T]$.

Theorem 4.6. The component $w$ of the weak solution of (1.1) constructed by the FaedoGalerkin approximations satisfies $w_{t} \in C_{S}\left(0, T ; L^{2}\left(\Omega_{s}\right)\right)$ and $w \in C_{S}\left(0, T ; H^{1}\left(\Omega_{s}\right)\right)$.

Proof. From the proof of existence, we have in fact the weak-star convergence of $w_{m}$ to $w$ in $L^{\infty}\left(0, T ; H^{1}\left(\Omega_{s}\right)\right)$ and $w_{m t}$ to $w_{t}$ in $L^{\infty}\left(0, T ; L^{2}\left(\Omega_{s}\right)\right)$. Since $w \in C\left(0, T ; L^{2}\left(\Omega_{s}\right)\right)$, it follows from $\left[\mathbf{2 9}\right.$, Lemma 8.1] that $w \in C_{S}\left(0, T ; H^{1}\left(\Omega_{s}\right)\right)$. Likewise, we have $w_{t t} \in$ $L^{2}\left(0, T ; H^{-1}\left(\Omega_{s}\right)\right)$, so that $w_{t} \in C\left(0, T ; H^{-1}\left(\Omega_{s}\right)\right)$, and thus $w_{t} \in C_{S}\left(0, T ; L^{2}\left(\Omega_{s}\right)\right)$.

\section{Uniqueness and Continuity of Weak Solutions in Two Dimensions}

In this section we shall establish the uniqueness of weak solutions in the two-dimensional case, and in addition, we prove that the weak solutions are continuous in time with values in the state space $X$.

Theorem 5.1. If $d=2$, then the weak solution of the fluid-structure system (1.1) is unique.

Proof. Suppose that $\left(u_{1}, v_{1}, z_{1}\right)$ and $\left(u_{2}, v_{2}, z_{2}\right)$ are two weak solutions of the rescaled weak forms of (e) and (f), and denote by $(u, v, z)$ the difference of these weak solutions. Then we have the variational equation

$$
\begin{aligned}
& \int_{0}^{T}\left\{-\left(u, \varphi_{t}\right)_{\Omega_{f}}+a_{f}(u, \varphi)+\gamma(u, \varphi)_{\Omega_{f}}+e^{\gamma t} B\left(u, u_{1}, \varphi\right)\right\} \mathrm{d} t \\
& +\int_{0}^{T}\left\{-\left(v, \psi_{t}\right)_{\Omega_{s}}+a_{s}\left(J_{\gamma} v, \psi\right)+\left((\gamma+\mu) v+\mu_{d} e^{-\gamma t} z^{r_{d}}, \psi\right)_{\Omega_{s}}\right\} \mathrm{d} t \\
& +\int_{0}^{T} \int_{-r_{c}}^{0} e^{-\gamma t} \mu_{c}(\theta)\left(z_{m}(\theta), \psi\right)_{\Omega_{s}} \mathrm{~d} \theta \mathrm{d} t=0
\end{aligned}
$$

for every $(\varphi, \psi) \in H^{1}\left(0, T ; H_{0}\right) \cap L^{2}\left(0, T ; H_{1}\right)$ such that $(\varphi(T), \psi(T))=(0,0)$, where the trilinear form $B$ is defined by $B\left(u, u_{1}, \varphi\right)=b\left(u_{1}, u, \varphi\right)+b\left(u, u_{1}, \varphi\right)-b(u, u, \varphi)$. The weak form for the transport equation for $z$ is the same due to linearity.

Fix $t \in(0, T)$ and let $\vartheta_{m}$ be the absolutely continuous piecewise linear function such that $\vartheta_{m}(s)=1$ for $\frac{2}{m} \leq s \leq t-\frac{2}{m}$ and $\vartheta_{m}(s)=0$ for $s>t-\frac{1}{m}$ or $s<\frac{1}{m}$. Let $\varrho_{n}$ be an even mollifier whose support lies in $\left[-\frac{1}{n}, \frac{1}{n}\right]$, that is, $\varrho_{n} \in C^{\infty}(\mathbb{R}), \varrho_{n}(s)=\varrho_{n}(-s)$ and $\int_{\mathbb{R}} \varrho_{n}(s) \mathrm{d} s=1$. For $n>2 m$ we consider the test functions

$$
\left(\varphi_{n m}, \psi_{n m}\right)=\vartheta_{m}\left(\left(\vartheta_{m} u\right) * \varrho_{n} * \varrho_{n},\left(\vartheta_{m} v\right) * \varrho_{n} * \varrho_{n}\right) \in H_{0}^{1}\left(0, T ; H_{1}\right)
$$


where $u$ and $v$ are extended by zero outside $[0, T]$ and $*$ denotes convolution with respect to time, see [28, Chapter 1]. Fixing $m$ and letting $n \rightarrow \infty$, we have

$$
\begin{aligned}
& \int_{0}^{t}\left(u, \varphi_{n m t}\right)_{\Omega_{f}} \mathrm{~d} s=\int_{0}^{t} \dot{\vartheta}_{m}\left(u * \varrho_{n},\left(\vartheta_{m} u\right) * \varrho_{n}\right)_{\Omega_{f}} \mathrm{~d} s \\
& \left.+\int_{0}^{t}\left(\left(\vartheta_{m} u\right) * \varrho_{n}\right)_{t},\left(\vartheta_{m} u\right) * \varrho_{n}\right)_{\Omega_{f}} \mathrm{~d} t \rightarrow \int_{0}^{t} \dot{\vartheta}_{m} \vartheta_{m}\|u\|_{\Omega_{f}}^{2} \mathrm{~d} s .
\end{aligned}
$$

In a similar way, we have the following

$$
\begin{aligned}
& \int_{0}^{t}\left(v, \psi_{n m t}\right) \mathrm{d} s \rightarrow \int_{0}^{t} \dot{\vartheta}_{m} \vartheta_{m}\|v\|_{\Omega_{s}}^{2} \mathrm{~d} t \\
& \int_{0}^{t}\left\{a_{f}\left(u, \varphi_{n m}\right)+\gamma\left(u, \varphi_{n m}\right)_{\Omega_{f}}+\left((\gamma+\mu) v+\mu_{d} e^{-\gamma s} z^{r_{d}}, \psi_{n m}\right)_{\Omega_{s}}\right\} \mathrm{d} s \\
& \quad \rightarrow \int_{0}^{t} \vartheta_{m}^{2}\left\{a_{f}(u, u)+\gamma(u, u)_{\Omega_{f}}+\left((\gamma+\mu) v+\mu_{d} e^{-\gamma s} z^{r_{d}}, v\right)_{\Omega_{s}}\right\} \mathrm{d} \theta \mathrm{d} s \\
& \int_{0}^{t} \int_{-r_{c}}^{0} e^{-\gamma s} \mu_{c}(\theta)\left(z_{m}(\theta), \psi_{n m}\right)_{\Omega_{s}} \mathrm{~d} \theta \mathrm{d} s \rightarrow \int_{0}^{t} \int_{-r_{c}}^{0} \vartheta_{m}^{2} e^{-\gamma s} \mu_{c}(\theta)(z(\theta), v)_{\Omega_{s}} \mathrm{~d} \theta \mathrm{d} s
\end{aligned}
$$

On the other hand, it holds that

$$
\begin{aligned}
& \int_{0}^{t} a_{s}\left(J_{\gamma} v, \psi_{n m}\right) \mathrm{d} s \\
& =\int_{0}^{t}\left\{a_{s}\left(\vartheta_{m}\left(J_{\gamma} v\right) * \varrho_{n}, \vartheta_{m}\left(J_{\gamma} v\right)_{t} * \varrho_{n}\right)+\gamma \vartheta_{m}^{2} a_{s}\left(\left(J_{\gamma} v\right) * \varrho_{n},\left(J_{\gamma} v\right) * \varrho_{n}\right)\right\} \mathrm{d} s \\
& \rightarrow \int_{0}^{t}\left\{\vartheta_{m}^{2} \gamma a_{s}\left(J_{\gamma} v, J_{\gamma} v\right)-\dot{\vartheta}_{m} \vartheta_{m} a_{s}\left(J_{\gamma} v, J_{\gamma} v\right)\right\} \mathrm{d} s .
\end{aligned}
$$

With regards to the nonlinear terms in the fluid component, we have as $n \rightarrow \infty$

$$
\int_{0}^{t} e^{\gamma s} B\left(u, u_{1}, \varphi_{n m}\right) \mathrm{d} s \rightarrow \int_{0}^{t} e^{\gamma s} \vartheta_{m}^{2} b\left(u, u_{1}, u\right) \mathrm{d} s .
$$

Using Corollary 4.4, one can see that

$$
\begin{aligned}
& \int_{0}^{t} e^{\gamma s} \vartheta_{m}^{2} b\left(u, u_{1}, u\right) \mathrm{d} s \\
& \leq C_{\varepsilon} e^{2 \gamma T} \int_{0}^{t}\|u\|_{\Omega_{f}}^{2}\left\|u_{1}\right\|_{V_{f}}^{2}\left(1+\left\|u_{1}\right\|_{\Omega_{f}}^{2 / 3}\right) \mathrm{d} s+\varepsilon \int_{0}^{t} a_{f}(u, u) \mathrm{d} s .
\end{aligned}
$$

As in [28, Chapter 1], if $t$ is a Lebesgue point of the integrand then

$$
\begin{aligned}
& -\int_{0}^{t} \dot{\vartheta}_{m} \vartheta_{m}\left\{\|u\|_{\Omega_{f}}^{2}+\|v\|_{\Omega_{s}}^{2}+a_{s}\left(J_{\gamma} v, J_{\gamma} v\right)\right\} \mathrm{d} s \\
& \rightarrow \frac{1}{2}\left(\|u(t)\|_{\Omega_{f}}^{2}+\|v(t)\|_{\Omega_{s}}^{2}+a_{s}\left(J_{\gamma} v(t), J_{\gamma} v(t)\right)\right) \text {, as } m \rightarrow \infty .
\end{aligned}
$$

Let $e(t)$ be the energy defined by (4.9) but without the indices $m$. Using the test functions $\left(\varphi_{n m}, \psi_{n m}\right)$ in (5.1), choosing $\varepsilon>0$ small enough, passing to the limit $n \rightarrow \infty$ and then 
$m \rightarrow \infty$ we have

$$
\begin{aligned}
& e(t)+\int_{0}^{t} a_{f}(u(\sigma), u(\sigma)) \mathrm{d} \sigma+\int_{0}^{t} a_{s}\left(J_{\gamma} v(\sigma), J_{\gamma} v(\sigma)\right)+\|v(\sigma)\|_{\Omega_{s}}^{2} \mathrm{~d} \sigma \\
& +\int_{0}^{t} e^{-2 \gamma \sigma}\left\|z^{r_{d}}(\sigma)\right\|_{\Omega_{s}}^{2} \mathrm{~d} \sigma+\int_{0}^{t} \int_{-r_{c}}^{0} e^{-2 \gamma \sigma}\|z(\sigma, \theta)\|_{\Omega_{s}}^{2} \mathrm{~d} \theta \mathrm{d} \sigma \\
& \leq C_{\gamma, T}\left(1+\left\|u_{1}\right\|_{L^{\infty}\left(0, T ; H_{f}\right)}^{2 / 3}\right) \int_{0}^{t}\|u\|_{\Omega_{f}}^{2}\left\|u_{1}\right\|_{V_{f}}^{2} \mathrm{~d} s
\end{aligned}
$$

for some constant $C_{\gamma, T}>0$. Since $u_{1} \in L^{2}\left(0, T ; V_{f}\right) \cap L^{\infty}\left(0, T ; H_{f}\right)$, one can apply Gronwall's inequality to obtain that $u$ is identically zero in $[0, t]$. This is true for almost every $t \in[0, T]$, hence it follows that (1.1) has only one weak solution.

We already knew from the existence part that $u$ and $z$ are continuous in time. Thus it remains to show continuity of the elastic component, and for this we derive a rescaledin-time energy identity. Given $\gamma>0$, let us define the functionals $\mathscr{E}: X \rightarrow \mathbb{R}$ and $\mathscr{D}$ : $V_{f} \times L^{2}\left(\Omega_{s}\right) \times L^{2}\left((-r, 0) \times \Omega_{s}\right) \times L^{2}\left(\Omega_{s}\right) \times L^{2}\left(\Omega_{s}\right) \rightarrow \mathbb{R}$ as follows

$$
\begin{aligned}
\mathscr{E}(u, w, v, z)= & \frac{1}{2}\left(\|u\|_{\Omega_{f}}^{2}+a_{s}(w, w)+\|v\|_{\Omega_{s}}^{2}\right) \\
& +\frac{1}{2} \int_{-r_{d}}^{0}\|z(\theta)\|_{\Omega_{s}}^{2} \mathrm{~d} \theta+\frac{1}{2} \int_{-r_{c}}^{0}\|z(\theta)\|_{\Omega_{s}}^{2} \mathrm{~d} \theta \\
\mathscr{D}(u, v, z, \zeta, \xi)= & a_{f}(u, u)+\gamma\|u\|_{\Omega_{f}}^{2}+\gamma a_{s}(w, w)+\left((\gamma+\mu-1) v+\mu_{d} \zeta, v\right)_{\Omega_{s}} \\
& +\int_{-r_{c}}^{0} \mu_{c}(\theta)(z(\theta), v)_{\Omega_{s}} \mathrm{~d} \theta+\frac{1}{2}\|\zeta\|_{\Omega_{s}}^{2}+\frac{1}{2}\|\xi\|_{\Omega_{s}}^{2} \\
& +\gamma \int_{-r_{d}}^{0}\|z(\theta)\|_{\Omega_{s}}^{2} \mathrm{~d} \theta+\gamma \int_{-r_{c}}^{0}\|z(\theta)\|_{\Omega_{s}}^{2} \mathrm{~d} \theta .
\end{aligned}
$$

By taking $\gamma>0$ sufficiently large enough, we can see that $\mathscr{D}$ is weakly lower semicontinuous. Indeed, using the elementary identity $(v, w)_{\Omega_{s}}=\frac{1}{2}\left(\|v+w\|_{\Omega_{s}}^{2}-\|v\|_{\Omega_{s}}^{2}-\|w\|_{\Omega_{s}}^{2}\right)$, the functional $\mathscr{D}$ can be rewritten as

$$
\begin{aligned}
\mathscr{D}(u, v, z, \zeta, \xi)=a_{f}(u, u)+\gamma\|u\|_{\Omega_{f}}^{2}+\gamma a_{s}(w, w)+\frac{1}{2}\left\|\zeta+\mu_{d} v\right\|_{\Omega_{s}}^{2}+\frac{1}{2}\|\xi\|_{\Omega_{s}}^{2} \\
+\left(\gamma+\mu-\frac{1}{2} \mu_{d}^{2}-\frac{1}{2}\left\|\mu_{c}\right\|_{L^{2}\left(-r_{c}, 0\right)}^{2}-1\right)\|v\|_{\Omega_{s}}^{2}+\gamma \int_{-r_{d}}^{0}\|z(\theta)\|_{\Omega_{s}}^{2} \mathrm{~d} \theta \\
\quad+\frac{1}{2} \int_{-r_{c}}^{0}\left\|z(\theta)+\mu_{c}(\theta) v\right\|_{\Omega_{s}}^{2} \mathrm{~d} \theta+\left(\gamma-\frac{1}{2}\right) \int_{-r_{c}}^{0}\|z(\theta)\|_{\Omega_{s}}^{2} \mathrm{~d} \theta .
\end{aligned}
$$

and weak lower semicontinuity follows immediately, provided that the coefficient of $\|v\|_{\Omega_{s}}^{2}$ is nonnegative.

Theorem 5.2. If $d=2$, then the weak solution of (1.1) constructed from the Faedo-Galerkin approximations satisfies the energy equation

$$
\begin{aligned}
& \mathscr{E}\left(u(t), w(t), w_{t}(t), z(t)\right)+\int_{0}^{t} e^{2 \gamma(t-\sigma)} \mathscr{D}\left(u(\sigma), w_{t}(\sigma), z(\sigma), z^{r_{d}}(\sigma), z^{r_{c}}(\sigma)\right) \mathrm{d} \sigma \\
& =e^{2 \gamma t} \mathscr{E}\left(u_{0}, w_{0}, v_{0}, z_{0}\right)+\int_{0}^{t} e^{2 \gamma(t-\sigma)}\left\{\left\langle G_{f}(\sigma), u(\sigma)\right\rangle_{V_{f}^{\prime} \times V_{f}}+\left(G_{s}(\sigma), w_{t}(\sigma)\right)_{\Omega_{s}}\right\} \mathrm{d} \sigma
\end{aligned}
$$

for almost every $t \in[0, T]$. 
Proof. We proceed using the methods in [28, Chapter 9]. For each nonnegative continuous function $\vartheta$ on $[0, T]$, the approximate solutions provided by the Faedo-Galerkin method give us the energy identity

$$
\begin{aligned}
& \int_{0}^{T} \mathscr{E}\left(u_{m}(t), w_{m}(t), v_{m}(t), e^{-\gamma t} z_{m}(t)\right) \vartheta(t) \mathrm{d} t \\
& +\int_{0}^{T} \int_{0}^{t} \mathscr{D}\left(u_{m}(\sigma), v_{m}(\sigma), e^{-\gamma \sigma} z_{m}(\sigma), e^{-\gamma \sigma} z_{m}^{r_{d}}(\sigma), e^{-\gamma \sigma} z_{m}^{r}(\sigma)\right) \vartheta(t) \mathrm{d} \sigma \mathrm{d} t \\
& =\int_{0}^{T} \mathscr{E}\left(u_{m 0}, w_{m 0}, v_{m 0}, z_{m 0}\right) \vartheta(t) \mathrm{d} t \\
& +\int_{0}^{T} \int_{0}^{t} e^{-\gamma \sigma}\left\{\left\langle G_{f}(\sigma), u_{m}(\sigma)\right\rangle_{V_{f}^{\prime} \times V_{f}}+\left(G_{s}(\sigma), v_{m}(\sigma)\right)_{\Omega_{s}}\right\} \vartheta(t) \mathrm{d} \sigma \mathrm{d} t .
\end{aligned}
$$

This can be obtained by choosing $\xi=r_{d}$ and $\xi=r_{c}$ in (4.7), taking the sum of these terms with (4.8), multipyling by $\vartheta$, integrating over the interval $[0, t]$ and then integrating over $[0, T]$.

According to the weak convergence of the approximate solutions and the weak lowersemicontinuity of $\mathscr{E}$, we have

$$
\begin{aligned}
& \liminf _{m \rightarrow \infty} \int_{0}^{T} \mathscr{E}\left(u_{m}(t), w_{m}(t), v_{m}(t), e^{-\gamma t} z_{m}(t)\right) \vartheta(t) \mathrm{d} t \\
& \geq \int_{0}^{T} e^{-2 \gamma t} \mathscr{E}\left(u(t), w(t), w_{t}(t), z(t)\right) \vartheta(t) \mathrm{d} t .
\end{aligned}
$$

Here, we recall that $\left(u_{\gamma}, v_{\gamma}, w_{\gamma}\right)=e^{-\gamma t}\left(u, w_{t}, w\right)$. On the other hand, the dominated convergence theorem, which is applicable due to (4.10), implies that

$$
\begin{aligned}
& \int_{0}^{T} \int_{0}^{t} e^{-\gamma \sigma}\left\{\left\langle G_{f}(\sigma), u_{m}(\sigma)\right\rangle_{V_{f}^{\prime} \times V_{f}}+\left(G_{s}(\sigma), v_{m}(\sigma)\right)_{\Omega_{s}}\right\} \vartheta(t) \mathrm{d} \sigma \mathrm{d} t \\
& \rightarrow \int_{0}^{T} \int_{0}^{t} e^{-2 \gamma \sigma}\left\{\left\langle G_{f}(\sigma), u(\sigma)\right\rangle_{V_{f}^{\prime} \times V_{f}}+\left(G_{s}(\sigma), w_{t}(\sigma)\right)_{\Omega_{s}}\right\} \vartheta(t) \mathrm{d} \sigma \mathrm{d} t .
\end{aligned}
$$

Using the weak convergence of $\left(u_{m}, w_{m t}, z_{m}, z_{m}^{r_{d}}, z_{m}^{r_{c}}\right)$ to $\left(e^{-\gamma t} u, e^{-\gamma t} w_{t}, z, z^{r_{d}}, z^{r_{c}}\right)$ in $L^{2}\left(0, T ; V_{f} \times L^{2}\left(\Omega_{s}\right) \times L^{2}\left(-r, 0 ; L^{2}\left(\Omega_{s}\right)\right) \times L^{2}\left(\Omega_{s}\right) \times L^{2}\left(\Omega_{s}\right)\right)$, Fatou's lemma and the weak lower-semicontinuity of $\mathscr{D}$, we can see that

$$
\begin{aligned}
& \liminf _{m \rightarrow \infty} \int_{0}^{T} \int_{0}^{t} \mathscr{D}\left(u_{m}(\sigma), v_{m}(\sigma), e^{-\gamma \sigma} z_{m}(\sigma), e^{-\gamma \sigma} z_{m}^{r_{d}}(\sigma), e^{-\gamma \sigma} z_{m}^{r_{c}}(\sigma)\right) \vartheta(t) \mathrm{d} \sigma \mathrm{d} t \\
& \geq \int_{0}^{T} \int_{0}^{t} e^{-2 \gamma \sigma} \mathscr{D}\left(u(\sigma), w_{t}(\sigma), z(\sigma), z^{r_{d}}(\sigma), z^{r_{c}}(\sigma)\right) \vartheta(t) \mathrm{d} \sigma \mathrm{d} t .
\end{aligned}
$$

Similarly, from the strong convergence of the approximate initial data, there holds

$$
\int_{0}^{T} \mathscr{E}\left(u_{m 0}, w_{m 0}, v_{m 0}, z_{m 0}\right) \vartheta(t) \mathrm{d} t \rightarrow \int_{0}^{T} \mathscr{E}\left(u_{0}, w_{0}, v_{0}, z_{0}\right) \vartheta(t) \mathrm{d} t .
$$

Since $\vartheta \geq 0$ is arbitrary, we obtain that the left hand side of (5.2) is smaller that its right hand side.

To obtain the reverse inequality, we use the same argument as in the uniqueness part to prove that the energy identity (5.2) holds where the lower limit 0 of integration is replaced 
by an arbitrary $s \in(0, t)$. According to the weak continuity of solutions,

$$
\liminf _{s \downarrow 0} \mathscr{E}\left(u(s), w(s), w_{t}(s), z(s)\right) \geq \mathscr{E}\left(u_{0}, w_{0}, v_{0}, z_{0}\right) .
$$

Passing to the limit inferior of the said energy identity yields the reverse inequality.

From the above energy identity, we obtain the continuity of weak solutions.

Corollary 5.3. If $d=2$, then the weak solution constructed from the Faedo-Galerkin approximations satisfies $\left(u, w, w_{t}, z\right) \in C(0, T ; X)$.

Proof. We already know that $z \in C\left(0, T ; L^{2}\left((-r, 0) \times \Omega_{s}\right)\right)$ and since $d=2$ it holds that $u \in C\left(0, T ; H_{f}\right)$. Hence, it remains to establish the continuity of the structure displacement and velocity. Denote by $\mathscr{E}_{w}$ the component of the energy with respect to the elastic body, that is, $\mathscr{E}_{w}(t)=\left\|w_{t}(t)\right\|_{\Omega_{s}}^{2}+a_{s}(w(t), w(t))$. Consider the energy identity at times $t$ and $s$ for $0 \leq s \leq t \leq T$ and subtract the two resulting equations. Doing so we obtain that $\left|\mathscr{E}_{w}(t)-\mathscr{E}_{w}(s)\right| \leq \int_{s}^{t} h(\sigma) \mathrm{d} \sigma+o(t-s)$ as $s \rightarrow t$ for some $h \in L^{1}(0, T)$. The absolutely continuity of the Lebesgue integral implies that for a given $\varepsilon>0$ there is $\delta>0$ such that $\left|\mathscr{E}_{w}(t)-\mathscr{E}_{w}(s)\right|<\varepsilon+o(t-s)$ whenever $|t-s|<\delta$. Note that

$$
\begin{aligned}
& \left\|w_{t}(t)-w_{t}(s)\right\|_{\Omega_{s}}^{2}+a_{s}(w(t)-w(s), w(t)-w(s)) \\
& =\mathscr{E}_{w}(t)+\mathscr{E}_{w}(s)-2\left(w_{t}(t), w_{t}(s)\right)_{\Omega_{s}}-2 a_{s}(w(t), w(s)) .
\end{aligned}
$$

According to weak continuity, we have $\left(w_{t}(t), w_{t}(s)\right)_{\Omega_{s}}+a_{s}(w(t), w(s)) \rightarrow \mathscr{E}_{w}(t)$ as $s \rightarrow t$. Applying this to (5.3), we obtain $w_{t} \in C\left(0, T ; L^{2}\left(\Omega_{s}\right)\right)$ and $w \in C\left(0, T ; H^{1}\left(\Omega_{s}\right)\right)$.

\section{Strong Solutions in Two Dimensions}

The present section is devoted to establishing that the weak solution constructed in Section 4 is a strong one provided that the data are smooth enough and satisfy suitable compatibility conditions. We limit our study to the case of two dimensions. We consider the following assumption:

(A) Suppose that $\left(u_{0}, w_{0}, v_{0}, z_{0}\right) \in\left(V_{f} \cap H^{2}\left(\Omega_{f}\right)\right) \times H^{2}\left(\Omega_{s}\right) \times H^{1}\left(\Omega_{s}\right) \times H^{1}\left((-r, 0) \times \Omega_{s}\right)$ satisfies $\left.u_{0}\right|_{\Gamma_{s}}=\left.v_{0}\right|_{\Gamma_{s}}$ and $\left.z_{0}\right|_{\theta=0}=v_{0}$. Moreover, there exists $p_{0} \in H^{1}\left(\Omega_{f}\right)$ such that the following compatibility condition holds

$$
\sigma\left(w_{0}\right) \cdot \nu=\frac{\partial u_{0}}{\partial \nu}-p_{0} \nu+\frac{1}{2}\left(u_{0} \cdot \nu\right) u_{0} \text { on } \Gamma_{s} .
$$

The compatibility conditions $\left.u_{0}\right|_{\Gamma_{s}}=\left.v_{0}\right|_{\Gamma_{s}}$ and (6.1) are adapted from [16] in the linear case without delay. A similar condition is given in $[\mathbf{1 0}]$ for the nonlinear case. The compatibility condition $\left.z_{0}\right|_{\theta=0}=v_{0}$ is a typical assumption for the regularity of solutions for delay differential equations. This means that the trace of the initial history at $\theta=0$ must fit with the initial structure velocity.

In the proof we use symbolic calculus. Let $A$ and $B$ be pseudo-differential operators in $\mathbb{R}^{d}$ with symbols $a$ and $b$ having orders $m$ and $n$, respectively. The symbol of the commutator $[A, B]=A B-B A$ is given by the Poisson bracket

$$
\{a, b\}:=\frac{1}{i} \sum_{j=1}^{d}\left(\frac{\partial a}{\partial x_{j}} \frac{\partial b}{\partial \xi_{j}}-\frac{\partial a}{\partial \xi_{j}} \frac{\partial b}{\partial x_{j}}\right)
$$

modulo a symbol with order $m+n-2$. Here $\xi$ denotes the Fourier symbol corresponding to spatial variable $x$. Thus $[A, B]$ is an operator of order $m+n-1$. For more details on pseudo-differential operators and commutators, we refer the reader to [18, Chapter 2]. 
For the proof of the regularity result, we follow the arguments presented in [10], with few modifications. We summarize the steps as follows:

1. Reformulate the Faedo-Galerkin approximations using the method in [16] applied to the linearized case.

2. Prove boundedness for the time derivatives of the state variables using the regularity and compatibility conditions of the initial data and history.

3. Establish preliminary regularity of the nonlinear terms $(u \cdot \nabla u)$ and $(u \cdot \nu) u$.

4. Show the existence of a pressure satisfying the fluid equation and the stress boundary condition on the interface.

5. Prove interior regularity of the structure displacement by localization away from the interface, and utilize commutator estimates and regularity theory for steady elasticity equations with homogeneous Dirichlet boundary conditions.

6. Prove interior regularity of the fluid velocity by using the strategy in step 5 twice, now appealing to regularity of the Stokes equations.

7. Localize the problem by considering a partition of unity subordinate to an open cover of the interface and apply Melrose-Sjöstrand coordinates to transform the equations on a half-space, for which the velocity in general is not divergence-free.

8. Establish additional regularity of the transformed state variables in the tangential direction by the energy method.

9. Invoke Sobolev embedding to show $L^{2}$-regularity of the nonlinear convection term.

10. Prove $H^{2}$-regularity in space for the fluid velocity and pressure utilizing the equations governing the fluid.

11. Prove $H^{2}$-regularity in space for structure displacement by using the elasticity equation and regularity theory for elliptic problems with Dirichlet boundary conditions.

Step 1 is a technical strategy that has been considered in [16] in the linear case without delay. We would like to emphasize that this is introduced in order to derive strong a priori estimates with respect to the norms in $V_{f}$ and $H^{1}\left(\Omega_{s}\right)$ of the fluid and structure velocities, respectively.

Theorem 6.1. Let $d=2$. Assume that hypothesis (A) is satisfied, and suppose that $G_{f} \in$ $L^{2}\left(0, T ; L^{2}\left(\Omega_{f}\right)\right) \cap H^{1}\left(0, T ; V_{f}^{\prime}\right)$ with $G_{f}(0) \in L^{2}\left(\Omega_{f}\right)$ and $G_{s} \in H^{1}\left((0, T) \times \Omega_{s}\right)$. Then the weak solution of (1.1)-(1.2) satisfies the following regularity properties:

(a) $u \in L^{2}\left(0, T ; V_{f} \cap H^{2}\left(\Omega_{f}\right)\right), u_{t} \in L^{\infty}\left(0, T ; H_{f}\right) \cap L^{2}\left(0, T ; V_{f}\right)$

(b) $w \in L^{2}\left(0, T ; H^{2}\left(\Omega_{s}\right)\right), w_{t} \in L^{\infty}\left(0, T ; H^{1}\left(\Omega_{s}\right)\right), w_{t t} \in L^{\infty}\left(0, T ; L^{2}\left(\Omega_{s}\right)\right)$

(c) $z \in C\left(0, T ; H^{1}\left((-r, 0) \times \Omega_{s}\right)\right), z_{t} \in C\left(0, T ; L^{2}\left((-r, 0) \times \Omega_{s}\right)\right)$, $z^{\xi} \in H^{1}\left((0, T) \times \Omega_{s}\right)$ for every $\xi \in[0, r]$.

Moreover, there exists $p \in L^{2}\left(0, T ; H^{1}\left(\Omega_{f}\right)\right)$ such that the strong form of the equations are satisfied almost everywhere.

Proof. For simplicity of exposition, we shall assume that $\mu>0$ is large enough. This is not a restriction since one can proceed by a time-rescaling argument as in the proof of the existence part. The proof is divided into several steps. The first part is to consider a different formulation of the Galerkin approximations. Afterwards, we prove additional timeregularity and then spatial regularity following [10] along with Agmon-Douglis-Nirenberg type arguments in $[\mathbf{1}]$. 
Step 1. Reformulation of Faedo-Galerkin approximations. Following [16], we reconsider the (non-rescaled) Faedo-Galerkin step using the approximate system

$$
\begin{aligned}
& \frac{d}{d t}\left(u_{m}(t), \varphi\right)_{\Omega_{f}}+a_{f}\left(u_{m}(t), \varphi\right)+b\left(u_{m}(t), u_{m}(t), \varphi\right)+\frac{d}{d t}\left(v_{m}(t), \psi\right)_{\Omega_{s}} \\
& \quad+a_{s}\left(J v_{m}(t), \psi\right)+\left(\mu v_{m}(t)+\mu_{d} z_{m}^{r_{d}}(t), \psi\right)_{\Omega_{s}}+\int_{-r_{c}}^{0} \mu_{c}(\theta)\left(z_{m}(t, \theta), \psi\right)_{\Omega_{s}} \mathrm{~d} \theta \\
& =\left(G_{f}(t), \varphi\right)_{\Omega_{f}}+\left(G_{s}(t), \psi\right)_{\Omega_{s}}-a_{s}\left(w_{0}, \psi\right)+a_{f}\left(u_{m}(0)-u_{0}, \varphi\right) \\
& \quad+b\left(u_{m}(0)-u_{0}, u_{m}(0), \varphi\right)+b\left(u_{0}, u_{m}(0)-u_{0}, \varphi\right)
\end{aligned}
$$

for every $(\varphi, \psi)$ in the linear span of $\left\{\left(\varphi_{j}, \psi_{j}\right)\right\}_{j=1}^{m}$, together with the initial conditions $u_{m}(0)=\left.\Pi_{m}\left(u_{0}, v_{0}\right)\right|_{\Omega_{f}}, v_{m}(0)=\left.\Pi_{m}\left(u_{0}, v_{0}\right)\right|_{\Omega_{s}}$ and $z_{m}(0, \theta)=\left.\Pi_{m}\left(u_{0}, z_{0}(\theta)\right)\right|_{\Omega_{s}}=: z_{m 0}(\theta)$, where $\Pi_{m}$ is the projection operator defined in (2.7).

Let us analyze the additional terms on the right hand side for this new type of approximations. First, we have the following estimates

$$
\begin{aligned}
\left|a_{f}\left(u_{m}(0)-u_{0}, \varphi\right)\right| & \leq \varepsilon\|\varphi\|_{V_{f}}^{2}+C_{\varepsilon}\left\|u_{m}(0)-u_{0}\right\|_{V_{f}}^{2} \\
& \leq \varepsilon\|\varphi\|_{V_{f}}^{2}+C_{\varepsilon}\left\|\left(u_{0}, v_{0}\right)\right\|_{H_{1}}^{2} \\
\left|b\left(u_{m}(0)-u_{0}, u_{m}(0), \varphi\right)\right| & \leq C\left\|u_{m}(0)-u_{0}\right\|_{V_{f}}\left\|u_{m}(0)\right\|_{V_{f}}\|\varphi\|_{V_{f}} \\
& \leq \varepsilon\|\varphi\|_{V_{f}}^{2}+C_{\varepsilon}\left\|\left(u_{0}, v_{0}\right)\right\|_{H_{1}}^{4} \\
\left|b\left(u_{m}(0), u_{m}(0)-u_{0}, \varphi\right)\right| & \leq \varepsilon\|\varphi\|_{V_{f}}^{2}+C_{\varepsilon}\left\|\left(u_{0}, v_{0}\right)\right\|_{H_{1}}^{4} .
\end{aligned}
$$

From these estimates, and using the same argument as in Theorem 4.5, we obtain a weak solution to (1.1)-(1.2). By uniqueness, this is the same as the one constructed from Theorem 4.5 .

Step 2. Time regularity. In this step, we show that $\left(u_{t}, w_{t}, w_{t t}, z_{t}\right) \in L^{\infty}(0, T ; X)$ and $u_{t} \in L^{2}\left(0, T ; V_{f}\right)$. According to $\left.z_{0}\right|_{\theta=0}=v_{0}$, we have the compatibility condition $z_{m 0}(0)=$ $\alpha_{m 0}$, and as a consequence $h_{m} \in C\left(0, T ; H^{1}(-r, 0)\right) \cap C^{1}\left(0, T ; L^{2}(-r, 0)\right), \alpha_{m}, f_{m} \in H^{2}(0, T)$ and $h_{m}^{\xi} \in H^{1}(0, T)$ for every $\xi \in[0, r]$. Taking the time-derivative of (6.2) and setting $\varphi=\partial_{t} u_{m}(t)$ and $\psi=\partial_{t} v_{m}(t)$ yields

$$
\begin{aligned}
& \frac{1}{2} \frac{d}{d t}\left\{\left\|\partial_{t} u_{m}(t)\right\|_{\Omega_{f}}^{2}+\left\|\partial_{t} v_{m}(t)\right\|_{\Omega_{s}}^{2}+a_{s}\left(v_{m}(t), v_{m}(t)\right)\right\}+a_{f}\left(\partial_{t} u_{m}(t), \partial_{t} u_{m}(t)\right) \\
& +\left(\mu \partial_{t} v_{m}(t)+\mu_{d} \partial_{t} z_{m}^{r_{d}}(t), \partial_{t} v_{m}(t)\right)_{\Omega_{s}}+\int_{-r_{c}}^{0} \mu_{c}(\theta)\left(\partial_{t} z_{m}(t, \theta), \partial_{t} v_{m}(t)\right)_{\Omega_{s}} \mathrm{~d} \theta \\
& =\left(\partial_{t} G_{f}(t), \partial_{t} u_{m}(t)\right)_{\Omega_{f}}+\left(\partial_{t} G_{s}(t), \partial_{t} v_{m}(t)\right)_{\Omega_{s}}-b\left(\partial_{t} u_{m}(t), u_{m}(t), \partial_{t} u_{m}(t)\right) .
\end{aligned}
$$

For the trilinear term, we apply Corollary 4.4 to obtain

$$
\begin{aligned}
& \left|b\left(\partial_{t} u_{m}(t), u_{m}(t), \partial_{t} u_{m}(t)\right)\right| \\
& \leq \varepsilon\left\|\partial_{t} u_{m}(t)\right\|_{V_{f}}^{2}+C_{\varepsilon}\left\|\partial_{t} u_{m}(t)\right\|_{\Omega_{f}}^{2}\left\|u_{m}(t)\right\|_{V_{f}}^{2}\left(1+\left\|u_{m}(t)\right\|_{\Omega_{f}}^{2 / 3}\right) .
\end{aligned}
$$

Let $Y_{m}(t)=\mathscr{E}\left(\partial_{t} u_{m}(t), v_{m}(t), \partial_{t} v_{m}(t), \partial_{t} z_{m}(t)\right)$. Using (6.4) in (6.3) and then applying Gronwall's inequality, we have

$$
\begin{aligned}
& Y_{m}(t) \leq C Y_{m}(0)+C \int_{0}^{t}\left\|\partial_{t} G_{f}(\sigma)\right\|_{V_{f}^{\prime}}^{2}+\left\|\partial_{t} G_{s}(\sigma)\right\|_{\Omega_{s}}^{2} \mathrm{~d} s \\
& +C \int_{0}^{t}\left\|\partial_{t} u_{m}(\sigma)\right\|_{\Omega_{f}}^{2}\left(1+\left\|u_{m}(\sigma)\right\|_{V_{f}}^{2}\right)\left(1+\left\|u_{m}(\sigma)\right\|_{\Omega_{f}}^{2 / 3}\right) \mathrm{d} s .
\end{aligned}
$$


From this estimate, we have the boundedness of $Y_{m}(t)$ once we have shown the boundedness of $Y_{m}(0)$. First note that $\partial_{t} z_{m}(0)=\partial_{\theta} z_{m 0}$, and so $\left\|\partial_{t} z_{m}(0)\right\|_{L^{2}\left(-r, 0 ; L^{2}\left(\Omega_{s}\right)\right)} \leq$ $\left\|z_{0}\right\|_{L^{2}\left(-r, 0 ; H^{1}\left(\Omega_{s}\right)\right)}$. Also, $\left|a_{s}\left(v_{m}(0), v_{m}(0)\right)\right| \leq C\left\|\left(u_{0}, v_{0}\right)\right\|_{H_{1}}$ by Korn's inequality and the fact that the projections $\Pi_{m}$ for $m \in \mathbb{N}$ are uniformly bounded with respect to the norm in $H_{1}$. Setting $t=0$ in the reformulated approximate system (6.2) in Step 1, and taking $(\varphi, \psi)=\left(\partial_{t} u_{m}(0), \partial_{t} v_{m}(0)\right)$ as the test functions, one obtains

$$
\begin{aligned}
& \left\|\partial_{t} u_{m}(0)\right\|_{\Omega_{f}}^{2}+\left\|\partial_{t} v_{m}(0)\right\|_{\Omega_{s}}^{2}+a_{f}\left(u_{0}, \partial_{t} u_{m}(0)\right)+b\left(u_{0}, u_{0}, \partial_{t} u_{m}(0)\right) \\
& +a_{s}\left(w_{0}, \partial_{t} v_{m}(0)\right)+\left(\mu v_{m}(0)+\mu_{d} z_{m}^{r_{d}}(0), \partial_{t} v_{m}(0)\right)_{\Omega_{s}} \\
& +\int_{-r_{c}}^{0} \mu_{c}(\theta)\left(z_{m}(0, \theta), \partial_{t} v_{m}(0)\right)_{\Omega_{s}} \mathrm{~d} \theta=\left(G_{f}(0), \partial_{t} u_{m}(0)\right)_{\Omega_{f}}+\left(G_{s}(0), \partial_{t} v_{m}(0)\right)_{\Omega_{s}} .
\end{aligned}
$$

Again, utilizing the uniform boundedness of $\Pi_{m}$ in $H_{1}$, we have

$$
\begin{aligned}
& \left|\left(G_{f}(0), \partial_{t} u_{m}(0)\right)_{\Omega_{f}}\right| \leq \varepsilon\left\|\partial_{t} u_{m}(0)\right\|_{\Omega_{f}}^{2}+C_{\varepsilon}\left\|G_{f}(0)\right\|_{L^{2}\left(\Omega_{f}\right)}^{2} \\
& \left|\left(G_{s}(0), \partial_{t} v_{m}(0)\right)_{\Omega_{s}}\right| \leq \varepsilon\left\|\partial_{t} v_{m}(0)\right\|_{\Omega_{s}}^{2}+C_{\varepsilon}\left\|G_{s}\right\|_{H^{1}\left(0, T ; L^{2}\left(\Omega_{s}\right)\right)}^{2} \\
& \left|\left(\mu v_{m}(0)+\mu_{d} z_{m}^{r_{d}}(0), \partial_{t} v_{m}(0)\right)_{\Omega_{s}}\right|+\int_{-r_{c}}^{0}\left|\mu_{c}(\theta)\left(z_{m}(0, \theta), \partial_{t} v_{m}(0)\right)_{\Omega_{s}}\right| \mathrm{d} \theta \\
& \quad \leq \varepsilon\left\|\partial_{t} v_{m}(0)\right\|_{\Omega_{s}}^{2}+C_{\varepsilon}\left\{\left\|\left(u_{0}, v_{0}\right)\right\|_{H_{1}}^{2}+\left\|z_{0}\right\|_{H^{1}\left((-r, 0) \times \Omega_{s}\right)}^{2}\right\} .
\end{aligned}
$$

Furthermore, the divergence theorem and condition (A) imply that

$$
\begin{aligned}
& a_{f}\left(u_{0}, \partial_{t} u_{m}(0)\right)+b\left(u_{0}, u_{0}, \partial_{t} u_{m}(0)\right)+a_{s}\left(w_{0}, \partial_{t} v_{m}(0)\right) \\
& =-\int_{\Omega_{f}} \Delta u_{0} \cdot \partial_{t} u_{m}(0) \mathrm{d} x-\int_{\Omega_{s}} \operatorname{div} \sigma\left(w_{0}\right) \cdot \partial_{t} v_{m}(0)+w_{0} \cdot \partial_{t} v_{m}(0) \mathrm{d} x \\
& +\int_{\Omega_{f}}\left(u_{0} \cdot \nabla\right) u_{0} \cdot \partial_{t} u_{m}(0) \mathrm{d} x+\int_{\Gamma_{s}}\left(\sigma\left(w_{0}\right) \cdot \nu-\frac{\partial u_{0}}{\partial \nu}-\frac{1}{2}\left(u_{0} \cdot \nu\right) u_{0}\right) \cdot \partial_{t} u_{m}(0) \mathrm{d} x \\
& \leq \varepsilon\left\|\partial_{t} u_{m}(0)\right\|_{\Omega_{f}}^{2}+\varepsilon\left\|\partial_{t} v_{m}(0)\right\|_{\Omega_{s}}^{2}+C_{\varepsilon}\left\{\left\|u_{0}\right\|_{H^{2}\left(\Omega_{s}\right)}^{2}+\left\|w_{0}\right\|_{H^{2}\left(\Omega_{s}\right)}^{2}+\left\|p_{0}\right\|_{H^{1}\left(\Omega_{s}\right)}^{2}\right\}
\end{aligned}
$$

Using (6.7)-(6.10) in (6.6), we see that $Y_{m}(0)$ is uniformly bounded with respect to $m$. As a consequence, $\left(u_{t}, w_{t}, w_{t t}, z_{t}\right) \in L^{\infty}(0, T ; X)$ from (6.5). In particular $w_{t} \in H^{1}\left((0, T) \times \Omega_{s}\right)$ and from the compatibility conditions we obtain the regularity properties of $z$ stated in (c) according to Theorem 3.3. Moreover, we have $u_{t} \in L^{2}\left(0, T ; V_{f}\right)$, hence $u \in C\left(0, T ; V_{f}\right)$.

Step 3. Preliminary regularity of nonlinear terms. For this part, we prove that $(u \cdot \nabla) u \in$ $L^{2}\left(0, T ; L^{\frac{3}{2}}\left(\Omega_{f}\right)\right)$ and $(u \cdot \nu) u \in L^{2}\left(0, T ; L^{2}\left(\Gamma_{s}\right)\right)$. Take $\varphi \in L^{2}\left(0, T ; H^{\frac{1}{2}}\left(\Gamma_{s}\right)\right)$. Using trace theorem, Poincaré inequality and the embedding $H^{\frac{1}{2}}\left(\Gamma_{s}\right) \subset L^{4}\left(\Gamma_{s}\right)$, we have

$$
\begin{aligned}
& \int_{0}^{T}\langle(u(t) \cdot \nu) u(t), \varphi(t)\rangle \mathrm{d} t \leq \int_{0}^{T}\|u(t)\|_{L^{4}\left(\Gamma_{s}\right)}^{2}\|\varphi(s)\|_{L^{2}\left(\Gamma_{s}\right)} \mathrm{d} t \\
& \leq C \int_{0}^{T}\|u(t)\|_{V_{f}}^{2}\|\varphi(t)\|_{L^{2}\left(\Gamma_{s}\right)} \mathrm{d} t \leq C \sqrt{T}\|u\|_{L^{\infty}\left(0, T ; V_{f}\right)}^{2}\|\varphi\|_{L^{2}\left(0, T ; L^{2}\left(\Gamma_{s}\right)\right)} .
\end{aligned}
$$

This shows that $(u \cdot \nu) u \in L^{2}\left(0, T ; L^{2}\left(\Gamma_{s}\right)\right)$. Let $\phi \in L^{2}\left(0, T ; L^{3}\left(\Omega_{f}\right)\right)$. In virtue of Hölder's inequality and the embedding $V_{f} \subset L^{6}\left(\Omega_{f}\right)$, we obtain

$$
\begin{aligned}
& \int_{0}^{T} \int_{\Omega_{f}}(u(t) \cdot \nabla) u(t) \cdot \varphi(t) \mathrm{d} x \mathrm{~d} t \leq \int_{0}^{T}\|u(t)\|_{L^{6}\left(\Omega_{f}\right)}\|\nabla u(s)\|_{L^{2}\left(\Omega_{f}\right)}\|\phi(s)\|_{L^{3}\left(\Omega_{f}\right)} \mathrm{d} x \\
& \leq C \int_{0}^{T}\|u(s)\|_{V_{f}}^{2}\|\varphi(s)\|_{L^{3}\left(\Omega_{f}\right)} \mathrm{d} x \leq C \sqrt{T}\|u\|_{L^{\infty}\left(0, T ; V_{f}\right)}^{2}\|\phi\|_{L^{2}\left(0, T ; L^{3}\left(\Omega_{f}\right)\right)} .
\end{aligned}
$$


Thus $(u \cdot \nabla) u \in L^{2}\left(0, T ; L^{\frac{3}{2}}\left(\Omega_{f}\right)\right)$ by duality.

Step 4. Existence of pressure term. We prove that there is $p \in L^{2}\left(0, T ; L^{2}\left(\Omega_{f}\right)\right)$ with

$$
\begin{aligned}
& \operatorname{div}(\nabla u-p I) \in L^{2}\left(0, T ; L^{\frac{3}{2}}\left(\Omega_{f}\right)\right), \\
& (\nabla u-p I) \cdot \nu \in L^{2}\left(0, T ; H^{-\frac{1}{2}}\left(\Gamma_{s}\right)\right) .
\end{aligned}
$$

Choosing $\varphi \in H^{1}\left(0, T ; H_{0}^{1}\left(\Omega_{f}\right) \cap V_{f}\right)$ with $\left.\varphi\right|_{t=T}=0$ and $\psi=0$ in the weak formulation Definition 4.1(e), we have

$$
\int_{0}^{T}\left(u_{t}-\Delta u+(u \cdot \nabla) u-G_{f}, \varphi\right)_{\Omega_{f}} \mathrm{~d} t=0 .
$$

By density, this holds for every $\varphi \in L^{2}\left(0, T ; H_{0}^{1}\left(\Omega_{f}\right) \cap V_{f}\right)$. According to de Rham's Theorem, see for example [41, Lemma 1.4.1, Sect. IV.1.4], there exists $\pi \in L^{2}\left(0, T ; L^{2}\left(\Omega_{f}\right)\right)$ such that in the sense of distributions

$$
u_{t}-\Delta u+(u \cdot \nabla) u+\nabla \pi=G_{f} .
$$

Since $u_{t},(u \cdot \nabla u) \in L^{2}\left(0, T ; L^{\frac{3}{2}}\left(\Omega_{f}\right)\right)$ we have $\operatorname{div}(\nabla u-\pi I) \in L^{2}\left(0, T ; L^{\frac{3}{2}}\left(\Omega_{f}\right)\right)$ and therefore $(\nabla u-p I) \cdot \nu \in L^{2}\left(0, T ; W^{-\frac{2}{3}, \frac{3}{2}}\left(\Gamma_{s}\right)\right)$ according to [35, Chapter 2, Theorem 5.7]. Observe that $p=\pi+\pi^{*}$ still satisfies equation (6.11) for any $\pi^{*} \in L^{2}(0, T)$. We shall pick a specific $\pi^{*}$ below.

By taking $\varphi=0$ and $\psi \in H^{1}\left(0, T ; H_{0}^{1}\left(\Omega_{s}\right)\right)$ with $\left.\psi\right|_{t=T}=0$ in the weak formulation, we see that

$$
-\operatorname{div} \sigma(w)=G_{s}-w_{t t}-w-\mu w_{t}-\mu_{d} z^{r_{d}}-\int_{r_{c}}^{0} \mu_{c} z \mathrm{~d} \theta
$$

holds in the sense of distributions. Since the right hand side of the above equation lies in $L^{2}\left(0, T ; L^{2}\left(\Omega_{s}\right)\right)$, we also have $\operatorname{div} \sigma(w) \in L^{2}\left(0, T ; L^{2}\left(\Omega_{s}\right)\right)$. Thus $\sigma(w) \cdot \nu \in L^{2}\left(0, T ; H^{-\frac{1}{2}}\left(\Gamma_{s}\right)\right)$. From the embedding $H^{-\frac{1}{2}}\left(\Gamma_{s}\right) \subset W^{\frac{2}{3}, 3}\left(\Gamma_{s}\right)^{\prime}=W^{-\frac{2}{3}, \frac{3}{2}}\left(\Gamma_{s}\right)$, it follows that we have $\sigma(w) \cdot \nu \in$ $L^{2}\left(0, T ; W^{-\frac{2}{3}, \frac{3}{2}}\left(\Gamma_{s}\right)\right)$.

Let $\phi \in L^{2}\left(0, T ; W^{\frac{2}{3}, 3}\left(\Gamma_{s}\right)\right)$. By trace theory there exists $\psi \in L^{2}\left(0, T ; W^{1,3}\left(\Omega_{s}\right)\right)$ such that $\psi=\phi$ on $\Gamma_{s}$. We decompose $\phi=(\phi-\kappa \nu)+\kappa \nu$, where $\kappa=\frac{1}{\left|\Gamma_{s}\right|} \int_{\Gamma_{s}} \phi \cdot \nu \mathrm{d} x$ so that $\int_{\Gamma_{s}}(\phi-\kappa \nu) \cdot \nu \mathrm{d} x=0$. Let $(\varphi, \varpi) \in L^{2}\left(0, T ; W^{1,3}\left(\Omega_{f}\right)\right) \times L^{2}\left(0, T ; L^{3}\left(\Omega_{f}\right) / \mathbb{R}\right)$ be the solution of the time-dependent Stokes problem

$$
\begin{cases}-\Delta \varphi+\nabla \varpi=0, & \text { in }(0, T) \times \Omega_{f}, \\ \operatorname{div} \varphi=0, & \text { in }(0, T) \times \Omega_{f}, \\ u=0, & \text { on }(0, T) \times \Gamma_{f}, \\ u=\phi-\kappa \nu, & \text { on }(0, T) \times \Gamma_{s} .\end{cases}
$$

Then we have $(\varphi, \psi) \in L^{2}\left(0, T ; W^{1,3}\left(\Omega_{f}\right) \times W^{1,3}\left(\Omega_{s}\right)\right)$. Let us choose the function $\pi^{*} \in$ $L^{2}(0, T)$ according to

$$
\pi^{*}(t)=\frac{1}{\left|\Gamma_{s}\right|}\left\langle(\nabla u(t)-\pi(t)) \cdot \nu+\frac{1}{2}(u(t) \cdot \nu) \cdot u(t)-\sigma(w(t)) \cdot \nu, \nu\right\rangle_{W^{-\frac{2}{3}, \frac{3}{2}}\left(\Gamma_{s}\right) \times W^{\frac{2}{3}, 3}\left(\Gamma_{s}\right)} .
$$

Compare this with $(2.4)$ in the linear static case. By taking $(\varphi, \psi)$ as the test function in Definition 4.1(e) and applying the generalized Green's identities both in the fluid and solid domains we have, following computations in the proof of Theorem 2.2,

$$
(\nabla u-p I) \cdot \nu+\frac{1}{2}(u \cdot \nu) \cdot u-\sigma(w) \cdot \nu=0 \text { in } L^{2}\left(0, T ; W^{-\frac{2}{3}, \frac{3}{2}}\left(\Gamma_{s}\right)\right) .
$$


Since $\sigma(w) \cdot \nu \in L^{2}\left(0, T ; H^{-\frac{1}{2}}\left(\Gamma_{s}\right)\right)$ and $(u \cdot \nu) \cdot u \in L^{2}\left(0, T ; L^{2}\left(\Gamma_{s}\right)\right)$, we have

$$
(\nabla u-p I) \cdot \nu \in L^{2}\left(0, T ; H^{-\frac{1}{2}}\left(\Gamma_{s}\right)\right) .
$$

Collecting what we have obtained so far, the following equations holds for almost every point in the space-time domain

$$
\begin{cases}u_{t}-\Delta u+(u \cdot \nabla) u+\nabla p=G_{f}, & \text { in } L^{2}\left(0, T ; L^{\frac{3}{2}}\left(\Omega_{f}\right)\right), \\ \operatorname{div} u=0, & \text { in } L^{2}\left(0, T ; L^{2}\left(\Omega_{f}\right)\right), \\ u=0, & \text { in } L^{2}\left(0, T ; H^{\frac{1}{2}}\left(\Gamma_{f}\right)\right), \\ u=w_{t}, & \text { in } L^{2}\left(0, T ; H^{\frac{1}{2}}\left(\Gamma_{s}\right)\right), \\ w_{t t}-\operatorname{div} \sigma(w)+w+\mu w_{t}+\mu_{d} z^{r_{d}}+\int_{-r_{c}}^{0} \mu_{c} z \mathrm{~d} \theta=G_{s}, & \text { in } L^{2}\left(0, T ; L^{2}\left(\Omega_{s}\right)\right), \\ \sigma(w) \cdot \nu=\frac{\partial u}{\partial \nu}-p \nu+\frac{1}{2}(u \cdot \nu) u, & \text { in } L^{2}\left(0, T ; H^{-\frac{1}{2}}\left(\Gamma_{s}\right)\right) .\end{cases}
$$

Step 5. Interior regularity of the solid component. Given $\delta>0$, we define $\Omega_{s}^{\delta}:=\{x \in$ $\left.\Omega_{s}: \operatorname{dist}\left(x, \Gamma_{s}\right)>\delta\right\}$. Suppose that $\chi_{1} \in C_{0}^{\infty}\left(\Omega_{s}\right)$ is a cut-off function such that $\chi_{1}=1$ on $\Omega_{s}^{\delta}$. Multiplying the elastic equation by this function, we have the elliptic boundary value problem

$$
\begin{cases}-\operatorname{div} \sigma\left(\chi_{1} w\right)+\left(\chi_{1} w\right)=\chi_{1} F-\left[\operatorname{div} \sigma, \chi_{1}\right] w, & \text { in }(0, T) \times \Omega_{s} \\ \chi_{1} w=0, & \text { on }(0, T) \times \Gamma_{s}\end{cases}
$$

where

$$
F=-w_{t t}-w-\mu w_{t}-\mu_{d} z^{r_{d}}-\int_{-r_{c}}^{0} \mu_{c} z \mathrm{~d} \theta+G_{s} \in L^{2}\left(0, T ; L^{2}\left(\Omega_{s}\right)\right) .
$$

Note that the commutator $\left[\operatorname{div} \sigma, \chi_{1}\right]$ is of order 1 and since $w \in L^{\infty}\left(0, T ; H^{1}\left(\Omega_{s}\right)\right)$, we have $\left[\operatorname{div} \sigma, \chi_{1}\right] w \in L^{\infty}\left(0, T ; L^{2}\left(\Omega_{s}\right)\right)$. Therefore $\chi_{1} w \in L^{2}\left(0, T ; H^{2}\left(\Omega_{s}\right)\right)$ by elliptic regularity, and so for every $\delta>0$ small enough there holds $w \in L^{2}\left(0, T ; H^{2}\left(\Omega_{s}^{\delta}\right)\right)$.

Step 6. Interior regularity of the fluid component. For each $\delta>0$, we define

$$
\Omega_{f}^{\delta}:=\left\{x \in \Omega_{f}: \operatorname{dist}\left(x, \Gamma_{s}\right)>\delta\right\} .
$$

Given $\delta>0$, let $\chi_{2} \in C_{0}^{\infty}\left(\Omega_{f}^{\delta / 4} \cup \Gamma_{f}\right)$ such that $\chi_{2}=1$ on $\Omega_{f}^{\delta / 2} \cup \Gamma_{f}$. Multiplying $\chi_{2}$ to the fluid equation yields

$$
\begin{cases}-\Delta\left(\chi_{2} u\right)+\nabla\left(\chi_{2} p\right)=R_{\chi_{2}}, & \text { in }(0, T) \times \Omega_{f} \\ \operatorname{div}\left(\chi_{2} u\right)=\nabla \chi_{2} \cdot u, & \text { in }(0, T) \times \Omega_{f} \\ \chi_{2} u=0, & \text { in }(0, T) \times \partial \Omega_{f} .\end{cases}
$$

where

$$
R_{\chi_{2}}=\chi_{2} G_{f}-\left(\chi_{2} u_{t}+\left[\Delta, \chi_{2}\right] u+\chi_{2}(u \cdot \nabla) u+\left[\nabla, \chi_{2}\right] p\right) \in L^{2}\left(0, T ; L^{\frac{3}{2}}\left(\Omega_{f}\right)\right)
$$

since the commutators $\left[\Delta, \chi_{2}\right]$ and $\left[\nabla, \chi_{2}\right]$ have orders 1 and 0 , respectively.

Notice that we have the compatibility condition

$$
\int_{\Omega_{f}} \nabla \chi_{2} \cdot u \mathrm{~d} x=\int_{\partial \Omega_{f}} \chi_{2} u \cdot \nu \mathrm{d} x=0 .
$$

According to [43, Proposition 2.2] we have $\chi_{2} u \in L^{2}\left(0, T ; W^{2, \frac{3}{2}}\left(\Omega_{f}\right)\right)$, so that in particular there holds $u \in L^{2}\left(0, T ; W^{2, \frac{3}{2}}\left(\Omega_{f}^{\delta / 2}\right)\right)$, where we also used the fact that $u_{t} \in L^{2}\left(0, T ; L^{\frac{3}{2}}\left(\Omega_{f}\right)\right)$. 
By the Sobolev embedding, $u \in L^{\infty}\left(0, T ; L^{q}\left(\Omega_{f}\right)\right)$ for $2 \leq q<\infty$. In a similar way, since $W^{1, \frac{3}{2}}\left(\Omega_{f}\right) \subset L^{\frac{9}{4}}\left(\Omega_{f}\right)$, we see that $\nabla u \in L^{2}\left(0, T ; L^{\frac{9}{4}}\left(\Omega_{f}^{\delta / 2}\right)\right)$. From Hölder's inequality

$$
\|(u \cdot \nabla) u\|_{L^{2}\left(0, T ; L^{2}\left(\Omega_{f}^{\delta / 2}\right)\right)} \leq C\|u\|_{L^{\infty}\left(0, T ; L^{18}(\Omega)\right)}\|\nabla u\|_{L^{2}\left(0, T ; L^{\frac{9}{4}}\left(\Omega_{f}^{\delta / 2}\right)\right)} .
$$

Thus $(u \cdot \nabla) u \in L^{2}\left(0, T ; L^{2}\left(\Omega_{f}^{\delta / 2}\right)\right)$.

We repeat the above procedure, but now use a cut-off function $\chi_{3} \in C_{0}^{\infty}\left(\Omega_{f}^{\delta / 2} \cup \Gamma_{f}\right)$ such that $\chi_{3}=1$ on $\Omega_{f}^{\delta} \cup \Gamma_{f}$ instead of $\chi_{2}$. Then $R_{\chi_{3}} \in L^{2}\left(0, T ; L^{2}\left(\Omega_{f}^{\delta / 2}\right)\right)$ and hence $\chi_{3} u \in L^{2}\left(0, T ; H^{2}\left(\Omega_{f}\right)\right)$ and $\chi_{3} p \in L^{2}\left(0, T ; H^{1}\left(\Omega_{f}\right)\right)$. Thus, for each small enough $\delta>0$, we have $u \in L^{2}\left(0, T ; H^{2}\left(\Omega_{f}^{\delta}\right)\right)$ and $p \in L^{2}\left(0, T ; H^{1}\left(\Omega_{f}^{\delta}\right)\right)$.

Step 7. Transformation to a problem on a half-space. We adapt the proof in [10]. Consider the neighborhood

$$
\Gamma_{s}^{\delta_{0}}=\left\{x \in \Omega: \operatorname{dist}\left(x, \Gamma_{s}\right)<\delta_{0}\right\}
$$

of the interface where $\delta<\delta_{0}$. Let $\left\{\varrho_{j}\right\}_{j=1}^{N}$ be a partition of unity subordinate to an open cover $\left\{B_{j}\right\}_{j=1}^{N}$ of $\Gamma_{s}^{\delta_{0}}$, that is, $\varrho_{j} \in C_{0}^{\infty}\left(B_{j}\right)$ for each $j$ and $\sum_{j=1}^{N} \varrho_{j}=1$ on $\Gamma_{s}^{\delta_{0}}$. For simplicity, we take $B_{j}$ to be an open disk centered at some point on $\Gamma_{s}$ for each $j$.

Given a function $f$, we denote by $f^{j}$ the product $\varrho_{j} f$. Multiplying the Navier-Stokes and Lamé's equations by the cut-off function $\varrho_{j}$, we obtain the following equations in the sense of distributions

$$
\begin{cases}u_{t}^{j}-\Delta u^{j}+\sum_{i=1}^{N}\left(u^{i} \cdot \nabla\right) u^{j}+\nabla p^{j}=r_{1}^{j} & \text { in }(0, T) \times \Omega_{f}, \\ \operatorname{div} u^{j}=r_{2}^{j}, & \text { in }(0, T) \times \Omega_{f}, \\ u^{j}=0, & \text { on }(0, T) \times \Gamma_{f}, \\ u^{j}=w_{t}^{j}, & \text { on }(0, T) \times \Gamma_{s}, \\ w_{t t}^{j}-\operatorname{div} \sigma\left(w^{j}\right)+w^{j}=G_{s}+r_{3}^{j}, & \text { in }(0, T) \times \Omega_{s}, \\ \sigma\left(w^{j}\right) \cdot \nu=\frac{\partial u^{j}}{\partial \nu}-p^{j} \nu+\frac{1}{2} \sum_{i=1}^{N}\left(u^{i} \cdot \nu\right) u^{j}+r_{4}^{j}, & \text { on }(0, T) \times \Gamma_{s},\end{cases}
$$

where the terms on the right hand sides are given by

$$
\begin{aligned}
& r_{1}^{j}=G_{f}^{j}-\sum_{i=1}^{N}\left[\Delta, \varrho_{j}\right] u^{i}+\sum_{i=1}^{N}\left[\nabla, \varrho_{j}\right] p^{i}+\sum_{i, k=1}^{N}\left(u^{i} \otimes u^{k}\right) \nabla \varrho_{j} \\
& r_{2}^{j}=\sum_{i=1}^{N} \nabla \varrho_{j} \cdot u^{i} \\
& r_{3}^{j}=G_{s}^{j}-\sum_{i=1}^{N}\left[\operatorname{div} \sigma, \varrho_{j}\right] w^{i}-\mu w_{t}^{j}-\mu_{d}\left(z^{j}\right)^{r_{d}}-\int_{-r_{c}}^{0} \mu_{c}(\theta) z^{j}(\theta) \mathrm{d} \theta \\
& r_{4}^{j}=\sum_{i=1}^{N}\left[\sigma \cdot \nu, \varrho_{j}\right] w^{i}-\sum_{i=1}^{N}\left[\partial_{\nu}, \varrho_{j}\right] u^{i},
\end{aligned}
$$

and with initial conditions $u^{j}(0)=u_{0}^{j}, w^{j}(0)=w_{0}^{j}, w_{t}^{j}(0)=w_{0}^{j}$ and initial history $z^{j}(0)=$ $z_{0}^{j}$. Utilizing the Poisson bracket, observe that the commutators $\left[\Delta, \varrho_{j}\right]$ and $\left[\operatorname{div} \sigma, \varrho_{j}\right]$ are differential operators of order 1 , while $\left[\nabla, \varrho_{j}\right]$ is a zero order differential operator. Similarly, the commutators $\left[\sigma \cdot \nu, \varrho_{j}\right]$ and $\left[\partial_{\nu}, \varrho_{j}\right]$ are zero order boundary trace operators on $\Gamma_{s}$. Note 
that in fact, the sum appearing in the terms $r_{l}^{j}$ for $l=1,2,3,4$ can be taken for all indices $i$ such that the supports of $\varrho_{i}$ and $\varrho_{j}$ are nonempty.

We now transform problem (6.13) in such a way that locally the interface $\Gamma_{s}$ is mapped to a flat boundary. Consider the Melrose-Sjöstrand transformations $\Psi_{f}^{j}:\left(\Omega_{f} \cup \Gamma_{s}\right) \cap B_{j} \rightarrow$ $[0,1) \times(-1,1)$ and $\Psi_{s}^{j}:\left(\Omega_{s} \cup \Gamma_{s}\right) \cap B_{j} \rightarrow(-1,0] \times(-1,1)$ such that

$$
\begin{aligned}
& \Omega_{f}^{*}:=\Psi_{f}^{j}\left(\Omega_{f} \cap B_{j}\right)=(0,1) \times(-1,1), \\
& \Omega_{s}^{*}:=\Psi_{f}^{j}\left(\Omega_{s} \cap B_{j}\right)=(-1,0) \times(-1,1), \\
& \Gamma_{s}^{*}:=\Psi_{f}^{j}\left(\Gamma_{s} \cap B_{j}\right)=\Psi_{s}^{j}\left(\Gamma_{s} \cap B_{j}\right)=\{0\} \times(-1,1) .
\end{aligned}
$$

Let $(x, y)$ denote the coordinates of the transformed domains. Under these change of coordinates, the Laplacian $\Delta$ in $\Omega_{f}$ and the operator $\operatorname{div} \sigma$ in $\Omega_{s}$ are transformed into $\Delta_{j f}:=\partial_{x}^{2}+\rho_{f}^{j}(x, y) \partial_{y}^{2}+R_{f}^{j}\left(x, y, \partial_{y}\right)$ and $\Delta_{j s}=\partial_{x}^{2}+\rho_{s}^{j}(x, y) \partial_{y}^{2}+R_{s}^{j}\left(x, y, \partial_{y}\right)$, respectively, for some non-vanishing smooth $\rho_{f}^{j}$ and $\rho_{s}^{j}$, and first order pseudo-differential operators $R_{f}^{j}$ and $R_{s}^{j}$. Likewise, the boundary operators $\partial_{\nu}$ and $\sigma \cdot \nu$ are transformed into $\left.\partial_{x}\right|_{x=0}$. On the other hand, the Dirichlet boundary conditions are invariant under the above transformations.

Let us consider the transformed variables

$$
\tilde{u}^{j}=u^{j} \circ\left(\Psi_{f}^{j}\right)^{-1}, \quad \tilde{p}^{j}=p^{j} \circ\left(\Psi_{f}^{j}\right)^{-1}, \quad \tilde{w}^{j}=w^{j} \circ\left(\Psi_{s}^{j}\right)^{-1}, \quad \tilde{z}^{j}=u^{j} \circ\left(\Psi_{s}^{j}\right)^{-1}
$$

for each $j=1, \ldots, N$. We shall use the same notation for the source terms and initial data. Also, let $\tilde{u}=\left(\tilde{u}^{1}, \ldots, \tilde{u}^{N}\right)$ and similar for the other state variables. By construction, $\tilde{u}^{j}$ and $\tilde{p}^{j}$ vanish on regions in $\mathbb{R}^{2}$ where $|y| \geq 1$ or $x \geq 1$, while $\tilde{w}^{j}$ and $\tilde{z}^{j}$ vanish on regions where $|y| \geq 1$ or $x \leq-1$. Retaining the principal part of the Laplacian on the left hand side, the system (6.13) can now be rewritten in the following form

$$
\begin{cases}\tilde{u}_{t}^{j}-\operatorname{div}\left(A_{f}^{j} \nabla \tilde{u}^{j}\right)+\sum_{i=1}^{N}\left(A_{f}^{j} \tilde{u}^{i} \cdot \nabla\right) \tilde{u}^{j}+A_{f}^{j} \nabla \tilde{p}^{j} & \\ \quad=\tilde{G}_{f}^{j}+\ell_{1}(\tilde{u}, \nabla \tilde{u}, \tilde{p}, \tilde{u} \otimes \tilde{u}), & \text { in }(0, T) \times \Omega_{f}^{*}, \\ \operatorname{div} \tilde{u}^{j}=\ell_{2}(\tilde{u}), & \text { in }(0, T) \times \Omega_{f}^{*}, \\ \tilde{u}^{j}=0, & \text { on }(0, T) \times \Gamma_{f}^{*}, \\ \tilde{u}^{j}=\tilde{w}_{t}^{j}, & \text { on }(0, T) \times \Gamma_{s}^{*}, \\ \tilde{w}_{t t}^{j}-\operatorname{div}\left(A_{s}^{j} \nabla \tilde{w}^{j}\right)+\tilde{w}^{j} & \\ \quad=\tilde{G}_{s}^{j}+\ell_{3}\left(\tilde{w}_{t}, \tilde{w}, \nabla \tilde{w}, \tilde{z}^{r_{d}}, \int_{-r_{c}}^{0} \mu_{c}(\theta) \tilde{z}(\theta) \mathrm{d} \theta\right), & \text { in }(0, T) \times \Omega_{s}^{*}, \\ \frac{\partial \tilde{w}^{j}}{\partial x}=\frac{\partial \tilde{u}^{j}}{\partial x}-\tilde{p}^{j} e_{x}+\sum_{i=1}^{N} \frac{1}{2}\left(\tilde{u}^{i} \cdot e_{x}\right) \tilde{u}^{j}+\ell_{4}(\tilde{u}, \tilde{w}), & \\ & \text { on }(0, T) \times \Gamma_{s}^{*},\end{cases}
$$

where $A_{f}^{j}=\operatorname{diag}\left(1, \rho_{f}^{j}\right), A_{s}^{j}=\operatorname{diag}\left(1, \rho_{s}^{j}\right), e_{x}=(1,0)$ and $\ell_{k}$ is a multilinear form with smooth coefficients for every $k=1,2,3,4$. We refer to the Appendix in [8] for the transformed gradient and divergence operators in the M-S coordinates. Note that a priori we 
have

$$
\begin{aligned}
& \tilde{u} \in L^{\infty}\left(0, T ; H^{1}\left(\Omega_{s}^{*}\right)\right), \\
& \tilde{w} \in W^{1, \infty}\left(0, T ; H^{1}\left(\Omega_{s}^{*}\right)\right) \cap W^{2, \infty}\left(0, T ; L^{2}\left(\Omega_{s}^{*}\right)\right), \\
& \tilde{p} \in L^{2}\left(0, T ; L^{2}\left(\Omega_{f}^{*}\right)\right), \\
& \tilde{z} \in L^{2}\left(0, T ; H^{1}\left((-r, 0) \times \Omega_{s}^{*}\right)\right) .
\end{aligned}
$$

In particular, $\tilde{u} \otimes \tilde{u} \in L^{\infty}\left(0, T ; L^{2}\left(\Omega_{f}^{*}\right)\right)$ by the Sobolev embedding theorem.

Step 8. Tangential regularity of the transformed variables. The next step is to apply the tangential derivative $\partial_{y}$ to (6.14). For this, we obtain the following equations for each $j=1, \ldots, N$ in the distributional sense

$$
\begin{cases}\partial_{y} \tilde{u}_{t}^{j}-\operatorname{div}\left(A_{f}^{j} \nabla \partial_{y} \tilde{u}^{j}\right)+A_{f}^{j} \nabla\left(\partial_{y} \tilde{p}^{j}\right) & \\ \quad=\partial_{y} \tilde{G}_{f}^{j}-\sum_{i=1}^{N}\left\{\left(\partial_{y}\left(A_{f}^{j} \tilde{u}^{i}\right) \cdot \nabla\right) \tilde{u}^{j}+\left(A_{f}^{j} \tilde{u}^{i} \cdot \nabla\right) \partial_{y} \tilde{u}^{j}\right\} & \\ \quad+\left[A_{f}^{j} \nabla, \partial_{y}\right] \tilde{p}^{j}-\left[\operatorname{div}\left(A_{f}^{j} \nabla\right), \partial_{y}\right] \tilde{u}^{j}+\partial_{y} \ell_{1}(\tilde{u}, \nabla \tilde{u}, \tilde{p}, \tilde{u} \otimes \tilde{u}), & \text { in }(0, T) \times \Omega_{f}^{*}, \\ \operatorname{div}\left(A_{f}^{j} \partial_{y} \tilde{u}^{j}\right)=\left[\operatorname{div}, A_{f}^{j} \partial_{y}\right] \tilde{u}^{j}+A_{f}^{j} \partial_{y} \ell_{2}(\tilde{u}), & \text { in }(0, T) \times \Omega_{f}^{*}, \\ \partial_{y} \tilde{u}^{j}=0, & \text { on }(0, T) \times \Gamma_{f}^{*}, \\ \partial_{y} \tilde{u}^{j}=\partial_{y} \tilde{w}_{t}^{j}, & \text { on }(0, T) \times \Gamma_{s}^{*}, \\ \partial_{y} \tilde{w}_{t t}^{j}-\operatorname{div}\left(A_{s}^{j} \nabla \partial_{y} \tilde{w}^{j}\right)=\partial_{y} \tilde{G}_{s}^{j}-\left[\operatorname{div}\left(A_{s}^{j} \nabla\right), \partial_{y}\right] \tilde{w}^{j} & \text { in }(0, T) \times \Omega_{s}^{*}, \\ \quad+\partial_{y} \ell_{3}\left(\tilde{w}_{t}, \tilde{w}, \nabla \tilde{w}^{\prime}, \tilde{z}^{r_{d}}, \int_{-r_{c}}^{0} \mu_{c}(\theta) \tilde{z}(\theta) \mathrm{d} \theta\right), & \\ \frac{\partial}{\partial x}\left(\partial_{y} \tilde{w}^{j}\right)=\frac{\partial}{\partial x}\left(\partial_{y} \tilde{u}^{j}\right)-\partial_{y} \tilde{p}^{j} e_{x} & \\ \quad+\frac{1}{2} \sum_{i=1}^{N}\left\{\left(\partial_{y} \tilde{u}^{i} \cdot e_{x}\right) \tilde{u}^{j}+\left(\tilde{u}^{i} \cdot e_{x}\right) \partial_{y} \tilde{u}^{j}\right\}+\partial_{y} \ell_{4}(\tilde{u}, \tilde{w}), & \text { on }(0, T) \times \Gamma_{s}^{*} .\end{cases}
$$

For each $j=1, \ldots, N$, define the energy of the transformed variables as follows

$$
\tilde{\mathscr{E}}_{j}(t)=\left\{\left\|\partial_{y} \tilde{u}^{j}(t)\right\|_{\Omega_{f}^{*}}^{2}+\left\|\partial_{y} \tilde{w}_{t}^{j}(t)\right\|_{\Omega_{s}^{*}}^{2}+\left\|\partial_{y} \tilde{w}^{j}(t)\right\|_{\Omega_{s}^{*}}^{2}+\left(A_{s}^{j} \nabla \partial_{y} \tilde{w}^{j}(t), \nabla \partial_{y} \tilde{w}^{j}(t)\right)_{\Omega_{s}^{*}}\right.
$$

In the remaining parts of the proof, $C$ will denote a generic positive constant that depends on the norms of $\tilde{u}, \tilde{w}, \tilde{z}, \tilde{p}$ that are known to be finite, and as well on the norms of the initial data, initial history and the source terms. A subscript for $C$ will be used to denote dependence on other parameters. By a standard energy method, we obtain

$$
\tilde{\mathscr{E}}_{j}(t)+\int_{0}^{T}\left\|\partial_{y} \tilde{u}^{j}\right\|_{H^{1}\left(\Omega_{f}^{*}\right)}^{2} \mathrm{~d} s \leq C \tilde{\mathscr{E}}_{j}(0)+C \sum_{k=1}^{4} \int_{0}^{T} \tilde{\mathscr{D}}_{j k}(s) \mathrm{d} s, \quad j=1, \ldots, N
$$


for every $t \in[0, T]$, where $\tilde{\mathscr{D}}_{j k}$ are given as follows

$$
\begin{aligned}
\tilde{\mathscr{D}}_{j 1}= & -\left(\tilde{G}_{f}^{j}+\ell_{1}(\tilde{u}, \nabla \tilde{u}, \tilde{p}, \tilde{u} \otimes \tilde{u}), \partial_{y}^{2} \tilde{u}^{j}\right)_{\Omega_{f}^{*}}+\left(\left[A_{f}^{j} \nabla, \partial_{y}\right] \tilde{p}^{j}, \partial_{y} \tilde{u}^{j}\right)_{\Omega_{f}^{*}} \\
& -\left(\left[\operatorname{div}\left(A_{f}^{j} \nabla\right), \partial_{y}\right] \tilde{u}^{j}, \partial_{y} \tilde{u}^{j}\right)_{\Omega_{f}^{*}}-\sum_{i=1}^{N}\left\{b\left(\partial_{y}\left(A_{f}^{j} \tilde{u}^{i}\right), \tilde{u}^{j}, \partial_{y} \tilde{u}^{j}\right)+b\left(A_{f}^{j} \tilde{u}^{i}, \partial_{y} \tilde{u}^{j}, \partial_{y} \tilde{u}^{j}\right)\right\} \\
\tilde{D}_{j 2}= & \left(\partial_{y} \tilde{p}^{j},\left[\operatorname{div}, A_{f}^{j} \partial_{y}\right] \tilde{u}^{j}+A_{f}^{j} \partial_{y} \ell_{2}(\tilde{u})\right)_{\Omega_{f}^{*}} \\
\tilde{\mathscr{D}}_{j 3}= & \left(\partial_{y} \tilde{G}_{s}^{j}+\partial_{y} \ell_{3}\left(\tilde{w}_{t}, \tilde{w}, \nabla \tilde{w}, \tilde{z}^{r_{d}}, \int_{-r_{c}}^{0} \mu_{c}(\theta) \tilde{z}(\theta) \mathrm{d} \theta\right), \partial_{y} \tilde{w}_{t}^{j}\right)_{\Omega_{s}^{*}} \\
& -\left(\left[\operatorname{div}\left(A_{s}^{j} \nabla\right), \partial_{y}\right] \tilde{w}^{j}, \partial_{y} \tilde{w}_{t}^{j}\right)_{\Omega_{s}^{*}} \\
\tilde{\mathscr{D}}_{j 4}= & \left(\partial_{y} \ell_{4}(\tilde{u}, \tilde{w}), \partial_{y} \tilde{u}^{j}\right)_{\Gamma_{s}^{*}} .
\end{aligned}
$$

Let us estimate the integral over $[0, T]$ of each of the functions $\mathscr{D}_{j k}$. First, note that the commutator $\left[\operatorname{div}\left(A_{f}^{j} \nabla\right), \partial_{y}\right]$ is a second order tangential differential operator. Hence, integrating by parts in the direction of $y$, one obtains

$$
\begin{aligned}
& \int_{0}^{T}\left|\left(\left[\operatorname{div}\left(A_{f}^{j} \nabla\right), \partial_{y}\right] \tilde{u}^{j}, \partial_{y} \tilde{u}^{j}\right)_{\Omega_{f}^{*}}\right| \mathrm{d} s \\
& \quad \leq C_{\varepsilon} \int_{0}^{T}\left\|\tilde{u}^{j}\right\|_{H^{1}\left(\Omega_{f}^{*}\right)}^{2} \mathrm{~d} s+\varepsilon \int_{0}^{T}\left\|\partial_{y} \tilde{u}^{j}\right\|_{H^{1}\left(\Omega_{f}^{*}\right)}^{2} \mathrm{~d} s .
\end{aligned}
$$

On the other hand, the principal part of the commutator $\left[A_{f}^{j} \nabla, \partial_{y}\right]$ is a first order tangential differential operator, hence we have

$$
\begin{aligned}
& \int_{0}^{T}\left|\left(\left[A_{f}^{j} \nabla, \partial_{y}\right] \tilde{p}^{j}, \partial_{y} \tilde{u}^{j}\right)_{\Omega_{f}^{*}}\right| \mathrm{d} s \\
& \quad \leq C_{\varepsilon} \int_{0}^{T}\left\{\left\|\tilde{p}^{j}\right\|_{L^{2}\left(\Omega_{f}^{*}\right)}^{2}+\left\|\partial_{y} \tilde{u}^{j}\right\|_{L^{2}\left(\Omega_{f}^{*}\right.}^{2}\right\} \mathrm{d} s+\varepsilon \int_{0}^{T}\left\|\partial_{y} \tilde{u}^{j}\right\|_{H^{1}\left(\Omega_{f}^{*}\right)}^{2} \mathrm{~d} s .
\end{aligned}
$$

Now let us estimate the trilinear terms in $\tilde{\mathscr{D}}_{j 1}$. Since $\partial_{y}\left(A_{f}^{j} \tilde{u}^{j}\right)$ is no longer divergence free in $\Omega_{f}^{*}$, we cannot apply Lemma 4.3 directly. Nevertheless, by using Hölder's inequality, the Sobolev embedding $H^{\frac{1}{2}}\left(\Omega_{f}^{*}\right) \subset L^{4}\left(\Omega_{f}^{*}\right)$, interpolation and Young's inequality, we obtain

$$
\begin{aligned}
& \left.\int_{0}^{T} \mid\left(\partial_{y}\left(A_{f}^{j} \tilde{u}^{i}\right) \cdot \nabla\right) \tilde{u}^{j}, \partial_{y} \tilde{u}^{j}\right)_{\Omega_{f}^{*}} \mid \mathrm{d} s \\
& \quad \leq \int_{0}^{T}\left\|\partial_{y}\left(A_{f}^{j} \tilde{u}^{i}\right)\right\|_{L^{4}\left(\Omega_{f}^{*}\right)}\left\|\nabla \tilde{u}^{j}\right\|_{L^{2}\left(\Omega_{f}^{*}\right)}\left\|\partial_{y} \tilde{u}^{j}\right\|_{L^{4}\left(\Omega_{f}^{*}\right)} \mathrm{d} s \\
& \quad \leq \int_{0}^{T}\left\|\partial_{y}\left(A_{f}^{j} \tilde{u}^{i}\right)\right\|_{H^{\frac{1}{2}\left(\Omega_{f}^{*}\right)}}\left\|\tilde{u}^{j}\right\|_{H^{1}\left(\Omega_{f}^{*}\right)}\left\|\partial_{y} \tilde{u}^{j}\right\|_{H^{\frac{1}{2}\left(\Omega_{f}^{*}\right)}} \mathrm{d} s \\
& \quad \leq C\left\|\tilde{u}^{j}\right\|_{L^{\infty}\left(0, T ; H^{1}\left(\Omega_{f}^{*}\right)\right)} \int_{0}^{T}\left\{\left\|\partial_{y}\left(A_{f}^{j} \tilde{u}^{i}\right)\right\|_{H^{\frac{1}{2}\left(\Omega_{f}^{*}\right)}}^{2}+\left\|\partial_{y} \tilde{u}^{j}\right\|_{H^{\frac{1}{2}}\left(\Omega_{f}^{*}\right)}^{2}\right\} \mathrm{d} s \\
& \quad \leq C \int_{0}^{T}\left\{\left\|\partial_{y}\left(A_{f}^{j} \tilde{u}^{i}\right)\right\|_{L^{2}\left(\Omega_{f}^{*}\right)}\left\|\partial_{y}\left(A_{f}^{j} \tilde{u}^{i}\right)\right\|_{H^{1}\left(\Omega_{f}^{*}\right)}+\left\|\partial_{y} \tilde{u}^{j}\right\|_{L^{2}\left(\Omega_{f}^{*}\right)}\left\|\partial_{y} \tilde{u}^{j}\right\|_{H^{1}\left(\Omega_{f}^{*}\right)}\right\} \mathrm{d} s \\
& \quad \leq C_{\varepsilon}+\varepsilon \int_{0}^{T}\left\|\partial_{y} \tilde{u}^{i}\right\|_{H^{1}\left(\Omega_{f}^{*}\right)}^{2} \mathrm{~d} s+\varepsilon \int_{0}^{T}\left\|\partial_{y} \tilde{u}^{j}\right\|_{H^{1}\left(\Omega_{f}^{*}\right)}^{2} \mathrm{~d} s
\end{aligned}
$$


Using Lemma 3.4 in [9] along with the same process as above, we have the following estimate

$$
\begin{aligned}
& \int_{0}^{T}\left|\left(\left(\partial_{y} \tilde{u}^{i} \cdot e_{x}\right) \tilde{u}^{j}, \partial_{y} \tilde{u}^{j}\right)_{\Gamma_{s}^{*}}\right| \mathrm{d} t \\
& \quad \leq \int_{0}^{T}\left\|\partial_{y} \tilde{u}^{i}\right\|_{H^{\frac{1}{2}}\left(\Omega_{f}^{*}\right)}\left\|\tilde{u}^{j}\right\|_{H^{\frac{3}{4}}\left(\Omega_{f}^{*}\right)}\left\|\partial_{y} \tilde{u}^{j}\right\|_{H^{\frac{3}{4}\left(\Omega_{f}^{*}\right)}} \mathrm{d} s \\
& \quad \leq C\left\|\tilde{u}^{j}\right\|_{L^{\infty}\left(0, T ; H^{1}\left(\Omega_{f}^{*}\right)\right)} \int_{0}^{T}\left\{\left\|\partial_{y} \tilde{u}^{i}\right\|_{H^{\frac{1}{2}}\left(\Omega_{f}^{*}\right)}^{2}+\left\|\partial_{y} \tilde{u}^{j}\right\|_{H^{\frac{3}{4}}\left(\Omega_{f}^{*}\right)}^{2}\right\} \mathrm{d} s \\
& \quad \leq C \int_{0}^{T}\left\{\left\|\partial_{y} \tilde{u}^{i}\right\|_{L^{2}\left(\Omega_{f}^{*}\right.}\left\|\partial_{y} \tilde{u}^{i}\right\|_{H^{1}\left(\Omega_{f}^{*}\right)}+\left\|\partial_{y} \tilde{u}^{j}\right\|_{L^{2}\left(\Omega_{f}^{*}\right)}^{1 / 2}\left\|\partial_{y} \tilde{u}^{j}\right\|_{H^{1}\left(\Omega_{f}^{*}\right)}^{3 / 2}\right\} \mathrm{d} s \\
& \quad \leq C_{\varepsilon}+\varepsilon \int_{0}^{T}\left\|\partial_{y} \tilde{u}^{i}\right\|_{H^{1}\left(\Omega_{f}^{*}\right)}^{2} \mathrm{~d} s+\varepsilon \int_{0}^{T}\left\|\partial_{y} \tilde{u}^{j}\right\|_{H^{1}\left(\Omega_{f}^{*}\right)}^{2} \mathrm{~d} s .
\end{aligned}
$$

Thus, from (6.17) and (6.18), the first trilinear term in $\tilde{\mathscr{D}}_{j 1}$ can be estimated as follows

$$
\begin{aligned}
& \sum_{i=1}^{N} \int_{0}^{T}\left|b\left(\partial_{y}\left(A_{f}^{j} \tilde{u}^{i}\right), \tilde{u}^{j}, \partial_{y} \tilde{u}^{j}\right)\right| \mathrm{d} s \\
& \quad \leq C_{\varepsilon}+2 N \varepsilon \int_{0}^{T}\left\|\partial_{y} \tilde{u}\right\|_{H^{1}\left(\Omega_{f}^{*}\right)}^{2} \mathrm{~d} s+2 N \varepsilon \int_{0}^{T}\left\|\partial_{y} \tilde{u}^{j}\right\|_{H^{1}\left(\Omega_{f}^{*}\right)}^{2} \mathrm{~d} s
\end{aligned}
$$

For the second trilinear term in $\tilde{\mathscr{D}}_{j 1}$, we shall invoke the identity

$$
b\left(A_{f}^{j} \tilde{u}^{i}, \partial_{y} \tilde{u}^{j}, \partial_{y} \tilde{u}^{j}\right)=\frac{1}{2}\left(\operatorname{div}\left(A_{f}^{j} \tilde{u}^{i}\right), \partial_{y} \tilde{u}^{j} \otimes \partial_{y} \tilde{u}^{j}\right)_{\Omega_{f}^{*}},
$$

apply Hölder's inequality and use Sobolev embedding

$$
\begin{aligned}
& \sum_{i=1}^{N} \int_{0}^{T}\left|b\left(A_{f}^{j} \tilde{u}^{i}, \partial_{y} \tilde{u}^{j}, \partial_{y} \tilde{u}^{j}\right)\right| \mathrm{d} s \\
& \quad \leq C \sum_{i=1}^{N} \int_{0}^{T}\left\|\operatorname{div}\left(A_{f}^{j} \tilde{u}^{i}\right)\right\|_{L^{2}\left(\Omega_{f}^{*}\right)}\left\|\partial_{y} \tilde{u}^{j}\right\|_{L^{4}\left(\Omega_{f}^{*}\right)}^{2} \mathrm{~d} s \\
& \quad \leq C \sum_{i=1}^{N} \int_{0}^{T}\left\|\tilde{u}^{i}\right\|_{H^{1}\left(\Omega_{f}^{*}\right)}\left\|\partial_{y} \tilde{u}^{j}\right\|_{H^{\frac{1}{2}}\left(\Omega_{f}^{*}\right)}^{2} \mathrm{~d} s \\
& \quad \leq C \sum_{i=1}^{N} \int_{0}^{T}\left\|\tilde{u}^{i}\right\|_{H^{1}\left(\Omega_{f}^{*}\right)}\left\|\partial_{y} \tilde{u}^{j}\right\|_{L^{2}\left(\Omega_{f}^{*}\right)}\left\|\partial_{y} \tilde{u}^{j}\right\|_{H^{1}\left(\Omega_{f}^{*}\right)} \mathrm{d} s \\
& \quad \leq C_{\varepsilon}+N \varepsilon \int_{0}^{T}\left\|\partial_{y} \tilde{u}^{j}\right\|_{H^{1}\left(\Omega_{f}^{*}\right)}^{2} \mathrm{~d} s .
\end{aligned}
$$

For the remaining term in $\tilde{\mathscr{D}}_{j 1}$, observe that $\tilde{G}_{f}^{j}, \ell_{1}(\tilde{u}, \nabla \tilde{u}, \tilde{p}, \tilde{u} \otimes \tilde{u}) \in L^{2}\left(0, T ; L^{2}\left(\Omega_{f}^{*}\right)\right)$, and therefore we have

$$
\int_{0}^{T}\left|\left(\ell_{1}(\tilde{u}, \nabla \tilde{u}, \tilde{p}, \tilde{u} \otimes \tilde{u})+\tilde{G}_{f}^{j}, \partial_{y}^{2} \tilde{u}^{j}\right)_{\Omega_{f}^{*}}\right| \mathrm{d} s \leq C_{\varepsilon}+\varepsilon \int_{0}^{T}\left\|\partial_{y} \tilde{u}^{j}\right\|_{H^{1}\left(\Omega_{f}^{*}\right)}^{2} \mathrm{~d} s .
$$

Combining the estimates (6.15), (6.16), (6.19)-(6.21), we obtain

$$
\int_{0}^{T} \tilde{\mathscr{D}}_{j 1}(s) \mathrm{d} s \leq C_{\varepsilon}+C_{N} \varepsilon \int_{0}^{T}\left\|\partial_{y} \tilde{u}\right\|_{H^{1}\left(\Omega_{f}^{*}\right)}^{2} \mathrm{~d} s+C_{N} \varepsilon \int_{0}^{T}\left\|\partial_{y} \tilde{u}^{j}\right\|_{H^{1}\left(\Omega_{f}^{*}\right)}^{2} \mathrm{~d} s .
$$


Notice that the principal part of the commutator $\left[\operatorname{div}, A_{f}^{j} \partial_{y}\right]$ is a first order differential operator in the tangential direction. Thus, integrating by parts in the direction of $y$ yields the following estimate for $\tilde{\mathscr{D}}_{2}$

$$
\int_{0}^{T} \tilde{\mathscr{D}}_{j 2}(s) \mathrm{d} s \leq C_{\varepsilon} \int_{0}^{T}\left\{\left\|\tilde{p}^{j}\right\|_{L^{2}\left(\Omega_{f}^{*}\right)}^{2}+\left\|\partial_{y} \tilde{u}^{j}\right\|_{L^{2}\left(\Omega_{f}^{*}\right)}^{2}\right\} \mathrm{d} s+\varepsilon \int_{0}^{T}\left\|\partial_{y} \tilde{u}^{j}\right\|_{H^{1}\left(\Omega_{f}^{*}\right)}^{2} \mathrm{~d} s .
$$

Since $\tilde{w} \in W^{1, \infty}\left(0, T ; H^{1}\left(\Omega_{s}^{*}\right)\right)$ and $\partial_{y} \tilde{G}_{s}^{j}, \tilde{z}^{r_{d}}, \int_{-r_{c}}^{0} \mu_{s}(\theta) \tilde{z}(\theta) \mathrm{d} \theta \in L^{2}\left(0, T ; L^{2}\left(\Omega_{s}^{*}\right)\right)$, one can bound the term $\tilde{\mathscr{D}}_{j 3}$ as

$$
\begin{aligned}
& \int_{0}^{T} \tilde{\mathscr{D}}_{j 3}(t) \mathrm{d} s \leq C+C \int_{0}^{T}\left\|\partial_{y} \tilde{w}\right\|_{H^{1}\left(\Omega_{s}^{*}\right)}\left\|\partial_{y} \tilde{w}_{t}^{j}\right\|_{L^{2}\left(\Omega_{s}^{*}\right)} \mathrm{d} s \\
& \quad \leq C_{\varepsilon}+\varepsilon\left\|\partial_{y} \tilde{w}\right\|_{L^{\infty}\left(0, T ; H^{1}\left(\Omega_{s}^{*}\right)\right)}^{2}+C_{\varepsilon, T}\|\tilde{w}\|_{W^{1, \infty}\left(0, T ; H^{1}\left(\Omega_{s}^{*}\right)\right)}^{2} .
\end{aligned}
$$

Finally by trace theorem, we can estimate the term $\tilde{\mathscr{D}}_{j 4}$ as follows

$$
\begin{aligned}
& \int_{0}^{T} \tilde{\mathscr{D}}_{j 4}(s) \mathrm{d} s \leq \int_{0}^{T}\left\|\partial_{y} \ell_{4}(\tilde{u}, \tilde{w})\right\|_{H^{-\frac{1}{2}\left(\Gamma_{s}^{*}\right)}}\left\|\partial_{y} \tilde{u}^{j}\right\|_{H^{\frac{1}{2}}\left(\Gamma_{s}^{*}\right)} \mathrm{d} s \\
& \quad \leq C \int_{0}^{T}\left\{\|\tilde{u}\|_{H^{\frac{1}{2}}\left(\Gamma_{s}^{*}\right)}+\|\tilde{w}\|_{H^{\frac{1}{2}\left(\Gamma_{s}^{*}\right)}}\right\}\left\|\partial_{y} \tilde{u}^{j}\right\|_{H^{\frac{1}{2}}\left(\Gamma_{s}^{*}\right)} \mathrm{d} s \\
& \quad \leq C_{\varepsilon} \int_{0}^{T}\left\{\|\tilde{u}\|_{H^{1}\left(\Omega_{s}^{*}\right)}^{2}+\|\tilde{w}\|_{H^{1}\left(\Omega_{s}^{*}\right)}^{2}\right\} \mathrm{d} s+\varepsilon \int_{0}^{T}\left\|\partial_{y} \tilde{u}^{j}\right\|_{H^{1}\left(\Omega_{f}^{*}\right)}^{2} \mathrm{~d} s .
\end{aligned}
$$

In the second inequality, we used Plancherel's identity to obtain

$$
\begin{gathered}
\left\|\partial_{y} \tilde{u}\right\|_{H^{-\frac{1}{2}}\left(\Gamma_{s}^{*}\right)}^{2}=\int_{\mathbb{R}}\left(1+|\eta|^{2}\right)^{-\frac{1}{2}}|\eta|^{2}|(\mathscr{F} \tilde{u})(\eta)|^{2} \mathrm{~d} \eta \\
\quad \leq \int_{\mathbb{R}}\left(1+|\eta|^{2}\right)^{\frac{1}{2}}|(\mathscr{F} \tilde{u})(\eta)|^{2} \mathrm{~d} \eta=\|\tilde{u}\|_{H^{\frac{1}{2}}\left(\Gamma_{s}^{*}\right)}^{2}
\end{gathered}
$$

where $\tilde{u}$ is extended by zero for $|y| \geq 1$ and $\mathscr{F} \tilde{u}$ denotes the Fourier transform of $\tilde{u}$. A similar estimate involving $\tilde{w}$ can be derived.

Using the above bounds for $\tilde{\mathscr{D}}_{j k}$ and invoking the known a priori regularities of the transformed variables, we have

$$
\begin{aligned}
\tilde{\mathscr{E}}_{j}(t) & +\left(1-C_{N} \varepsilon\right) \int_{0}^{T}\left\|\partial_{y} \tilde{u}^{j}\right\|_{H^{1}\left(\Omega_{f}^{*}\right)}^{2} \mathrm{~d} s \\
& \leq C_{\varepsilon, T}+C_{N} \varepsilon \int_{0}^{T}\left\|\partial_{y} \tilde{u}\right\|_{H^{1}\left(\Omega_{f}^{*}\right)}^{2} \mathrm{~d} s+\varepsilon\left\|\partial_{y} \tilde{w}\right\|_{L^{\infty}\left(0, T ; H^{1}\left(\Omega_{s}^{*}\right)\right)}^{2}
\end{aligned}
$$

for every $t \in[0, T]$ and $j=1, \ldots, N$. Taking the sum over all indices $j=1, \ldots, N$ and making $\varepsilon$ sufficiently small, we obtain that $\partial_{y} \tilde{u} \in L^{\infty}\left(0, T ; L^{2}\left(\Omega_{f}^{*}\right)\right) \cap L^{2}\left(0, T ; H^{1}\left(\Omega_{f}^{*}\right)\right)$ and $\partial_{y} \tilde{w} \in L^{\infty}\left(0, T ; H^{1}\left(\Omega_{s}^{*}\right)\right)$.

Step 9. Regularity of nonlinear advection term. In the transformed coordinates we have $\tilde{u} \in L^{2}\left(0, T ; L_{x}^{2}\left(\mathbb{R} ; H_{y}^{2}(\mathbb{R})\right) \cap H_{x}^{1}\left(\mathbb{R} ; H_{y}^{1}(\mathbb{R})\right)\right)$, so that by the Sobolev embedding theorem and the fact that $\tilde{u}$ has compact support we have $\tilde{u} \in L^{2}\left(0, T ; L^{\infty}\left(\mathbb{R}^{2}\right)\right)$. In the original coordinates, this translates into $u \in L^{2}\left(0, T ; L^{\infty}\left(\Omega_{f}\right)\right)$, and because $\nabla u \in C\left(0, T ; L^{2}\left(\Omega_{f}\right)\right)$, it follows that $(u \cdot \nabla) u \in L^{2}\left(0, T ; L^{2}\left(\Omega_{f}\right)\right)$ by Hölder's inequality.

Step 10. $H^{2}$-regularity of the fluid velocity and $H^{1}$-regularity of the pressure. We first establish the regularity in terms of the transformed variables. Indeed, by using $\operatorname{div} \tilde{u}^{j}=\ell_{2}(\tilde{u})$ we have $\partial_{x}^{2} \tilde{u}^{j}=\partial_{x} \ell_{2}(\tilde{u})-\partial_{x y}^{2} \tilde{u}^{j}$ and thus $\partial_{x}^{2} \tilde{u}^{j} \in L^{2}\left(0, T ; L^{2}\left(\Omega_{f}^{*}\right)\right)$ for each $j$. Summing over all estimates obtained on each of the patches yields $\tilde{u} \in L^{2}\left(0, T ; H^{2}\left(\Omega_{f}^{*}\right)\right)$. Finally, from 
the Navier-Stokes equation and the regularity of the nonlinear advection term given in the previous step, we have $\tilde{p} \in L^{2}\left(0, T ; H^{1}\left(\Omega_{f}^{*}\right)\right)$. Translating these to the original coordinates and combining this to the interior regularity from Step 6, we obtain $u \in L^{2}\left(0, T ; H^{2}\left(\Omega_{f}\right)\right)$ and $p \in L^{2}\left(0, T ; H^{1}\left(\Omega_{f}\right)\right)$.

Step 11. $H^{2}$-regularity of solid component. Observe that $\left.\partial_{y} \tilde{w}^{j}\right|_{\Gamma_{s}} \in L^{2}\left(0, T ; H^{\frac{1}{2}}\left(\Gamma_{s}^{*}\right)\right)$, and hence $\left.\tilde{w}^{j}\right|_{\Gamma_{s}^{*}} \in L^{2}\left(0, T ; H^{\frac{3}{2}}\left(\Gamma_{s}^{*}\right)\right)$. Applying elliptic regularity theory to the boundary value problem

$$
\left\{\begin{array}{l}
-\operatorname{div}\left(A_{s}^{j} \nabla \tilde{w}^{j}\right)+\tilde{w}^{j}=-\tilde{w}_{t t}^{j}+\tilde{G}_{s}^{j}+\ell_{3}\left(\tilde{w}_{t}, \tilde{w}, \nabla \tilde{w}, \tilde{z}^{r_{d}}, \int_{-r_{c}}^{0} \mu_{c}(\theta) \tilde{z}(\theta) \mathrm{d} \theta\right), \\
\left.\tilde{w}^{j}\right|_{\Gamma_{s}^{*}} \in L^{2}\left(0, T ; H^{\frac{3}{2}}\left(\Gamma_{s}^{*}\right)\right),
\end{array}\right.
$$

where the right hand side belongs to $L^{2}\left(0, T ; L^{2}\left(\Omega_{s}^{*}\right)\right)$, yields $\tilde{w}^{j} \in L^{2}\left(0, T ; H^{2}\left(\Omega_{s}^{*}\right)\right)$ for each $j=1, \ldots, N$. Combining this with the interior regularity in Step 5, we conclude that $w \in L^{2}\left(0, T ; H^{2}\left(\Omega_{s}\right)\right)$. The proof of the theorem is now complete.

\section{Exponential Stability in Two Dimensions}

In this section, we prove exponential decay of the energy to solutions of (1.1) with $G_{f}=0$ and $G_{s}=0$ under the condition $\mu>\left|\mu_{d}\right|+\left\|\mu_{c}\right\|_{L^{1}\left(-r_{c}, 0\right)}$. This means that the frictional damping should dominate the strengths of the delays. Let

$$
V_{s}=\left\{g \in H^{1}\left(\Omega_{s}\right):(g, \nu)_{\Gamma_{s}}=0\right\}
$$

and $S: V_{s} \rightarrow H^{1}\left(\Omega_{f}\right)$ be the Stokes map defined by $S g=u$ if and only if $u$ is the weak solution of

$$
\begin{cases}-\Delta u+\nabla p=0, & \text { in } \Omega_{f}, \\ \operatorname{div} u=0, & \text { in } \Omega_{f}, \\ u=0, & \text { on } \Gamma_{f}, \\ u=g, & \text { on } \Gamma_{s} .\end{cases}
$$

The map $S$ is linear and bounded thanks to the compatibility condition $(g, \nu)_{\Gamma_{s}}=0$, see for instance [43, Theorem 2.4].

Equilibrium pressure for the fluid is uniquely determined up to an additive constant. Different pressure corresponds to different equilibrium displacement of the elastic body and as a result (1.1) has a one-dimensional manifold of steady states. This has been already observed in the linearized case without delay in [4], see also [38] in the absence of transversal forces. The space of steady states of the fluid-structure model without the source terms is $\{0\} \times \operatorname{span}\left\{w^{*}\right\} \times\{0\} \times\{0\}$, where $w^{*}$ is the weak solution of the elliptic boundary value problem

$$
\left\{\begin{aligned}
-\operatorname{div} \sigma\left(w^{*}\right)+w^{*}=0, & \text { in } \Omega_{s} \\
\sigma\left(w^{*}\right) \cdot \nu=\nu, & \text { on } \Gamma_{s}
\end{aligned}\right.
$$

that is,

$$
a_{s}\left(w^{*}, \psi\right)=\left(\sigma\left(w^{*}\right), \epsilon(\psi)\right)_{\Omega_{s}}+\left(w^{*}, \psi\right)_{\Omega_{s}}=\left(w^{*}, \nu\right)_{\Gamma_{s}}, \text { for all } \psi \in H^{1}\left(\Omega_{s}\right) .
$$

We equip $H^{1}\left(\Omega_{s}\right)$ with the inner product $a_{s}(\cdot, \cdot)$, which is equivalent to the usual $H^{1}\left(\Omega_{s}\right)$ norm according to Korn's inequality. We have the following orthogonal decomposition 
$H^{1}\left(\Omega_{s}\right)=V_{s} \oplus \operatorname{span}\left\{w^{*}\right\}$. Let $\Pi_{V_{s}}$ be the orthogonal projection of $H^{1}\left(\Omega_{s}\right)$ onto $V_{s}$. Decompose the initial displacement of the structure according to

$$
w_{0}=\Pi_{V_{s}} w_{0}+\kappa\left(w_{0}\right) w^{*}, \quad \kappa\left(w_{0}\right)=\frac{\left(w_{0}, \nu\right)_{\Gamma_{s}}}{\left(w^{*}, \nu\right)_{\Gamma_{s}}} .
$$

By uniqueness, the weak solution of (1.1)-(1.2) can be likewise decomposed into

$$
\left(u, w, w_{t}, v\right)=\left(u, \Pi_{V_{s}} w, w_{t}, z\right)+\left(0, \kappa\left(w_{0}\right) w^{*}, 0,0\right) .
$$

In the following, we shall construct a Lyapunov functional equivalent to the energy of the system. We refer to [37] for a related Lyapunov functional for wave equations with internal distributed delay. Before we proceed, let us mention related results in the absence of the retarded terms. The exponential stability of solutions with boundary damping on the wave equation has been shown in [27], with interior damping on the wave equation in [30] and a combination of boundary and interior damping in [31].

Theorem 7.1. If $\mu>\left|\mu_{d}\right|+\left\|\mu_{c}\right\|_{L^{1}\left(-r_{c}, 0\right)}$ then the weak solution of (1.1)-(1.2) with $G_{f}=0$ and $G_{s}=0$ is exponentially stable, that is, there exist $M(\mathscr{E}(0)) \geq 1$ and $\varrho(\mathscr{E}(0))>0$ such that for every $t \geq 0$

$$
\|u(t)\|_{\Omega_{f}}+\left\|w(t)-\kappa\left(w_{0}\right) w^{*}\right\|_{\Omega_{s}}+\left\|w_{t}(t)\right\|_{\Omega_{s}}+\|z(t)\|_{L^{2}\left((-r, 0) \times \Omega_{s}\right)} \leq M(\mathscr{E}(0)) e^{-\varrho(\mathscr{E}(0)) t},
$$

where $\mathscr{E}(0)=\mathscr{E}\left(u_{0}, v_{0}, w_{0}, z_{0}\right)$.

Proof. According to the above discussion, it is enough to consider initial data in $H_{f} \times V_{s} \times$ $L^{2}\left(\Omega_{s}\right) \times L^{2}\left((-r, 0) \times \Omega_{s}\right)$, so that $w(t) \in V_{s}$ for each $t \geq 0$. Define $\omega=S\left(\left.w\right|_{\Gamma_{s}}\right)$ and

$$
\mu_{0}(\theta)=\int_{-r_{c}}^{\theta}\left|\mu_{c}(s)\right| \mathrm{d} s, \quad \theta \in\left[-r_{c}, 0\right] .
$$

Note that $\mu_{0} \geq 0, \mu_{0}^{\prime}=\left|\mu_{c}\right|$ and $\left\|\mu_{0}\right\|_{L^{\infty}(-r, 0)} \leq\left\|\mu_{c}\right\|_{L^{1}(-r, 0)}$. We consider the Lyapunov functional

$$
\mathscr{L}(t)=\mathscr{L}_{u, w}(t)+\mathscr{L}_{z}(t)+\alpha \int_{\Omega_{s}} w_{t}(t) \cdot w(t) \mathrm{d} x+\alpha \int_{\Omega_{f}} u(t) \cdot \omega(t) \mathrm{d} x,
$$

where $\mathscr{L}_{u, w}(t)$ and $\mathscr{L}_{z}(t)$ are the functionals given respectively by

$$
\begin{aligned}
& \mathscr{L}_{u, w}(t)=\frac{1}{2} \int_{\Omega_{f}}|u(t)|^{2} \mathrm{~d} x+\frac{1}{2} \int_{\Omega_{s}} \sigma(w(t)): \epsilon(w(t))+|w(t)|^{2}+\left|w_{t}(t)\right|^{2} \mathrm{~d} x \\
& \mathscr{L}_{z}(t)=\frac{\varepsilon}{2} \int_{-r}^{0} \int_{\Omega_{s}}(\beta+\eta \theta)|z(t, \theta)|^{2} \mathrm{~d} x \mathrm{~d} \theta+\frac{1}{2} \int_{-r_{d}}^{0} \int_{\Omega_{s}}(\beta+\eta \theta)|z(t, \theta)|^{2} \mathrm{~d} x \mathrm{~d} \theta \\
& +\frac{1}{2} \int_{-r_{c}}^{0} \int_{\Omega_{s}} \mu_{0}(\theta)|z(t, \theta)|^{2} \mathrm{~d} x \mathrm{~d} \theta
\end{aligned}
$$

and the positive constants $\alpha, \beta, \varepsilon$, and $\eta \in(0, \beta / r)$ are to be specified later. Observe that for sufficiently small $\alpha, \beta, \varepsilon$ and $\eta$, the Lyapunov functional $\mathscr{L}$ and the energy functional $\mathscr{E}$ are equivalent, that is, there exists a constant $C=C(\alpha, \beta, \varepsilon, \eta)>0$ such that

$$
C \mathscr{E}(t) \leq \mathscr{L}(t) \leq(1 / C) \mathscr{E}(t), \text { for every } t \geq 0 .
$$

Here, we work with the approximate solutions from the proof of the existence theorem and pass to the limit later. For simplicity, we remove the indices. Alternatively, one may start with smooth and compatible initial data satisfying condition (A) to have smooth 
solution and apply the density of such initial data in $X$. First, by using the Cauchy-Schwarz inequality, we have the estimate

$$
\begin{aligned}
\frac{d}{d t} \mathscr{L}_{u, w}(t) \leq & -\int_{\Omega_{f}}|\nabla u(t)|^{2} \mathrm{~d} x-\left(\mu-\frac{\left|\mu_{d}\right|}{2}-\frac{\left\|\mu_{c}\right\|_{L^{1}}}{2}\right) \int_{\Omega_{s}}\left|w_{t}(t)\right|^{2} \mathrm{~d} x \\
& +\frac{\left|\mu_{d}\right|}{2} \int_{\Omega_{s}}\left|z^{r_{d}}(t)\right|^{2} \mathrm{~d} x+\frac{1}{2} \int_{-r_{c}}^{0} \int_{\Omega_{s}}\left|\mu_{c}(\theta)\right||z(t, \theta)|^{2} \mathrm{~d} x \mathrm{~d} \theta .
\end{aligned}
$$

Moreover, for each $\xi \in[0, r]$ we have

$$
\begin{aligned}
& \frac{1}{2} \frac{d}{d t} \int_{-\xi}^{0} \int_{\Omega_{s}}(\beta+\eta \theta)|z(t, \theta)|^{2} \mathrm{~d} x \mathrm{~d} \theta \\
& =\frac{\beta}{2} \int_{\Omega_{s}}\left|w_{t}(t)\right|^{2} \mathrm{~d} x-\frac{1}{2}(\beta-\eta \xi) \int_{\Omega_{s}}\left|z^{\xi}(t)\right|^{2} \mathrm{~d} x-\frac{\eta}{2} \int_{-\xi}^{0} \int_{\Omega_{s}}|z(t, \theta)|^{2} \mathrm{~d} \theta \mathrm{d} x .
\end{aligned}
$$

Furthermore, it holds that

$$
\begin{aligned}
& \frac{1}{2} \frac{d}{d t} \int_{-r_{c}}^{0} \int_{\Omega_{s}} \mu_{0}(\theta)|z(t, \theta)|^{2} \mathrm{~d} x \mathrm{~d} \theta \\
& \leq \frac{\left\|\mu_{c}\right\|_{L^{1}}}{2} \int_{\Omega_{s}}\left|w_{t}(t)\right|^{2} \mathrm{~d} x-\frac{1}{2} \int_{-r_{c}}^{0} \int_{\Omega_{s}}\left|\mu_{c}(\theta)\right||z(t, \theta)|^{2} \mathrm{~d} x \mathrm{~d} \theta .
\end{aligned}
$$

Choosing $\xi=r_{d}$ and $\xi=r$ in (7.3) and taking the sum with (7.2) and (7.4) yields

$$
\begin{aligned}
& \frac{d}{d t}\left[\mathscr{L}_{u, w}(t)+\mathscr{L}_{z}(t)\right] \leq-\int_{\Omega_{f}}|\nabla u(t)|^{2} \mathrm{~d} x-K_{1} \int_{\Omega_{s}}\left|w_{t}(t)\right|^{2} \mathrm{~d} x \\
& -K_{2} \int_{\Omega_{s}}\left|z^{r_{d}}(t)\right|^{2} \mathrm{~d} x-K_{3} \int_{\Omega_{s}}\left|z^{r}(t)\right|^{2} \mathrm{~d} x-\eta \int_{-r}^{0} \int_{\Omega_{s}}|z(t, \theta)|^{2} \mathrm{~d} \theta \mathrm{d} x,
\end{aligned}
$$

where

$$
K_{1}=\mu-\frac{\left|\mu_{d}\right|}{2}-\frac{\beta}{2}-\frac{\varepsilon \beta}{2}-\left\|\mu_{c}\right\|_{L^{1}}, \quad K_{2}=\frac{1}{2}\left(\beta-\left|\mu_{d}\right|-\eta r_{d}\right), \quad K_{3}=\frac{\varepsilon}{2}(\beta-\eta r) .
$$

Take $\beta=\left|\mu_{d}\right|+\varepsilon$ with a suitable $\varepsilon>0$. Since $\mu>\left|\mu_{d}\right|+\left\|\mu_{c}\right\|_{L_{1}}$ we can choose $\varepsilon$ small enough and then $\eta$ small enough so that $K_{1}>0, K_{2}>0$ and $K_{3}>0$. In particular, by integrating (7.5) with respect to time and using (7.1), it holds that

$$
\sup _{0 \leq t<\infty}\left(\sigma(w(t)): \epsilon(w(t))+|w(t)|^{2}\right) \leq C \mathscr{E}(0)
$$

With regards to the last two terms in the Lyapunov functional $\mathscr{L}$, we have

$$
\begin{aligned}
& \frac{d}{d t}\left[\int_{\Omega_{s}} w_{t}(t) \cdot w(t) \mathrm{d} x+\int_{\Omega_{f}} u(t) \cdot \omega(t) \mathrm{d} x\right] \\
& =\int_{\Omega_{s}} w_{t t}(t) \cdot w(t)+\left|w_{t}(t)\right|^{2} \mathrm{~d} x+\int_{\Omega_{f}} u_{t}(t) \cdot \omega(t)+\left.u(t) \cdot S w_{t}(t)\right|_{\Gamma_{s}} \mathrm{~d} x \\
& \leq-\int_{\Omega_{f}}\left\{\nabla u(t): \nabla(\omega(t))-\left.u(t) \cdot S w_{t}(t)\right|_{\Gamma_{s}}\right\} \mathrm{d} x-b(u(t), u(t), \omega(t)) \\
& -\int_{\Omega_{s}}\left\{\sigma(w(t)): \epsilon(w(t))+|w(t)|^{2}+\left(\mu w_{t}(t)+\mu_{d} z^{r_{d}}(t)\right) \cdot w(t)-\left|w_{t}(t)\right|^{2}\right\} \mathrm{d} x \\
& -\int_{-r_{c}}^{0} \int_{\Omega_{s}} \mu_{c}(\theta) z(t, \theta) \cdot w(t) \mathrm{d} x \mathrm{~d} \theta .
\end{aligned}
$$


Let us estimate each terms on the right hand side. Using the fact that $\left.u\right|_{\Gamma_{s}}=\left.w_{t}\right|_{\Gamma_{s}}$, applying the Poincare inequality and the boundedness of the Stokes map $S$, we obtain

$$
\left.\left|\int_{\Omega_{f}} u(t) \cdot S w_{t}(t)\right|_{\Gamma_{s}} \mathrm{~d} x\left|\leq C \int_{\Omega_{f}}\right| \nabla u(t)\right|^{2} \mathrm{~d} x .
$$

Using similar procedure along with Young's inequality, we have

$$
\left|\int_{\Omega_{f}} u(t) \cdot \omega(t) \mathrm{d} x\right| \leq \frac{1}{4} \int_{\Omega_{s}}\left(\sigma(w(t)): \epsilon(w(t))+|w(t)|^{2}\right) \mathrm{d} x+C \int_{\Omega_{f}}|\nabla u(t)|^{2} \mathrm{~d} x .
$$

Likewise, it holds that

$$
\begin{aligned}
& \left|\int_{\Omega_{s}}\left(\mu w_{t}(t)+\mu_{d} z^{r_{d}}(t)\right) \cdot w(t) \mathrm{d} x+\int_{-r_{c}}^{0} \int_{\Omega_{s}} \mu_{c}(\theta) z(t, \theta) \cdot w(t) \mathrm{d} x \mathrm{~d} \theta\right| \\
& \leq \frac{1}{4} \int_{\Omega_{s}}|w(t)|^{2} \mathrm{~d} x+C \int_{\Omega_{s}}\left|w_{t}(t)\right|^{2}+\left|z^{r_{d}}(t)\right|^{2} \mathrm{~d} x+C \int_{-r_{c}}^{0} \int_{\Omega_{s}}|z(t, \theta)|^{2} \mathrm{~d} x \mathrm{~d} \theta .
\end{aligned}
$$

For the trilinear term in (7.7), we apply Lemma 4.3(i) and Corollary 4.4 with $\varepsilon=1$ to obtain

$$
|b(u(t), u(t), \omega(t))| \leq\|u(t)\|_{V_{f}}^{2}+C\|u(t)\|_{\Omega_{f}}^{2}\|\omega(t)\|_{V_{f}}^{2}\left(1+\|\omega(t)\|_{\Omega_{f}}^{2 / 3}\right) .
$$

By trace theory and continuity of $S$ we have $\|\omega(t)\|_{V_{f}}^{2} \leq C\left(\sigma(w(t)): \epsilon(w(t))+|w(t)|^{2}\right)$, and consequently from (7.6) we have

$$
|b(u(t), u(t), \omega(t))| \leq C(\mathscr{E}(0)) \int_{\Omega_{f}}|\nabla u(t)|^{2} \mathrm{~d} x .
$$

Using (7.8)-(7.11) in (7.7) and combining the result in (7.5), we obtain that

$$
\begin{aligned}
& \frac{d}{d t} \mathscr{L}(t) \leq-(1-\alpha C(\mathscr{E}(0))) \int_{\Omega_{f}}|\nabla u(t)|^{2} \mathrm{~d} x-\left(K_{1}-\alpha C\right) \int_{\Omega_{s}}\left|w_{t}(t)\right|^{2} \mathrm{~d} x \\
& -\frac{1}{2} \int_{\Omega_{s}} \sigma(w(t)): \epsilon(w(t))+|w(t)|^{2} \mathrm{~d} x-\left(K_{2}-\alpha C\right) \int_{\Omega_{s}}\left|z^{r_{d}}(t)\right|^{2} \mathrm{~d} x \\
& -\left(\frac{\eta}{2}-\alpha C\right) \int_{-r_{c}}^{0} \int_{\Omega_{s}}|z(t, \theta)|^{2} \mathrm{~d} x \mathrm{~d} \theta,
\end{aligned}
$$

for some constant $C>0$ independent on $\alpha$. Choosing $\alpha$ sufficiently small and then utilizing the equivalence of $\mathscr{L}$ and $\mathscr{E}$ yields the exponential stability of solutions with initial data in $H_{f} \times V_{s} \times L^{2}\left(\Omega_{s}\right) \times L^{2}\left(-r, 0 ; L^{2}\left(\Omega_{s}\right)\right)$.

\section{Appendix}

Given $T>0$ and $a<b$, we let $Q_{T}=(0, T) \times(a, b)$ and $(t, \theta)$ denote a typical point in $Q_{T}$. Define the operator $L=\partial_{t}-\partial_{\theta}$ and the consider the Hilbert space

$$
W_{L}^{2}\left(Q_{T}\right)=\left\{u \in L^{2}\left(Q_{T}\right): L u \in L^{2}\left(Q_{T}\right)\right\}
$$

equipped with the graph norm

$$
\|z\|_{W_{L}^{2}\left(Q_{T}\right)}=\left(\|z\|_{L^{2}\left(Q_{T}\right)}^{2}+\|L z\|_{L^{2}\left(Q_{T}\right)}^{2}\right)^{\frac{1}{2}} .
$$

Using mollifiers, one can see that $H^{1}\left(Q_{T}\right)$ is dense in $W_{L}^{2}\left(Q_{T}\right)$, for example see [2]. From [15, Proposition 18, Section 12], the set of all linear combinations $\chi_{A_{1}} u_{1}+\cdots+\chi_{A_{n}} u_{n}$, 
where $u_{i} \in H^{1}\left(Q_{T}\right)$ and $\chi_{A_{i}}$ is the indicator function of a measurable subset $A_{i}$ of $\Omega$, is dense in $L^{2}\left(\Omega ; W_{L}^{2}\left(Q_{T}\right)\right)$ endowed with the norm

$$
\|z\|_{L^{2}\left(\Omega ; W_{L}^{2}\left(Q_{T}\right)\right)}=\left(\|z\|_{L^{2}\left(\Omega ; L^{2}\left(Q_{T}\right)\right)}^{2}+\|L u\|_{L^{2}\left(\Omega ; L^{2}\left(Q_{T}\right)\right)}^{2}\right)^{\frac{1}{2}} .
$$

By mollifying these functions, we see that $C_{0}^{\infty}(\Omega) \otimes H^{1}\left(Q_{T}\right):=\operatorname{span}\left\{\phi \otimes u: \phi \in C_{0}^{\infty}(\Omega), u \in\right.$ $\left.H^{1}\left(Q_{T}\right)\right\}$ is dense also in $L^{2}\left(\Omega ; W_{L}^{2}\left(Q_{T}\right)\right)$.

Given $z \in L^{2}\left(\Omega ; W_{L}^{2}\left(Q_{T}\right)\right)$, define $\gamma_{g} z: L^{2}\left(\Omega ; W_{L}^{2}\left(Q_{T}\right)\right) \rightarrow \mathbb{R}$ according to

$$
\left(\gamma_{g} z\right)(\varphi)=\lim _{n \rightarrow \infty} \int_{\Omega} \int_{\partial Q_{T}} \chi_{\nu}\left(\gamma_{0} z_{n}\right) \cdot \phi \mathrm{d} s \mathrm{~d} x
$$

where $z_{n} \in C_{0}^{\infty}(\Omega) \otimes H^{1}\left(Q_{T}\right), z_{n} \rightarrow z$ in $L^{2}\left(\Omega ; W_{L}^{2}\left(Q_{T}\right)\right), \gamma_{0}: H^{1}\left(Q_{T}\right) \rightarrow H^{\frac{1}{2}}\left(\partial Q_{T}\right)$ is the trace operator and $\gamma_{0}(\phi \otimes u)=\phi \otimes \gamma_{0} u$ for $\phi \in C_{0}^{\infty}(\Omega)$ and $u \in H^{1}\left(Q_{T}\right)$. Also, $\chi_{\nu}=\chi_{\{\theta=b\} \cup\{t=T\}}-\chi_{\{\theta=a\} \cup\{t=0\}}$ where $\chi$ is the indicator function. It can be easily seen that the above limit exists and is independent of the sequence chosen to approximate $z$. Furthermore, we have $\gamma_{g} z \in L^{2}\left(\Omega ; H^{-\frac{1}{2}}\left(\partial Q_{T}\right)\right)$ and

$$
\gamma_{g} \in \mathcal{L}\left(L^{2}\left(\Omega ; W_{L}^{2}\left(Q_{T}\right)\right) ; L^{2}\left(\Omega ; H^{-\frac{1}{2}}\left(\partial Q_{T}\right)\right)\right)
$$

If $z \in L^{2}\left(\Omega ; H^{1}\left(Q_{T}\right)\right)$ then $\gamma_{g} z=\chi_{\nu} \gamma_{0} z$.

We now localize the traces which is useful in the transport equation (3.1). If $\Sigma \subset \partial Q_{T}$ then the space $\mathcal{V}(\Sigma)$ of all functions in $H^{\frac{1}{2}}\left(\partial Q_{T}\right)$ whose support is contained in $\Sigma$ is dense in $L^{2}(\Sigma)$, see $[44$, Theorem 13.6.10]. Denote by $V(\Sigma)$ the closure of $\mathcal{V}(\Sigma)$ with respect to the norm of $H^{\frac{1}{2}}\left(\partial Q_{T}\right)$.

Let $\Sigma^{0}=\{0\} \times(a, b), \Sigma^{T}=\{T\} \times(a, b), \Sigma_{a}=(0, T) \times\{a\}$ and $\Sigma_{b}=(0, T) \times\{b\}$ and we denote by $\Sigma$ to be either of these sets. Given $u \in L^{2}\left(\Omega ; W_{L}^{2}\left(Q_{T}\right)\right)$, define

$$
\left\langle\gamma_{\Sigma} z, \varphi\right\rangle_{L^{2}\left(\Omega ; V(\Sigma)^{\prime}\right) \times L^{2}(\Omega ; V(\Sigma))}=\lim _{n \rightarrow \infty} \int_{\Omega}\left\langle\gamma_{g} z, \chi_{\nu} \varphi_{n}\right\rangle_{H^{-\frac{1}{2}}\left(\partial Q_{T}\right) \times H^{\frac{1}{2}}\left(\partial Q_{T}\right)} \mathrm{d} x
$$

where $\varphi_{n} \in L^{2}(\Omega ; \mathcal{V}(\Sigma))$ and $\varphi_{n} \rightarrow \varphi$ in $L^{2}(\Omega ; V(\Sigma))$. It can be easily checked that

$$
\gamma_{\Sigma} \in \mathcal{L}\left(L^{2}\left(\Omega ; W_{L}^{2}\left(Q_{T}\right)\right) ; L^{2}\left(\Omega ; V(\Sigma)^{\prime}\right)\right) .
$$

Let $V_{L}^{2}\left(Q_{T}\right)$ be the completion of $H^{1}\left(Q_{T}\right)$ with respect to the norm

$$
\|z\|_{V_{L}^{2}\left(Q_{T}\right)}=\left(\|z\|_{W_{L}^{2}\left(Q_{T}\right)}^{2}+\left\|\gamma_{0} z\right\|_{L^{2}\left(\partial Q_{T}\right)}^{2}\right)^{\frac{1}{2}}
$$

For every $z \in L^{2}\left(\Omega ; V_{L}^{2}\left(Q_{T}\right)\right)$ it holds that $\gamma_{\Sigma} z \in L^{2}\left(\Omega ; L^{2}(\Sigma)\right)$. Indeed, suppose that $z_{n} \in L^{2}\left(\Omega ; H^{1}\left(Q_{T}\right)\right)$ and $z_{n} \rightarrow z$ in $L^{2}\left(\Omega ; V_{L}^{2}\left(Q_{T}\right)\right)$, and hence in $L^{2}\left(\Omega ; W_{L}^{2}\left(Q_{T}\right)\right)$. Thus we have $\gamma_{0} z_{n} \rightarrow \gamma_{0} z$ in $L^{2}\left(\Omega ; L^{2}\left(\partial Q_{T}\right)\right)$ and $\gamma_{\Sigma} z_{n} \rightarrow \gamma_{\Sigma} z$ in $L^{2}\left(\Omega ; V(\Sigma)^{\prime}\right)$. From the continuity of the embedding $L^{2}(\Sigma) \subset V(\Sigma)^{\prime}$, it follows that $\left.\gamma_{0} z\right|_{\Sigma}=\gamma_{\Sigma} z$. In particular, $\gamma_{g} z \in L^{2}\left(\Omega ; L^{2}\left(\partial Q_{T}\right)\right)$ for every $z \in L^{2}\left(\Omega ; V_{L}^{2}\left(Q_{T}\right)\right)$.

With these preliminaries, it follows by a density argument that the following integration by parts formula

$$
\int_{\Omega} \int_{0}^{T} \int_{a}^{b} \varphi \cdot L^{*} \psi \mathrm{d} \theta \mathrm{d} t \mathrm{~d} x-\int_{\Omega} \int_{0}^{T} \int_{a}^{b} L \varphi \cdot \psi \mathrm{d} \theta \mathrm{d} t \mathrm{~d} x=\int_{\Omega} \int_{\partial Q_{T}} \chi_{\nu} \gamma_{g} \psi \cdot \gamma_{g} \varphi \mathrm{d} s \mathrm{~d} x
$$

where $L^{*}=-\partial_{t}+\partial_{\theta}$, holds for every $\varphi, \psi \in L^{2}\left(\Omega ; V_{L}^{2}\left(Q_{T}\right)\right)$. 


\section{REFERENCES}

[1] S. Agmon, A. Douglis, and L. Nirenberg, Estimates near the boundary for solutions of elliptic partial differential equations satisfying general boundary value conditions, I, Comm. Pure Appl. Math. 12, pp. 623-727, (1959)

[2] N. Antonić and K. Burazin, Graph spaces of first-order linear partial differential operators, Math. Commun. 14, pp. 135-155, (2009)

[3] G. Avalos and F. Bucci, Rational rates of uniform decay for strong solutions to a fluid-structure PDE system, J. Differential Equations 258, pp. 4398-4423 (2015)

[4] G. Avalos and R. Triggiani, The coupled PDE system arising in fluid-structure interaction, Part I: Explicit semigroup generator and its spectral properties, Contemporary Mathematics 440, pp. 15-54, (2007)

[5] G. Avalos and R. Triggiani, Semigroup wellposedness in the energy space of a parabolic-hyperbolic coupled Stokes-Lamé PDE system of fluid-structure interaction, Discr. Cont. Dynam. Sys. 2, pp. 417447, (2009)

[6] G. Avalos and R. Triggiani, Rational decay rates for a PDE heat-structure interaction: a frequency domain approach, Evol. Equ. Control Theory 2, pp. 233-253, (2013)

[7] G. Avalos and R. Triggiani, Fluid structure interaction with and without internal dissipation of the structure : A contrast study in stability, Evol. Equ. Control Theory 2, pp. 563-598, (2013)

[8] G. Avalos, I. Lasiecka and R. Trigianni, Higher regularity of a coupled parabolic-hyperbolic fluid structure interactive system, Georgian Math. J. 15, 403-437, (2008)

[9] V. Barbu, Z. Grujić, I. Lasiecka and A. Tuffaha, Existence of the energy-level weak solutions for a nonlinear fluid-structure interaction model, Contemporary Mathematics 440, pp. 55-81, (2007)

[10] V. Barbu, Z. Grujić, I. Lasiecka and A. Tuffaha, Smoothness of weak solutions to a nonlinear fluidstructure interaction model, Indiana U. Math. J. 57, pp. 1173-1207, (2008)

[11] H. Beiaro Da Veiga, On the existence of strong solutions to a coupled fluid-structure evolution problem, Journal of Mathematical Fluid Mechanics 6, pp. 21-52, (2004)

[12] D. Coutand and S. Shkoller, Motion of an elastic solid inside an incompressible viscous fluid, Arch. Ration. Mech. Anal. 176, pp. 25-102, (2005)

[13] R. Datko, Not all feedback stabilized hyperbolic systems are robust with respect to small time delays in their feedback, SIAM J. Control Optim. 26, pp. 697-713, (1988)

[14] R. Datko, J. Lagnese and P. Polis, An example on the effect of time delays in boundary feedback stabilization of wave equations, SIAM J. Control Optim 24, pp. 152-156, (1985)

[15] N. Dinculeanu, Vector Measures, Pergamon Press, (1967)

[16] Q. Du, M. D. Gunzburger, L. S. Hou, and J. Lee, Analysis of a linear fluid-structure interaction problem, Discr. and Contin. Dynam. Systems 9, pp. 633-650, (2003)

[17] K. O. Friedrichs, Symmetric positive linear differential equations, Comm. Pure Appl. 11, pp. 333-418, (1958)

[18] L. Hörmander, The Analysis of Linear Partial Differential Operators III: Pseudo-differential operators, Springer-Verlag, Heidelberg, (1985)

[19] M. A. Horn, Sharp trace regularity for the solutions of the equations of dynamic elasticity, Journal of Mathematical Systems, Estimation and Control 8, pp. 217-219, (1998)

[20] M. Ignatova, I. Kukavica, I. Lasiecka and A. Tuffaha, On well-posedness and small data global existence for an interface damped free boundary fluid-structure model, Nonlinearity 27, pp. 467-499 (2014)

[21] M. Ignatova, I. Kukavica, I. Lasiecka and A. Tuffaha, Small data global existence for a fluid-structure model, Nonlinearity 30, pp. 848-898, (2017)

[22] M. Jensen, Discontinuous Galerkin methods for Friedrichs systems with irregular solutions. PhD thesis, University of Oxford, (2004)

[23] M. Kirane and B. Said-Houari, Existence and asymptotic stability of a viscoelastic wave equation with delay, Z. Angew. Math. Phys. 62, pp. 1065-1082, (2011)

[24] I. Kukavica and A. Tuffaha, Wel-posedness for a compressible Navier-Stokes-Lamé system with a free interface, Nonlinearity 25, pp. 3111-3137, (2012)

[25] I. Kukavica, A. Tuffaha and M. Ziane, Strong solutions to a Navier-Stokes-Lamé system in a domain with non-flat boundary, Nonlinearity 24, pp. 159-176, (2011)

[26] I. Lasiecka and Y. Lu, Asymptotic stability of finite energy in Navier Stokes-elastic wave interaction, Semigroup Forum 82, pp. 61-82, (2011)

[27] I. Lasiecka and Y. Lu, Interface feedback control stabilization of a nonlinear fluid-structure interaction, Nonlinear Analysis 75, pp. 1449-1460, (2012) 
[28] J. L. Lions, Quélques Méthods de Résolution des Problémes aux Limits Nonlinéaries, Dunod, Paris, (1969).

[29] J. L. Lions and E. Magenes, Non-homogeneous Boundary Value Problems and Applications, Vol. 1, Springer-Verlag, New York, (1972)

[30] Y. Lu, Stabilization of a fluid structure interaction with nonlinear damping, Control and Cybernetics 42 , pp. 155-181, (2013)

[31] Y. Lu, Uniform decay rates for the energy in nonlinear fluid structure interaction with monotone viscous damping, Palest. J. Math. 2, pp. 215-232, (2013)

[32] R. B. Melrose, Microlocal parametrices for diffractive boundary value problems, Duke Math. J. 42, pp. 605-635, (1975)

[33] R. B. Melrose and J. Sjöstrand, Singularities of boundary value problems, I. Comm. Pure Appl. Math. 31, pp. 593-617, (1978)

[34] M. Mitrea, S. Monniaux and M. Wright, The Stokes operator with Neumann boundary conditions in Lipschitz domains, Journal of Mathematical Sciences 176, pp. 409-457, (2011)

[35] J. Nečas, Les méthodes directes en théorie des équations elliptiques. Academia, Editions de l' Acad. Tchécoslovaque des Sciences, Prague (1967).

[36] S. Nicaise and C. Pignotti, Stability and instability results of the wave equation with a delay term in boundary or internal feedbacks, SIAM J. Control Optim. 45, pp. 1561-1585, (2006)

[37] S. Nicaise and C. Pignotti, Stabilization of the wave equation with boundary or internal distributed delay, Differential and Integral Equations 21, pp. 935-958, (2008)

[38] G. Peralta, A fluid-structure interaction model with interior damping and delay in the structure, Zeitschrift fuer angewandte Mathematik und Physik 67, pp. 1-20, (2016)

[39] G. Peralta and G. Propst, Well-posedness and regularity of linear hyperbolic systems with dynamic boundary conditions, Proc. Roy. Soc. Edin. Section A: Mathematics 156, pp. 1047-1080, (2016)

[40] J. Simon, Compact sets in the space $L^{p}(0, T ; B)$, Annali di Matematica pura ed applicata (IV), Vol. CXLVI, pp. 65-96, (1987)

[41] H. Sohr, The Navier-Stokes Equations: An Elementary Functional Analytic Approach, Birkhäuser, Berlin, (2001)

[42] J. Sokolowski and J.-P. Zolesio, Introduction to Shape Optimization: Shape Sensitivity Analysis, Springer-Verlag, Heidelberg, (1992)

[43] R. Temam, Navier-Stokes Equations, Theory and Numerical Analysis, AMS Chelsea Publishing, Providence, Rhode Island, (2001)

[44] M. Tucsnak and G. Weiss, Observation and Control for Operator Semigroups, Birkhäuser-Verlag, Basel, (2009)

[45] J. Zhang, The analyticity and exponential decay of a Stokes-wave coupling system with viscoelastic damming in the variational framework, Evol. Equ. Control Theory 6, pp. 135-154, (2017) 